Pacific Journal of Mathematics

CALCULATION OF AXIALLY SYMMETRIC CAVITIES AND
JETS 


\title{
CALCULATION OF AXIALLY SYMMETRIC CAVITIES AND JETS
}

\author{
P. R. Garabedian
}

1. Introduction. We shall study in this paper steady, axially symmetric, irrotational flows of an incompressible liquid. We shall be interested in motions that exhibit a free surface along which the liquid is bounded by a gas whose inertia is negligible relative to that of the liquid, so that the gas can be assumed to be at rest and to have constant pressure. The determination of flows of this type is a mathematical problem of exceptional difficulty because the shape of the free surface is not known and must be calculated as part of the solution. In the case of axial symmetry, no systematic method has as yet been developed for finding free surface flows past prescribed obstacles, although a few calculations, notably those by Trefftz [17] and by Southwell and Vaisey [16], have been executed on a basis of inspired guesswork. The chief drawback of the work done by these investigators is that their successive approximations to the shape of the free surface are obtained by trial and error and are slow to converge.

Our hope in the present article is to present techniques for the systematic calculation of free surface flows, with emphasis on the axially symmetric case. Recent advances $[3,4,5,6,7,18]$ in the mathematical theory of cavitational flow form the basis for our method. Although rigorous proof of the convergence of the series expansion and of the iterative scheme which we shall use appears to be too difficult to undertake at this time, nevertheless sound theoretical reasons are given for expecting the procedures to converge. This is in contrast with other schemes that the author has seen suggested, such as the interation process for the Trefftz integral equation, which theory would predict to diverge for more or less the same reason that the classical Neumann series for solution of the Dirichlet problem diverges, namely, because the lowest relevant eigenvalue does not exceed 1 . The difference here between our approach and the earlier ones is analogous to the difference between solving an equation $x=f(x)$ by Newton's method, for which we expect rapid convergence, and solving the same equation by successive approximations of the type $x_{n+1}=f\left(x_{n}\right)$, which will diverge if the derivative of the function $f(x)$ exceeds 1 . The significant disagreement that will be found between our numerical results and the earlier work in the field can be attributed partly to this failure of convergence and partly to the insensitive nature of the guesswork involved in the other methods.

Received December 6, 1955 , 
We shall confine ourselves in this paper to only the simplest models of axially symmetric free surface flow, since treatment of more general problems does not add difficulties in principle, but does lead to tedious and inscrutable calculations. Our first study will be concerned with the vena contracta; we calculate the contraction coefficient of a jet issuing from a circular orifice in a plane wall. Our next model will be the infinite cavity behind a circular disk, for which we compute the drag coefficient. Our final and most difficult model will be the finite Riabouchinsky cavity between two circular disks. In this case we prescribe the cavity length and must calculate not only the drag coefficient, but also the cavitation parameter and the cavity width. By carrying through the computations both for a finite and for an infinite cavity, we are able to present a theoretical plot of the drag coefficient as a function of the cavitation parameter.

In order to derive the numerical results, it is necessary for us to extend considerably the mathematical theory of cavities and jets. This material is quite possibly as interesting and informative as the numerical work itself. The plan of the paper will be to discuss theory and qualitative analysis in the early sections and to proceed to numerical examples in the later sections.

At this point we summarize our theoretical contributions and outline our method of attack. The Stokes stream function $\psi$ of an axially symmetric flow satisfies in the meridian plane the partial differential equation

$$
\Delta \psi-\frac{1}{y} \psi_{y}=\psi_{x x}+\psi_{y y}-\frac{1}{y} \psi_{x}=0
$$

where $x$ is the coordinate measured along the axis of symmetry and $y$ represents the distance from this axis. We consider the free boundary problem for the more general equation

$$
\Delta \psi-\frac{\varepsilon}{y} \psi_{y}=0
$$

and study the dependence of the solution on the parameter $\varepsilon$. For $\varepsilon=$ 0 , equation (1.2) governs the stream function of a plane flow. Since a variety of plane flows with free boundaries are known explicitly, we are able to develop the stream function $\psi$ of the corresponding free boundary problems for (1.2) in perturbation series in powers of $\varepsilon$. We interpolate to estimate quite accurately the desired solution of the threedimensional problem (1.1) corresponding to $\varepsilon=1$ by combining the information obtained from the series development with an analysis of the degenerate cases $\varepsilon=-1$ and $\varepsilon=+\infty$. 
In applying the perturbation method to the free boundary conditions we are led to a linear mixed boundary value problem for the first order term. A generalization of this mixed problem yields a systematic determination of the corrections required for a scheme of successive approximations to the free boundary. The difference between the stream functions of two consecutive approximations satisfies here the linear mixed boundary value problem in question. When the initial terms of the perturbation series do not provide sufficiently accurate results, we resort to successive applications of the mixed problem to improve our approximations at an exponential rate of convergence comparable to that obtained in Newton's method.

The actual execution of the perturbation method is significantly eased by a reformulation of the free boundary problem for (1.2) in terms of an analytic function $g(z)$ of the complex variable $z=x+i y$. This reformulation is based on an earlier treatment of axially symmetric free surface flows [4], and it leads to interesting qualitative results. In particular, we establish in this way that, for all our models, the free boundary depends monotonically on the parameter $\varepsilon$.

Various other auxiliary theorems are derived in the paper which are useful in the numerical calculation of cavities and jets. For small values of the cavitation parameter we establish asymptotic formulas for the drag and cavity dimensions in the case of the Riabouchinsky model. Some of these formulas confirm theoretically information which has heretofore been deduced on an empirical basis for axially symmetric flows [14]. Among the relationships which we derive for the gross physical quantities describing cavity flow is the formula

$$
4 h D=3(\sigma V-M)
$$

expressing the drag $D$ in terms of the cavity length $2 h$, the cavitation parameter $\sigma$, the cavity volume $V$ and the virtual mass $M$. The combination of $\sigma, V$ and $M$ appearing on the right in (1.3) has been shown $[5,6]$ to be a maximum for free surface flows. Thus, although this formula is valid only for conical nose shapes, it is nevertheless significant, because the stationary character of the quantities involved permits us to estimate the drag with an accuracy proportional to the square of the error in our approximation to the cavity shape.

The arguments and proofs presented in this paper will in some cases meet the standards of full mathematical rigor and in others will depend more on heuristic reasoning. No further comments will be made in this connection, since the mathematicians interested in more precise formulation will easily discern where there are problems still calling for study, while the readers whose main interest is in the physical implications of the investigation should find all the material on a sound theoretical basis 
and the arguments thoroughly convincing and substantial.

2. Variational expression for the drag. In this section we discuss three-dimensional free surface flows which do not necessarily possess axial symmetry. We shall consider flows that are uniform at infinity with speed 1 , and we shall divide the boundary $\Gamma$ of the fluid into two parts, the fixed boundary $\Gamma_{1}$ and the free boundary $\Gamma_{2}$. The flow is governed by a velocity potential $\varphi$ which is harmonic in the region $\Omega$ exterior to the surface $\Gamma$ and which has an expansion of the form

$$
\varphi=x+\frac{a x}{r^{3}}+\cdots
$$

in the neighborhood of infinity, where $x$ is the rectangular coordinate measured in the direction of the motion at infinity, where $r$ is the distance from the origin, and where $a$ is a constant whose physical significance will be discussed presently. We assume that the density of the fluid is 1, and thus Bernoulli's law gives for the pressure $p$ the equation

$$
\frac{1}{2}(\nabla \varphi)^{2}+p=\text { const }
$$

The velocity potential $\varphi$ has a vanishing normal derivative

$$
\frac{\partial \varphi}{\partial n}=0
$$

along the complete boundary $\Gamma$, and in addition the pressure $p$ is constant along the free surface $\Gamma_{2}$, so that by $(2.2)$ we have there a second boundary condition of the form

$$
(\nabla \varphi)^{2}=1+\sigma,
$$

where $\sigma$ is the cavitation parameter of the flow.

We interpret the fixed boundary $\Gamma_{1}$ as a system of rigid shells, whereas the remainder of the region inside the boundary surface $\Gamma$ will be considered to contain vapor, or gas, whose total volume we denote by $V$. The virtual mass $M$ of our fluid motion is simply the energy integral

$$
M=\iiint_{\Omega}(\nabla \varphi-\nabla x)^{2} d x d y d z
$$

extended over the entire flow region $\Omega$. The volume $V$ and the virtual mass $M$ are related to the coefficient $a$ occurring in the expansion (2.1) by the familiar formula [6] 


$$
4 \pi a=M+V .
$$

For the physical problems arising in the study of cavitation, the rigid walls $\Gamma_{1}$ are known, but the free surface $\Gamma_{2}$ must be determined, presumably from the extra boundary condition (2.4). However, it has been shown $[5,6]$ that if we drop the auxiliary condition (2.4) and allow the free surface $\Gamma_{2}$ to vary while $\Gamma_{1}$ is held fixed, the choice of $\Gamma_{2}$ which solves the extremal problem

$$
M-\sigma V=4 \pi a-(1+\sigma) V=\text { minimum }
$$

selects $\Gamma_{2}$ as the free surface satisfying the constant pressure condition (2.4). This result follows from the fact that if we subject the variable surface $\Gamma_{2}$ to an infinitesimal normal displacement of magnitude $\delta n$, the consequent first order perturbations $\delta V, \delta a$ and $\delta M$ in the physical quantities $V, a$ and $M$ are given by the Hadamard formulas [6]

$$
\begin{gathered}
\delta V=\iint_{\Gamma_{2}} \delta n d A, \\
\delta a=\frac{1}{4 \pi} \iint_{\Gamma_{2}}(\nabla \varphi)^{2} \delta n d A, \\
\delta M=\iint_{\Gamma_{2}}\left[(\nabla \varphi)^{2}-1\right] \delta n d A,
\end{gathered}
$$

where $d A$ is the area element along the surface $\Gamma_{2}$. From these formulas we verify, indeed, that (2.4) is equivalent to the variational statement that

$$
\delta M-\sigma \delta V=0
$$

for every possible choice of the displacement $\delta n$.

The variational principle (2.7) would obviously be suitable for estimating the energy of cavitating flows. We shall establish that for special shapes of the fixed boundary $\Gamma_{1}$ the variational principle can also be used to calculate the drag. We suppose henceforth that $\Gamma_{1}$ consists of two portions, each situated opposite to the other on a facing pair of symmetrically placed circular cones whose axes coincide with the $x$-axis and whose vertices lie $h$ units from the origin. A case of particular interest occurs when the two portions of $I_{1}$ consist of symmetric sections of planes perpendicular to the $x$-axis. The free surface $\Gamma_{2}$ is a sheath connecting the two parts of the rigid boundary $\Gamma_{1}$, and the flow is merely a generalization of the finite cavity model due to Riabouchinsky.

Let us now magnify the whole configuration $\Gamma$ by a factor $1+\eta$, where $\eta$ is a small positive number. There will result from this infini- 
tesimal magnification an infinitesimal normal displacement $\delta n$ of the surface $\Gamma$, and thus formulas (2.8) and (2.10) yield the relation

$$
\delta M-\sigma \delta V=\iint_{\Gamma}\left[(\nabla \varphi)^{2}-1-\sigma\right] \delta n d A
$$

for the corresponding increments in $M$ and $V$. Along the free surface $\Gamma_{2}$ the integrand in (2.12) vanishes, according to (2.4), while on $\Gamma_{1}$ we find easily that

$$
\delta n=\eta h \sin \theta,
$$

where $\theta$ is the half-angle of the cones forming $\Gamma_{1}$. Therefore, by (2.12),

$$
\begin{gathered}
\delta M-\sigma \delta V=\iint_{\Gamma_{1}}\left[(\nabla \varphi)^{2}-1-\sigma\right] \eta h \sin \theta d A \\
=\eta h \iint_{\Gamma_{1}}\left[(\nabla \varphi)^{2}-1-\sigma\right] d y d z .
\end{gathered}
$$

The integral on the right consists of two parts evaluated over the two opposite portions of $\Gamma_{1}$. One of these two portions of $\Gamma_{1}$ is considered to be the actual obstacle in the flow, and the $\operatorname{drag} D$ due to fluid pressure on this obstacle is given according to Bernoulli's law (2.2) by the integral

$$
D=\frac{1}{2} \iint\left[1+\sigma-(\nabla \varphi)^{2}\right] d y d z
$$

extended over this portion of $\Gamma_{1}$. By d'Alembert's paradox, the force exerted on the remaining portion of $\Gamma_{1}$ must be equal and opposite to the drag $D$, and thus the two parts of the integral (2.14) over $\Gamma_{1}$ are equal and each has the value $-2 D$. Hence (2.14) states that

$$
\delta M-\sigma \delta V=-4 \eta h D
$$

On the other hand, $V$ and $M$ have the dimension of length cubed, so that they actually increase by the factor $(1+\eta)^{3}$. Since $\sigma$ is dimensionless, this yields directly the relation

$$
\delta M-\sigma \delta V=3 \eta(M-\sigma V) .
$$

Comparing equations (2.16) and (2.17) and dividing out the infinitesimal factor $\eta$, we obtain the fundamental expression

$$
4 h D=3(\sigma V-M)
$$

for the drag $D$ in terms of the virtual mass $M$. 
The significance of formula (2.18) for the calculation of the drag $D$ lies in the stationary character of the terms on the right under small shifts of the free surface $\Gamma_{2}$. If we have made quite accurate estimates of the cavitation parameter $\sigma$ and the cavity length $2 h$, while our error in determining $\Gamma_{2}$ is of the order $\delta n$, then we can calculate $D$ with an error of the order of magnitude $(\delta n)^{2}$ by substituting into (2.18) values of $V$ and $M$ computed accurately for the flow past our approximation to $\Gamma$. Because of the minimum principle (2.7) we can even state that the error occurring in this calculation of $D$ is negative. In other words, our variational principle gives an exceptionally sharp lower bound for the drag $D$.

An interesting qualitative analysis of three-dimensional cavitational flows without axial symmetry can be based on formula (2.18) and the variational identity (2.11). For the sake of simplicity, let us assume that the two portions of the fixed boundary $\Gamma_{1}$ lie in parallel planes and are symmetric with respect to the $y$-axis and $z$-axis. In particular, they might be elliptical plates. We hold $\sigma$ fixed and continuously alter the shape of the plates $\Gamma_{1}$ in such a manner that $h$ remains invariant. For the case of elliptical plates, this implies that for each choice of the eccentricity the size of the plates is uniquely determined. The infinitesimal normal displacement $\delta n$ of the liquid surface $\Gamma$ generated by this continuous process must vanish on the rigid boundary $\Gamma_{1}$ because of the invariance of $h$. Hence the relation (2.11) is valid, and it follows from (2.18) that the drag $D$ remains unchanged. Thus for a flat plates of variable shape the drag $D$ depends only on the cavitation parameter $\sigma$ and the cavity length $2 h$. Therefore it suffices to calculate $D$ for the axially symmetric case of a circular disk alone in order to obtain $D$ directly for such plate shapes as the ellipse or the rectangle. It would be interesting to have experimental confirmation of this invariance phenomenon.

The invariance of $D$ casts some doubt on the existence of threedimensional cavity flows for the Riabouchinsky model with a flat nose which is quite long and narrow. Indeed, with $D$ invariant the limit of the three-dimensional cavity flow past an elliptical plate whose eccentricity approaches 1 cannot be the plane flow past a vertical segment, since if the limit process could be performed smoothly, it would be the drag coefficient, and not the drag itself, that would remain finite. One is left to deduce that the flow degenerates in some unknown manner. Another limiting case to substantiate this deduction occurs when the plane nose consists of two fixed circular disks joined by an ever-narrowing strut. If the cavity behind such a nose were to remain well-defined, it is difficult to imagine what the limiting flow could be when the strut degenerates completely. The nature of the curve of separation may be 
at the root of this anomaly.

3. Dependence on the dimension parameter. We turn our attention to generalized axially symmetric free surface flows in space of $\varepsilon+2$ dimensions. The velocity potential $\varphi$ and the stream function $\psi$ for such flows obey the elliptic system of partial differential equations

$$
\varphi_{x}=\frac{1}{y^{\varepsilon}} \psi_{y}, \quad \varphi_{y}=-\frac{1}{y^{\varepsilon}} \psi_{x}
$$

in the meridian plane of the coordinates $x$ and $y$, where $x$ measures distance along the axis of symmetry and $y$ measures the perpendicular distance from this axis. We shall denote the meridian cross section of the flow region by $\Omega$ and we shall denote its boundary by $\Gamma$. Since $\Gamma$ is a streamline, we always require $\psi=0$ there. We divide $\Gamma$ again into fixed boundary $\operatorname{arcs} \Gamma_{1}$ and a free boundary $\Gamma_{2}$. Along $\Gamma_{2}$ the generalized constant pressure condition

$$
\begin{array}{cl}
1 & \partial \psi \\
y^{\varepsilon} & \partial n
\end{array}
$$

is imposed. We can eliminate $\varphi$ from (3.1) to obtain for $\psi$ the single second order equation

$$
\psi_{x x}+\psi_{y y}-\frac{\varepsilon}{y} \psi_{y}=0,
$$

and we shall be interested in using the dependence of the solution $\psi$ on the parameter $\varepsilon$ as a tool for investigating the physically significant case $\varepsilon=1$ of genuine axially symmetric flow in three-dimensional space.

We start by convincing ourselves that for a properly set free boundary problem $\psi$ should be a regular analytic function of $\varepsilon$ for all complex values of $\varepsilon$ lying in the half-plane

$$
\mathscr{R}\{\varepsilon\}>-1 \text {. }
$$

This conclusion is based on a consideration of the Dirichlet integral

$$
\iint\left(\psi_{x}^{2}+\psi_{y}^{2}\right) \frac{d x d y}{y^{\varepsilon}}=\iint\left(\varphi_{x}^{2}+\varphi_{y}^{2}\right) y^{\varepsilon} d x d y
$$

whose Euler equation is (3.3). The established theorem on the existence of axially symmetric free surface flows [5] is based on the minimum problem (2.7) for the virtual mass $M$ and thus involves a Dirichlet integral of the type (3.5). Since the Dirichlet integral (3.5) makes sense for a flow region including the axis of symmetry only under the restriction (3.4), we are able to formulate the existence proof only for such 
values of $\varepsilon$, and even for these values the problem poses numerous difficulties. Nevertheless, we expect a unique solution $\psi$ to exist for each $\varepsilon$ in the half-plane (3.4) and hence we expect $\psi$ to be regular there in its dependence on $\varepsilon$.

Our convictions in this respect are substantiated by knowledge of the explicit formula

$$
\psi=\frac{y^{1+\varepsilon}}{1+\varepsilon}-\frac{y^{1+\varepsilon}}{(1+\varepsilon) r^{2+\varepsilon}}, \quad r^{2}=x^{2}+y^{2},
$$

for the flow past the unit sphere $r=1$, which exhibits a singularity only when $\varepsilon=-1$. While for every $\varepsilon>-1$ the stream function $\psi$ defined by (3.6) describes uniform flow at infinity, the limit of $(1+\varepsilon) \psi$ as $\varepsilon \rightarrow-1$ has spherical level surfaces and is best interpreted physically as the Green's function of a three-dimensional sphere.

Although the existence proof used above to establish regularity of $\psi$ in the half-plane (3.4) has been worked out in detail only for cavities [5], it can be carried through equally well for jets, and hence the regularity should be as described for all the models we shall have occasion to treat. The significance of the region of regularity in the $\varepsilon$-plane is that it indicates the radius of convergence of the series expansion

$$
\psi(x, y ; \varepsilon)=\psi_{0}(x, y)+\varepsilon \psi_{1}(x, y)+\varepsilon^{2} \psi_{2}(x, y)+\cdots
$$

of $\psi$ in powers of $\varepsilon$, which will be the principal item under discussion in this section. Since (3.4) is equivalent to the inequality

$$
\left|\frac{\varepsilon}{\varepsilon+2}\right|<1,
$$

we can produce convergence in the entire region of established regularity by introducing the substitution

$$
\delta=\frac{\varepsilon}{\varepsilon+2}
$$

and rearranging the series (3.7) in increasing powers of the new variable $\delta$. This brings the relally relevant value $\varepsilon=1$, corresponding to $\delta=1 / 3$, well inside the circle of convergence $|\delta|<1$.

The form of equation (3.3) suggests that it should not be too difficult to determine in succession for a prescribed free boundary problem the coefficients $\psi_{0}, \psi_{1}, \psi_{2}, \cdots$ of the series expansion (3.7). However, since $\psi$ behaves like $y^{1+\varepsilon}$ for small values of $y$, it turns out to be more convenient to introduce the new unknown function

$$
U=\frac{\psi}{y^{\varepsilon}}
$$


and to study instead its power series development

$$
U(x, y ; \varepsilon)=U_{0}(x, y)+\varepsilon U_{1}(x, y)+\varepsilon^{2} U_{2}(x, y)+\cdots .
$$

The first term $U_{0}$ is simply the stream function for the plane free boundary problem, and it can be determined explicitly by the hodograph method whenever the fixed boundary $\Gamma_{1}$ consists of polygonal arcs. By substituting the power series (3.11) into the differential equation

$$
U_{x x}+U_{y y}+\frac{\varepsilon}{y} U_{y}-\frac{\varepsilon}{y^{2}} U=0
$$

satisfied, according to (3.3), by $U$, and by equating to zero the factors multiplying the various powers of $\varepsilon$ in the resulting identity, we obtain the recursive system of Poisson equations

$$
\Delta U_{j}=\frac{1}{y^{2}} U_{j-1}-\frac{1}{y} \frac{\partial U_{1-1}}{\partial y}
$$

for the higher coefficients $U_{1}, U_{2}, \ldots$. All the terms $U_{j}$ satisfy on the fixed boundary $\Gamma_{1}$ the same boundary condition

$$
U,=0,
$$

and by deriving a further recursive system of boundary conditions for the $U_{j}$ on the free boundary $\Gamma_{2}$ of the known flow region $\Omega$ for the plane case $\varepsilon=0$, we shall contrive to formulate a set of linear boundary value problems in this known region $\Omega$ which determine systematically and uniquely all the coefficients $U_{j}$.

For arbitrary values of $\varepsilon$ we represent the free boundary curve $\Gamma_{2}$ in the parametric form

$$
z=z(s ; \varepsilon)
$$

in terms of the arc length $s$ measured, with the flow region on the left, from the point of separation from $\Gamma_{1}$. We introduce the notations

$$
\dot{z}=\frac{\partial z}{\partial s}, \quad \frac{\partial}{\partial z}=\frac{1}{2}\left(\frac{\partial}{\partial x}-i \frac{\partial}{\partial y}\right), \quad-\frac{\partial}{\partial \bar{z}}=\frac{1}{2}\left(\frac{\partial}{\partial x}+i \frac{\partial}{\partial y}\right) .
$$

According to the definition (3.10), we must have $U=0$ on $I_{2}^{\prime}$, and combining this with the constant pressure condition (3.2), we obtain the single complex free boundary condition

$$
\varphi+i U=s
$$

along $\Gamma_{2}$, by virtue of the differential system (3.1), which can now be expressed in the form 


$$
\varphi_{\bar{z}}+i U_{\bar{z}}=\frac{\varepsilon U}{2 y} .
$$

Because of (3.18) and the relation $U=0$ on $\Gamma_{2}$, differentiation of (3.17) with respect to $s$ yields

$$
\varphi_{z}+i U_{z}=\overline{\dot{z}}
$$

We note that since $s$ represents arc length, we have

$$
\dot{z} \overline{\dot{z}}=1, \quad \dot{z} \frac{\partial \overline{\dot{z}}}{\partial \varepsilon}+\overline{\dot{z}} \frac{\partial \dot{z}}{\partial \varepsilon}=2 \mathscr{R}\left\{\overline{\dot{z}} \frac{\partial \dot{z}}{\partial \varepsilon}\right\}=0, \quad \ddot{z}=-i \kappa \dot{z}
$$

along $\Gamma_{2}$, where $\kappa$ denotes the curvature.

With these preliminaries in mind, we proceed to differentiate the free boundary condition (3.17) with respect to $\varepsilon$. We find

$$
\varphi_{\varepsilon}+i U_{\varepsilon}+\left(\varphi_{z}+i U_{z}\right) \frac{\partial z}{\partial \varepsilon}+\left(\varphi_{\bar{z}}+i U_{\bar{z}}\right) \frac{\partial \bar{z}}{\partial \varepsilon}=0,
$$

whence by (3.18) and (3.19)

$$
\left(\varphi_{\varepsilon}+i U_{\varepsilon}\right) \dot{z}+\frac{\partial z}{\partial \varepsilon}=0,
$$

since $U=0$ on $\Gamma_{2}$. Differentiating (3.22) with respect to $s$ and multiplying by $\overline{\dot{z}}$, we obtain

$$
-i \kappa\left(\varphi_{\varepsilon}+i U_{\varepsilon}\right)+\frac{\partial \varphi_{\varepsilon}}{\partial s}+i \frac{\partial U_{\varepsilon}}{\partial s}+\overline{\dot{z}} \frac{\partial \dot{z}}{\partial \varepsilon}=0
$$

We take the real part of (3.23) to derive by (3.20) the relation

$$
\frac{\partial \varphi_{\varepsilon}}{\partial s}+\kappa U_{\varepsilon}=0
$$

On the other hand, differentiation of (3.18) with respect to $\varepsilon$ yields

$$
\frac{\partial \varphi_{\varepsilon}}{\partial \bar{z}}+i \frac{\partial U_{\varepsilon}}{\partial \bar{z}}=\frac{\varepsilon U_{\varepsilon}}{2 y}+\frac{U}{2 y},
$$

so that we can eliminate $\varphi_{\varepsilon}$ from (3.24) to obtain along the free boundary $\Gamma_{2}$ the condition

$$
\frac{\partial}{\partial n}\left(y^{\varepsilon} U_{\varepsilon}\right)+\kappa y^{\varepsilon} U_{\varepsilon}=0
$$

for the function $U_{\varepsilon}$ alone.

If we set $\varepsilon=0$ in (3.26), we arrive at the boundary condition 


$$
\frac{\partial U_{1}}{\partial n}+\kappa U_{1}=0
$$

for the first order coefficient $U_{1}$. By differentiating (3.26) repeatedly with respect to $\varepsilon$ and setting $\varepsilon=0$, we can derive analogous boundary conditions along $\Gamma_{2}$ for the higher coefficients $U_{j}$. All these conditions have the form

$$
\frac{\partial U_{j}}{\partial n}+\kappa U_{j}=B_{j}
$$

where the $B_{j}$ are known expressions involving only the earlier coefficients $U_{0}, \cdots, U_{j-1}$. Thus we can find in succession all the coefficients $U_{j}$ by solving, one after the other, the linear mixed boundary value problems (3.13), (3.14), (3.28) in the known region $\Omega$ of the plane flow calculated for $\varepsilon=0$. For larger values of $j$ this program turns out to be unfeasible in practice, but we show in this paper how even the first two terms $U_{0}$ and $U_{1}$ can be used to give a good approximation for three-dimensional axially symmetric free surface flows.

We indicate here how to solve the mixed boundary value problem (3.13), (3.14), (3.28) in closed form when $\Gamma_{1}$ consists of polygonal arcs. It suffices to determine in the plane flow region $\Omega$ a Green's function $H\left(z_{1}, z_{2}\right)$ with the following properties. At the point $z_{1}=z_{2}$, the function $H$ has a logarithmic singularity of the form

$$
H\left(z_{1}, z_{2}\right)=\log \frac{1}{\left|z_{1}-z_{2}\right|}+\cdots,
$$

while in the rest of $\Omega$ it remains bounded and satisfies Laplace's equation

$$
\Delta H=0 \text {. }
$$

The boundary condition

$$
H=0
$$

is imposed on $\Gamma_{1}$, whereas along $\Gamma_{2}$ we require

$$
\frac{\partial H}{\partial n}+\kappa H=0 \text {. }
$$

The solutions of (3.13), (3.14) and (3.28) can easily be expressed in terms of $H$ and the given data by means of Green's formula.

We denote by

$$
\zeta=\varphi_{0}+i \varphi_{0}
$$


the complex potential of our plane free streamline flow, and we consider the conformal transformation

$$
w=\frac{1}{i} \log \left(i \frac{d \zeta}{d z}\right)
$$

of the flow region $\Omega$ onto a region $\Omega^{*}$ of the $w$-plane which is bounded by vertical lines $\Gamma_{1}^{* *}$ corresponding to $\Gamma_{1}$ and the $x$-axis and by horizontal lines $\Gamma_{2}^{*}$ corresponding to $\Gamma_{2}$. The conformal transformation (3.34) leaves the conditions (3.29), (3.30) and (3.31) invariant, but it brings (3.32) into the simpler form

$$
\frac{\partial H}{\partial v}+H=0, \quad w=u+i v,
$$

without a variable coefficient. Hence the harmonic function

$$
H^{*}=\frac{\partial H}{\partial v}+H
$$

solves a Dirichlet problem in $\Omega^{*}$ with the single boundary condition $H^{*}=0$, since the condition (3.31) appears only on the vertical lines $\Gamma_{1}^{*}$ and can therefore be differentiated with respect to $v$. Thus we can find $H^{*}$ in closed form with the help of the Schwarz-Christoffel transformation, and it is then an elementary problem to integrate the ordinary differential equation (3.36) determining the desired Green's function $H$.

We carry out the details of the above procedure for calculating $H$ in the case where the logarithmic hodograph region $\Omega^{*}$ is the semiinfinite strip

$$
0<u<\frac{\pi}{2}, \quad v>0
$$

This hodograph will occur when we study the vena contracta and the infinite cavity. In the strip (3.37), we denote by $G$ the Green's function which satisfies everywhere the usual boundary condition $G=0$, and we introduce analytic functions $P$ and $Q$ of the complex variable $w$ and the parameter $t$ such that

$$
G(w, t)=\mathscr{R}\{P(w, t)\}, \quad H(w, t)=\mathscr{R}\{Q(w, t)\} .
$$

In terms of these functions, (3.36) takes the explicit form

$$
\frac{d Q}{d w}-i Q=i \frac{\partial P}{\partial \tau}-i P
$$

where $\tau$ is the imaginary part of $t$. We can integrate (3.39) to obtain the representation 


$$
Q(w, t)=i e^{i w} \int e^{-i w}\left(\frac{\partial P(w, t)}{\partial \tau}-P(w, t)\right) d w
$$

for $Q$ in terms of $P$. One verifies easily that

$$
P(w, t)=\log \frac{\cos 2 w-\cos 2 \bar{t}}{\cos 2 w-\cos 2 t},
$$

and thus (3.38) and (3.40) yield the explicit formula

$$
\begin{aligned}
H(w, t)=\mathscr{R}\left\{\log \frac{\cos 2 w-\cos 2 \bar{t}}{\cos 2 w-\cos 2 t}\right. & +2 e^{i(w+t)} \log \operatorname{th} \frac{w+t}{2 i} \\
& \left.-2 e^{i(w-\bar{t})} \log \operatorname{th} \frac{w-\bar{t}}{2 i}\right\}
\end{aligned}
$$

for the Green's function $H$ of the strip (3.37), where th $z$ stands for the hyperbolic tangent of $z$.

In applying the perturbation series (3.11) to study specific models of free surface flow, we must impose suitable normalizations on $U$, either at infinity or at the point of separation, in order to obtain a well-defined answer. Thus we need to investigate the variation of the separation point under changes in the value of $\varepsilon$. According to (3.2), the first order normal displacement on of the free boundary $\Gamma_{2}$ which results from infinitesimal deviations in $\varepsilon$ from the value $\varepsilon=0$ is given by the expression

$$
\delta n=-\varepsilon U_{1}=-\varepsilon \psi_{1} .
$$

The corresponding vertical shift $\delta y$ in $\Gamma_{2}$ is therefore

$$
\delta y=\frac{\delta n}{\dot{x}}=-\frac{\varepsilon \psi_{1}}{\dot{x}} .
$$

We denote by $Y$ the ordinate at the point of separation and we assume that the tangent to $\Gamma$ there is vertical. For this case we derive from (3.44) the relation

$$
\frac{\partial Y}{\partial \varepsilon}=-\lim _{s \rightarrow 0} \frac{\psi_{1}}{\dot{x}}
$$

at $\varepsilon=0$, since $s$ vanishes, by definition, at the point of separation. The conformal transformation (3.34) allows us to bring (3.45) into the form

$$
\frac{\partial Y}{\partial \varepsilon}=-\frac{\partial \psi_{1}^{\prime}}{\partial u},
$$

since by hypothesis $\dot{x}=\sin u \rightarrow 0$ as $s \rightarrow 0$. In practice (3.46) is more 
useful than (3.45) because, while the functions governing the flow have regular series expansions in the $w$-plane at the point of separation, the corresponding developments proceed in powers of the square root of the argument in the physical plane. We notice that the formula (3.46) yields the first order tangential shift of the point of separation in the case of a general slope for $\Gamma$. We do not derive the analogous expressions for higher order shifts because we shall not have occasion to use them.

We close this section with a few remarks about the scope of the perturbation method we have just described. It can be seen that the technique is not restricted to the case of polygonal fixed boundaries $\Gamma_{1}$, since the shape of $\Gamma_{1}$ can be made to depend on the parameter $\varepsilon$ in such a way that, while $\Gamma_{1}$ is polygonal at the initial stage $\varepsilon=0$, it represents an arbitrary curved body when $\varepsilon=1$. They only effect of this generalization on the perturbation scheme (3.13), (3.14), (3.28) is to make the boundary condition (3.14) along $\Gamma_{1}$ inhomogeneous. In this generalized form, we can even modify the method to make it suitable for calculating plane free streamline flows past curved obstacles. The principal restriction on the method arises from the difficulty in evaluating terms of high order in $\varepsilon$, but we shall indicate later how to obtain any desired degree of accuracy in connection with our discussion of the Riabouchinsky finite cavity model.

4. Formulation in terms of an analytic function. In this section we discuss, for arbitrary values of $\varepsilon$, a representation of the stream function $\psi$ in terms of an analytic function $g(z)$ which serves to simplify many of the calculations arising in the application of our perturbation method. We shall not attempt to motivate the basic representation for $\psi$, since adequate derivations have already been presented in earlier papers $[4,5]$ for the typical case $\varepsilon=1$. We simply verify here by direct differentiation that the expression in terms of $g(z)$ which appears has all the characteristic properties defining $\psi$.

The equation of the free boundary curve $\Gamma_{2}$ can be written in the form

$$
\bar{z}=g(z)=g(z ; \varepsilon)
$$

for any value of the dimension parameter $\varepsilon$, where $g$ is an analytic function of the complex variable $z$ in the neighborhood of $\Gamma_{2}$. This form of the equation for $\Gamma_{2}$ arises when we substitute into the more usual equation for $\Gamma_{2}$ in terms of $x$ and $y$ the values $x=(z+\bar{z}) / 2$ and $y=(z-\bar{z}) / 2 i$ and solve for $\bar{z}$. We shall establish that the stream function $\psi$ for the axially symmetric free surface flow past $\Gamma_{2}$ in space of $\varepsilon+2$ dimensions is given by the fundamental formula 


$$
\begin{aligned}
& \psi(x, y ; \varepsilon)= \\
& =\mathscr{S}\left\{\begin{array} { l } 
{ 1 } \\
{ 2 ^ { \mathrm { \varepsilon } } }
\end{array} \int _ { z _ { 0 } } ^ { z } [ \overline { z } - t ] ^ { \varepsilon / 2 } [ z - g ( t ; \varepsilon ) ] ^ { \varepsilon / 2 } F \left[-\frac{\varepsilon}{2},-\frac{\varepsilon}{2} ; 1 ;\right.\right. \\
& \left.\left.\frac{(z-t)(\bar{z}-g(t ; \varepsilon))}{(\bar{z}-t)(z-g(t ; \varepsilon))}\right] g^{\prime}(t ; \varepsilon)^{1 / 2} d t\right\}
\end{aligned}
$$

in terms of the analytic function $g(z ; \varepsilon)$ and the hypergeometric series

$$
F\left[-\frac{\varepsilon}{2},-\frac{\varepsilon}{2} ; 1 ; W\right]=\frac{1}{\Gamma(-\varepsilon / 2)^{2}} \sum_{j=0}^{\infty} \frac{\Gamma(j-\varepsilon / 2)^{2}}{\Gamma(j+1)^{2}} W^{j},
$$

where $z_{0}$ is any fixed point on the free boundary $\Gamma_{2}$.

In order to prove (4.2), we use the notations (3.16) to bring the partial differential equation (3.3) for $\psi$ into the complex form

$$
L\{\psi\}=\frac{\partial^{2} \psi}{\partial z \partial \bar{z}}+\frac{\varepsilon}{2(z-\bar{z})} \partial \psi \partial-\frac{\varepsilon}{2(z-\bar{z})} \frac{\partial \psi}{\partial \bar{z}}=0
$$

If we treat $z$ as the independent variable and $t$ as a fixed parameter, we find easily that the integrand in (4.2) satisfies

$$
L\left\{[\bar{z}-t]^{\varepsilon / 2}[z-g(t ; \varepsilon)]^{\varepsilon / 2} F\left[-\frac{\varepsilon}{2},-\frac{\varepsilon}{2} ; 1 ; \frac{(z-t)(\bar{z}-g(t ; \varepsilon))}{(z-t)(z-g(t ; \varepsilon))}\right]\right\}=0,
$$

since it is essentially the Riemann function [4] associated with equation (4.4). Thus to verify that the right-hand side of (4.2) is a solution of (4.4), it will suffice to consider only differentiations involving the variation of the upper limit of integration. In applying the operator $L$, the latter differentiations yield the terms

$$
\begin{aligned}
\mathscr{S}\left\{\frac{1}{2^{\varepsilon}} \frac{\partial}{\partial \bar{z}}[\bar{z}-z]^{\varepsilon / 2}[z-g(z ; \varepsilon)]^{\varepsilon / 2} g^{\prime}(z ; \varepsilon)^{1 / 2}+\right. \\
\left.+\frac{\varepsilon}{2^{\varepsilon+1}(z-\bar{z})}[\bar{z}-z]^{\varepsilon / 2}[z-g(z ; \varepsilon)]^{\varepsilon / 2} g^{\prime}(z ; \varepsilon)^{1 / 2}\right\}=0,
\end{aligned}
$$

and therefore the function $\psi$ defined by (4.2) does satisfy the required partial differential equation (4.4). This function also vanishes on $\Gamma_{2}$, since for $z$ on $\Gamma_{2}$ we can take the path of integration to coincide with $\Gamma_{2}$, and thus the integrand will be real by (4.1). To calculate $\partial \psi / \partial n$ from (4.2) and verify the constant pressure condition (3.2), we express differentiation in the direction of the inner normal to $\Gamma_{2}$ in the form

$$
\frac{\partial}{\partial n}=i \dot{z} \frac{\partial}{\partial z}-i \overline{\dot{z}} \frac{\partial}{\partial \bar{z}} .
$$

Applying this to (4.2), we find along $\Gamma_{2}$ 


$$
\begin{aligned}
\frac{\partial \psi}{\partial n}= & \mathscr{J}\left\{\frac{i \dot{z}}{2^{\varepsilon}}[\bar{z}-z]^{\varepsilon / 2}[z-\bar{z}]^{\varepsilon / 2} g^{\prime}(z ; \varepsilon)^{1 / 2}\right. \\
& \left.+\frac{1}{2^{\varepsilon}} \int_{z_{0}}^{z} \frac{\partial}{\partial n}|\bar{z}-t|^{\varepsilon} F\left[-\frac{\varepsilon}{2},-\frac{\varepsilon}{2} ; 1 ; \frac{|z-t|^{2}}{|\bar{z}-t|^{2}}\right]|d t|\right\} \\
= & \frac{|\bar{z}-z|^{\varepsilon}}{2^{\varepsilon}}=y^{\varepsilon},
\end{aligned}
$$

since $g^{\prime}(z ; \varepsilon)=\overline{\dot{z}}^{2}$ by (4.1). This establishes that formula (4.2) respresents a solution of the Cauchy problem, with data given along $\Gamma_{2}$, which characterizes the stream function $\psi$ for the axially symmetric flow that has $\Gamma_{2}$ as its free boundary. The Cauchy-Kowalewski theorem shows the uniqueness of this determination of $\psi$, and thus the representation (4.2) is proved to be correct.

We introduce next the analytic function

$$
\zeta(z ; \varepsilon)=\Phi+i \Psi=\int_{z_{0}}^{z} g^{\prime}(t ; \varepsilon)^{1 / 2} d t,
$$

which reduces to the arc length $s$ when $z$ lies on $\Gamma_{2}$. It is clear that $\zeta$ represents the complex potential of a plane flow which has for its free boundary the meridian cross section $\Gamma_{2}$ of the free surface of our generalized axially symmetric flow. We restrict ourselves in this section to flow models for which the rigid boundary $\Gamma_{1}$ consists only of vertical lines, and we show in such cases how the free boundary problem for (4.4) can be formulated conveniently in terms of the analytic function $\zeta$ alone [4].

We choose for $z_{\jmath}$ the point of separation of $\Gamma_{2}$ from $\Gamma_{1}$, and we notice that because $\Gamma_{1}$ is a vertical line segment the boundary conditions $\psi=0$ and $\Psi=0$ along $\Gamma_{1}$ are equivalent to each other, according to the two representations (4.2) and (4.9) for $\psi$ and $\Psi$ in terms of the analytic function $g$. To verify this equivalence, we remark that the condition $\Psi=0$ on $\Gamma_{1}$ implies that $g^{\prime}(z ; \varepsilon)=\zeta^{\prime}(z ; \varepsilon)^{2}$ is real and negative there and hence that

$$
\mathscr{R}\left\{g(z ; \varepsilon)-z_{0}\right\}=\mathscr{R}\left\{z-z_{\jmath}\right\}=0
$$

for $z$ on $\Gamma_{1}$. Thus the factor multiplying $g^{\prime}(t ; \varepsilon)^{1 / 2}$ in the integrand of (4.2) is real for $z$ and $t$ on $\Gamma_{1}$, which leads in turn to the relation $\psi=0$ there.

The situation on the axis of symmetry is somewhat more involved. For $z=x$ real, we find that

$$
F\left[-\frac{\varepsilon}{2},-\frac{\varepsilon}{2} ; 1 ; \frac{(x-t)(x-g(t ; \varepsilon))}{(x-t)(x-g(t ; \varepsilon))}\right]
$$




$$
=F\left[-\frac{\varepsilon}{2},-\frac{\varepsilon}{2} ; 1 ; 1\right]=\frac{\Gamma(1+\varepsilon)}{\Gamma(1+\varepsilon / 2)^{2}},
$$

and therefore

$$
\psi(x, 0 ; \varepsilon)=\mathscr{S}\left\{\frac{\Gamma(1+\varepsilon)}{2^{\varepsilon} \Gamma(1+\varepsilon / 2)^{2}} \int_{z_{0}}^{x}[x-t]^{\varepsilon / 2}[x-g(t ; \varepsilon)]^{\varepsilon / 2 \zeta^{\prime \prime}}(t ; \varepsilon) d t\right\} .
$$

Thus the requirement that the $x$-axis appear as a streamline takes the form

$$
\mathscr{F}\left\{\int_{z_{0}}^{x}[x-t]^{\varepsilon / 2}[x-g(t ; \varepsilon)]^{\varepsilon / 2} \zeta^{\prime}(t ; \varepsilon) d t\right\}=\text { const. }
$$

This condition on the $x$-axis, together with the relations

$$
\Psi=0 \quad \text { on } \Gamma_{1}, \quad \Phi+i \Psi=s \quad \text { on } \Gamma_{22},
$$

and the differential equation

$$
\Delta \Psi=0 \quad \text { in } \Omega,
$$

comprise our formulation of the free boundary problem for (4.4) in terms of the analytic function $\zeta$.

We can apply the perturbation method described in the previous section to the boundary value problem (4.13), (4.14), (4.15) by expanding $\Psi$ in an infinite series

$$
\Psi(x, y ; \varepsilon)=\Psi_{0}(x, y)+\varepsilon \Psi_{1}(x, y)+\varepsilon^{2} \Psi_{2}(x, y)+\cdots
$$

and determining the coefficients $\Psi_{j}$ in closed form as the solutions of successive linear boundary value problems in the hodograph plane. The conditions characterizing the $\Psi_{j}$ are somewhat simpler than the conditions for the analogous coefficients $\psi_{j}$ in the expansion (3.7). Expressions for the $\psi_{j}$ in terms of the $\Psi_{j}$ can easily be obtained from (4.2). Thus evidently $\psi_{0}=\Psi_{0}$, and

$$
\psi_{1}=\Psi_{1}+\frac{1}{2} \mathscr{\Psi} \int_{z_{0}}^{z}\left[\log \frac{(\bar{z}-t)(z-g(t ; 0))}{4}\right] \zeta^{\prime}(t ; 0) d t,
$$

since $\partial F / \partial \varepsilon=0$ at $\varepsilon=0$, according to (4.3). One can take the point of view that (4.17) is merely a substitution which simplifies the boundary value problem for $\psi_{1}$. The conditions which determine $\Psi_{1}$ in the plane flow region $\Omega$ corresponding to the value $\varepsilon=0$ are

$$
\Psi_{1}=0 \quad \text { on } \Gamma_{1}, \quad \frac{\partial \Psi_{1}}{\partial n}+\kappa \Psi_{1}=0 \quad \text { on } \Gamma_{2},
$$

$$
\Psi_{1}=-\frac{1}{2} \mathscr{\int} \int_{z_{0}}^{x}[\log (x-t)(x-g(t ; 0))] \zeta^{\prime}(t ; 0) d t+\text { const. }
$$


on the $x$-axis, and

$$
\Delta \Psi_{1}=0 \quad \text { in } \Omega .
$$

These conditions can either be derived from (4.17), or they can be derived directly, by the methods of the previous section, from the basic relations (4.13), (4.14) and (4.15). They have the advantage that among them (4.19) is the only one which is inhomogeneous; in particular, the differential equation (4.20) is homogeneous, in contrast with the corresponding equation for $\psi_{1}$. Thus $\Psi_{1}$ has an especially simple representation in terms of the Green's function $H$.

Some theoretical conclusions can be drawn from our discussion of the perturbation series (4.16). To be specific, let us consider the Riabouchinsky model, for which $\Gamma_{1}$ consists of two parallel segments of equal length rising vertically from the $x$-axis. We introduce the conformal transformations

$$
\xi=z+g(z), \quad \eta=z-g(z) .
$$

For $\varepsilon=0$, the function $\xi$ maps $\Omega$ onto a half-plane overlapping the upper half-plane $\operatorname{Im} \xi>0$, with a rectangle deleted. For $\varepsilon=0$, the function $\eta$ maps $\Omega$ onto a half-plane lying a positive distance above the real axis and slit along a segment of the imaginary axis. Thus $z-g(z)$ is bounded away from zero in $\Omega$ when $\varepsilon=0$. Now if we attempt to invert the functional equation (4.2) to solve for $\Psi$ in terms of $\psi$, the only points off the $x$-axis at which the inversion could break down are the roots of the equation $z-g(z)=0$. Since $z-g(z)$ is bounded away from zero when $\varepsilon=0$, and since the boundary value problems defining the coefficients $\Psi_{j}$ of the series development (4.16) are properly set, it is reasonable to assume that $z-g(z)$ will not vanish in $\Omega$ for small values of $\varepsilon$. Hence $\zeta(z ; \varepsilon)$ will be a regular function of $z$ without singularities in the flow region $\Omega$ for small $\varepsilon$, and, indeed, this should be true as long as $\varepsilon$ remains in the half-plane (3.4) where we expect convergence of the series (4.16), rearranged in increasing powers of the more suitable parameter $\delta$ given by (3.9). Our conclusion is that the boundary value problem (4.13), (4.14), (4.15) for the analytic function $\zeta$ is actually equivalent to the free boundary problem for the equation (4.4) of generalized axially symmetric flow, without any introduction of singularities for $\zeta$ in the flow region $\Omega$. The reasons justifying this conclusion for the Riabouchinsky model are equally applicable in the cases of the infinite cavity and the vena contracta.

It seems quite remarkable that the simplest free boundary problems for axially symmetric flow can thus be reformulated in terms of an analytic function $\zeta$ alone, with all the difficulties concentrated in the non-linear boundary condition $(4,13)$ along the axis of symmetry. While 
our main interest focuses on the physically significant case $\varepsilon=1$, the analysis of the last two sections indicates the usefulness of investigating the solution $\zeta$ for any other special values of $\varepsilon$, such as $\varepsilon=0$, from which relevent information can be obtained. This remark suggests studying, in particular, the cases where $\varepsilon$ is an even integer, for example, the case $\varepsilon=2$. Here the hypergeometric series (4.3) reduces to a polynomial and our reformulation of the free boundary problem can be carried out by far more elementary substitutions. Although the simpler problems thus obtained apparently still defy explicit solution, they do serve as an additional justification for our reduction of the study of the stream function $\psi$ to a study of the analytic function $\zeta$.

We close this section by pointing out a few significant properties of axially symmetric cavities and jets which follow from the representation (4.2). First, inversion of (4.2) can be used to establish that the free boundary curve $I_{2}{ }_{2}$ is analytic [5]. Also, the hodograph method shows that $\zeta$ has an expansion about the point of separation $z_{0}$ as a power series in $\left(z-z_{0}\right)^{1 / 2}$. Hence by (4.2) the actual stream function $\psi$ has a regular development in terms of the variable $\left(z-z_{0}\right)^{1 / 2}$. Knowledge of the nature of this development will be crucial for our later numerical work when we wish to establish accurate interpolation formulas for $\psi$. Finally, a generalized difference-differential equation governing the asymptotic behavior of the free surface at infinity can be set up on the basis of the boundary conditions on $\zeta$, but the conclusions thus far obtained in this direction do not justify going into details.

5. Monotonic dependence of the free boundary. In order to interpret our numerical data and to deduce from it information about the physical problem, it will be useful to have in our possession qualitative results concerning the behavior of the flow in dependence on the parameters which appear. With this in mind, we discuss in the next two sections a theorem about monotonic expansion of the flow region $\Omega$ as the dimension parameter $\varepsilon$ increases and some asymptotic formulas expressing gross physical characteristics of a steady state cavity in terms of the cavitation parameter $\sigma$.

For the sake of simplicity, we confine ourselves again to the Riabouchinsky model, with the rigid boundary $\Gamma_{1}$ composed of two equal vertical segments rising from the $x$-axis in symmetric positions with respect to the $y$-axis. For our analysis, we shall require that the distance $2 h$ between the symmetric components of $\Gamma_{1}$ remain fixed and we shall also fix the point of separation $z_{0}$ of $\Gamma_{2}$ from $\Gamma_{1}$ and the symmetrically located point $z_{0}^{*}$ where $\Gamma_{2}$ reattaches itself to $\Gamma_{1}$. We shall attempt to show that with these normalizations the free boundary curve $\Gamma_{2}$ corresponding to a positive value of $\varepsilon$ lies below the free boundary 
curve $\Gamma_{2}$ for the case $\varepsilon=0$ of the plane flow. We shall be led to conclude that the curve $\Gamma_{2}$ shrinks monotonically downwards as $\varepsilon$ increases. In particular, for a prescribed nose diameter and cavity length, the threedimensional axially symmetric cavity must have a meridian cross section contained within the corresponding plane cavity.

On the other hand, it is easily established that for $\varepsilon \geq 0$ the free boundary $\Gamma_{2}$ is convex and hence lies above the horizontal line segment joining the point of separation $z_{0}$ to the point of reattachment $z_{0}^{*}$. The convexity of $\Gamma_{2}$ is based on the fact that for $\varepsilon \geqq 0$ the velocity $q^{2}=(\nabla \varphi)^{2}$ is subharmonic in the sense that $\Delta q^{2}+\varepsilon \partial q^{2} / y \partial y \geqq 0$. Thus $q^{2}$ attains its maximum value on $\Gamma_{2}$, because it cannot have a maximum either in the flow region $\Omega$ or on the fixed boundary $\Gamma_{1}$, since $\varphi$ can be continued across $\Gamma_{1}$ by the Schwarz reflection principle to be even in its dependence on the distance from $\Gamma_{1}$. But the condition for irrotationality of the flow can be expressed along the free streamline $\Gamma_{2}$ in the form

$$
\frac{\partial q}{\partial n}+\kappa q=0
$$

Since $q$ is identically equal to its maximum value 1 along $\Gamma_{2}$ by (3.2), we see that $\partial q / \partial n<0$ on $\Gamma_{2}$, and hence the curvature $\kappa$ is positive there by (5.1). This proves the required convexity and establishes a crude estimate on the shape $\Gamma_{2}$ may take.

In order to analyze the monotonic dependence of $\Gamma_{2}$ on $\varepsilon$, as described above, we study the sign of the infinitesimal normal displacement $\delta n$ of $\Gamma_{2}$ given for small values of $\varepsilon$ by formula (3.43). According to (4.17) we can write $\delta n=-\varepsilon \Psi_{1}$, and thus we must determine the sign of $\Psi_{1}$. We prove that $\Psi_{1}>0$ along $\Gamma_{2}$ and thus show that for small $\varepsilon$ the free boundary is lowered when $\varepsilon$ is increased.

The maximum principle is not in itself sufficient to settle the question of the sign of $\Psi_{1}$, since the boundary value problem (4.18), (4.19), (4.20) has positive eigenvalues $\lambda$ if we replace (4.18) along $\Gamma_{2}$ by the more general condition

$$
\frac{\partial \Psi_{1}}{\partial n}+\lambda \kappa \Psi_{1}=0
$$

and alter the other conditions to make them all homogeneous. However, the value of the lowest eigenvalue $\lambda$ decreases if we fix $z_{0}$ and diminish $h$ steadily, since the image of the flow in the hodograph plane is a domain which expands as $h$ diminishes and since $\lambda$ has a classical characterization through a minimum problem for the Dirichlet integral in the hodograph plane whose solution decreases under expansion of the domain. Explicit calculation shows that the lowest eigenvalue approaches 1 as 
$h \rightarrow 0$, and hence all the eigenvalues $\lambda$ exceed 1 for the actual Riabouchinsky model. Therefore we conclude that the mixed Green's function $H$ for the hodograph of the Riabouchinsky flow is positive.

The requirements that the points $z_{0}$ and $z_{0}^{*}$ of separation and reattachment remain fixed give at both these points the condition

$$
\frac{\partial \Psi_{1}}{\partial u}=0,
$$

according to the formula (3.46) for the tangential shift of the point of separation. The presence of these two critical points of $\Psi_{1}$ is compensated for by the fact that $\Psi_{1}$ grows like a factor times $y$ at infinity. Thus there are two level curves $\Psi_{1}=0$ in $\Omega$ which emanate from $z_{0}$ and $z_{0}^{*}$ and proceed either to the point at infinity or to the $x$-axis. For if this were not the case, there would exist a subregion of $\Omega$, bounded by a level curve $\Psi_{1}=0$ and an arc of $\Gamma_{2}$, in which $\Psi_{1}$ would be of one sign, and an application of Green's formula

$$
\Psi_{1}=\frac{1}{2 \pi} \int\left[\Psi_{1} \frac{\partial H}{\partial n}-H \frac{\partial \Psi_{1}}{\partial n}\right] d s
$$

to such a subregion leads to a contradiction of the inequality $H>0$. We shall establish that $\Psi_{1}<0$ on the $x$-axis, whence the above level curves terminate at infinity and $\Psi_{1}>0$ on $\Gamma_{2}$.

To show that $\Psi_{1}<0$ on the $x$-axis, it suffices according to (4.19) to show that

$$
\mathscr{I} \int_{z_{0}}^{x}[\log (x-t)(x-g(t ; 0))] \zeta^{\prime}(t ; 0) d t>0 .
$$

Because of the symmetry of the flow in the $y$-axis, it is enough to prove (5.5) when $x>0$. We can integrate along $\Gamma$ and the $x$-axis, where $\zeta^{\prime}(t ; 0) d t=d \Phi>0$, and thus we have only to verify that

$$
\arg (x-t)+\arg (x-g(t ; 0)) \geqq 0
$$

there. By (4.9) we have $g^{\prime}(t ; 0)=\zeta^{\prime}(t ; 0)^{2}$ and hence $g^{\prime}(t ; 0)$ lies between -1 and +1 on the path of integration. Therefore

$$
-\mathscr{J}\{g(t ; 0)\} \geqq \mathscr{J}\{t\} \geqq 0
$$

on this path, from which (5.6) follows. This completes the proof of the monotonic dependence of $\Gamma_{2}$ on $\varepsilon$ for sufficiently small $\varepsilon$.

If we replace $g(t ; \varepsilon)$ in the representation $(4.2)$ by the function $g(t ; 0)$, we obtain on the left the stream function $\psi^{*}$ of a generalized axially symmetric flow which has as the meridian cross section of its free surface the plane free boundary curve $\Gamma_{2}$. The stream function 
$\psi^{*}$ is seen to satisfy all the boundary conditions that we imposed on the previous flow, $\psi$, except that along the $x$-axis the condition $\psi=0$ is replaced by

$$
\psi^{*}=\mathscr{F}\left\{\frac{\Gamma(1+\varepsilon)}{2^{\varepsilon} \Gamma(1+\varepsilon / 2)^{2}} \int_{z_{0}}^{x}[x-t]^{\varepsilon / 2}[x-g(t ; 0)]^{\varepsilon / 2} \zeta^{\prime}(t ; 0) d t\right\} .
$$

By (5.6) and the evident inequality $\arg (x-t)(x-g(t ; 0))<\pi / 2$, this implies that $\psi^{*}>0$ along the $x$-axis when $\varepsilon$ lies in the interval $0<\varepsilon<4$. We can use a comparison of the stream functions $\psi$ and $\psi^{*}$, which satisfy the same differential equation (3.3), to convince ourselves that the plane free boundary $\psi^{*}=0$ lies above the axially symmetric one $\psi=0$.

To carry through this comparison, we introduce a family of intermediate flows $\tilde{\psi}$, depending on a parameter $\tilde{\varepsilon}$ in the interval $0 \leqq \tilde{\varepsilon} \leqq 1$, which are chosen so that on the $x$-axis $\tilde{\psi}=\tilde{\varepsilon} \psi^{*}$, while elsewhere each $\tilde{\psi}$ fulfills all the conditions satisfied by $\psi^{*}$ and $\psi$, including the differential equation (3.3) and the free boundary conaition (3.2). By the technique of Section 3 , we find that the derivative $\partial \tilde{\psi} / \partial \tilde{\varepsilon}$ satisfies a linear mixed boundary value problem for the equation (3.3) with the condition

$$
\frac{\partial \partial \tilde{\psi}}{\partial n \partial \tilde{\varepsilon}}+\kappa \frac{\partial \tilde{\psi}}{\partial \tilde{\varepsilon}}=0
$$

imposed along the intermediate free boundary $\Gamma_{2}$, with $\partial \tilde{\psi} / \partial \tilde{\varepsilon}=0$ on $\Gamma_{1}$, and with $\partial \tilde{\psi} / \partial \tilde{\varepsilon}>0$ on the $x$-axis. Since we fix the points $z_{0}$ and $z_{0}^{*}$ of separation and of reattachment, $\partial \tilde{\psi} / \partial \tilde{\varepsilon}$ must have critical points there, whereas at infinity $\partial \tilde{\psi} / \partial \tilde{\varepsilon}$ can grow like a factor times $y^{1+\varepsilon}$.

Our objective is to establish that the free boundary curve $\tilde{\psi}=0$ rises as $\tilde{\varepsilon}$ increases, which can be done by showing that $\partial \tilde{\psi} / \partial \tilde{\varepsilon}<0$ on $\Gamma_{2}$. In order to prove this inequality, it would be sufficient, as in our study of the sign of $\Psi_{1}$ on $\Gamma_{2}$, to show that the homogeneous boundary value problem

$$
\Delta \tilde{\psi}_{1}-\frac{\varepsilon}{y} \frac{\partial \tilde{\psi}_{1}}{\partial y}=0 \quad \text { in } \Omega, \quad \frac{\partial \tilde{\psi}_{1}}{\partial n}+\kappa \tilde{\psi}_{1}=0 \quad \text { on } \Gamma_{2},
$$

$$
\tilde{\psi}_{1}=0 \quad \text { on } \Gamma_{1}, \quad \tilde{\psi}_{1}=0 \quad \text { on the } x \text {-axis , }
$$

has no eigenfunctions $\tilde{\psi}_{1}$ in any of the intermediate flow regions $\Omega$. We shall present here a proof due to Friedrichs [3] of this uniqueness theorem for the initial case $\tilde{\varepsilon}=0$.

For the uniqueness proof, we set 


$$
p=x \psi_{x}+y \psi_{y}-(1+\varepsilon) \psi,
$$

and we note that this function satisfies the conditions (5.10) because it actually represents the first order perturbation term corresponding to an infinitesimal magnification of the flow whose stream function is $\psi$. It is clear by the maximum principle that $p>0$ in $\Omega$, since a direct calculation establishes that $p$ is nonnegative on the boundary of $\Omega$, due to the convexity of $\Gamma_{2}$. Indeed, $p=y^{2+\varepsilon} \partial(x / y) / \partial s>0$ on $\Gamma_{2}$. Thus we can express any eigenfunction $\tilde{\psi_{1}}$ for $(5.10)$ and $(5.11)$ as a product $\tilde{\psi_{1}}=p V$, and we find

$$
\begin{aligned}
\iint_{\Omega}\left(\nabla \tilde{\psi}_{1}\right)^{2} \frac{d x d y}{y^{\varepsilon}}= & \iint_{\Omega}\left[p^{2}(\nabla V)^{2}+V^{2}(\nabla p)^{2}+2 p p_{x} V V_{x}\right. \\
& \left.+2 p p_{y} V V_{y}+V^{2} p p_{x x}+V^{2} p p_{y y}-\varepsilon V^{2} \frac{p p_{y}}{y}\right] \frac{d x d y}{y^{\varepsilon}} \\
= & \iint_{\Omega} p^{2}(\nabla V)^{2} \frac{d x d y}{y^{\varepsilon}}-\int_{\Gamma_{2}} V^{2} p \frac{\partial p}{\partial n} \frac{d s}{y^{\varepsilon}}
\end{aligned}
$$

by (5.11). Now on $\Gamma_{2}$

$$
\frac{\partial p}{\partial n}+\kappa p=0
$$

whence (5.13) yields

$$
\begin{aligned}
\iint_{\Omega} p^{2}(\nabla V)^{2} \frac{d x d y}{y^{\varepsilon}} & =\iint_{\Omega}\left(\nabla \tilde{\psi}_{1}\right)^{2} \frac{d x d y}{y^{\varepsilon}}-\int_{\Gamma_{2}} \kappa \tilde{\psi}_{1}^{2} \frac{d s}{y^{\varepsilon}} \\
& =\iint_{\Omega}\left(\nabla \tilde{\psi}_{1}\right)^{2} \frac{d x d y}{y^{\varepsilon}}+\int_{\Gamma} \tilde{\psi}_{1} \frac{\partial \tilde{\psi}_{1}}{\partial n} \frac{d s}{y^{\varepsilon}}=0 .
\end{aligned}
$$

It follows that $V \equiv 0$ and therefore that no eigenfunctions for the problem (5.10), (5.11) exist.

Our argument thus far shows that $\partial \tilde{\psi} / \partial \tilde{\varepsilon}>0$ on $\Gamma_{2}$ for $\tilde{\varepsilon}=0$. If the inequality were to be violated for larger values of $\tilde{\varepsilon}$, it would be necessary for a subregion $\Omega^{*}$ of $\Omega$ bounded by an arc of $\Gamma_{2}$ and a level curve $\partial \tilde{\psi} / \partial \tilde{\varepsilon}=0$ to appear in the finite part of the plane. In this subregion $\Omega^{*}$ the derivative $\partial \tilde{\psi} / \partial \widetilde{\varepsilon}$ would be an eigenfunction $\tilde{\psi}_{1}$ satisfying conditions of the type (5.10) and (5.11).

Such a subregion $\Omega^{*}$ might form if the region $\partial \tilde{\psi} / \partial \tilde{\varepsilon}>0$ were to expand with increasing $\tilde{\varepsilon}$ and were to penetrate to the free boundary $\Gamma_{2}$. In this event, $\partial \tilde{\psi} / \partial \tilde{\varepsilon}$ would be negative in the subregion $\Omega^{*}$, which would lie entirely in one quadrant. Therefore we could obtain 
a contradiction by applying the integral identities (5.13) and (5.15) to $\Omega^{*}$ with $p=\tilde{\psi}_{x}$, since this new choice of $p$, corresponding to infinitesimal translation of the flow $\tilde{\psi}$, vanishes only on the $y$-axis and hence would be of one sign in $\Omega^{*}$. In fact, for the physically significant case $\varepsilon=1$ it can be verified by appropriate use of the maximum principle that $\tilde{\psi}_{x}>0$ for $x>0$, because $\tilde{\psi}_{x}$ has on the positive $x$-axis in this case the boundary values

$$
\tilde{\psi_{x}}=\tilde{\varepsilon} \psi_{x}^{*}=\frac{\tilde{\varepsilon}}{\pi} \int_{z_{0}}^{x} \mathscr{I}\left\{\left(\frac{x-9(t ; 0)}{x-t}\right)^{1 / 2}+\left(\frac{x-t}{x-g(t ; 0)}\right)^{1 / 2}\right\} d \Phi,
$$

according to (5.8), and the expression on the right is positive in view of the inequalities

$$
|x-g(t ; 0)| \geqq|x-t|, \pi / 2 \geqq \arg (x-g(t ; 0)) \geqq-\arg (x-t) \geqq 0,
$$

valid along the path of integration.

The only other way that a subregion $\Omega^{*}$ of $\Omega$ possessing an eigenfunction $\tilde{\psi}_{1}$ could form as $\varepsilon$ increases would be for the subregion $\Omega^{*}$ to appear at first just in the neighborhood of $\Gamma_{2}^{\prime}$. The occurrence of such a small subregion $\Omega^{*}$ leads to a contradiction because here we can apply to $\Omega^{*}$ the integral identities (5.13) and (5.15) with $p=\tilde{\psi}_{x}$ if $\Omega^{*}$ lies in a single quadrant or with $p=x \tilde{\psi}_{x}+y \tilde{\psi}_{y}-(1+\varepsilon) \tilde{\psi}$ if $\Omega^{*}$ intersects the $y$-axis, since the latter choice for $p$ is positive near the point where $\Gamma_{2}$ crosses the $y$-axis. Thus a subregion $\Omega^{*}$ of $\Omega$ for which $\partial \tilde{\psi} / \partial \tilde{\varepsilon}$ is an eigenfunction, as described above, cannot exist.

It follows that $\partial \tilde{\psi} / \partial \tilde{\varepsilon}<0$ on $\Gamma_{2}$ for any $\varepsilon \geq 0$, which proves that the free boundary $\Gamma_{2}$ rises as $\tilde{\varepsilon}$ increases. Setting $\varepsilon=1$, we see that in particular the free boundary of the three-dimensional axially symmetric cavity lies below the corresponding free boundary for a plane flow which has the same points of separation and reattachment. Although we carried out the details of the proof only for the Riabouchinsky model, the same method applies to the infinite cavity and to the vena contracta. The general statement of the result in these cases is that for a prescribed point of separation the flow region $\Omega$ expands monotonically as $\varepsilon$ increases. Thus, for example, the three-dimensional vena contracta is uniformly thicker than the corresponding two-dimensional jet.

6. Asymptotic formulas. The remarks of this section apply exclusively to three-dimensional axially symmetric cavities. For small values of the cavitation number $\sigma$, we develop asymptotic expressions for the drag coefficient and for the cavity dimensions. Some of these formulas were known to Reichardt [14], who was able to give for them 
only an empirical derivation. We shall provide for the first time here a sound theoretical derivation, and in the process we shall succeed in extending the results considerably.

We introduce once more the Riabouchinsky cavity, with the symmetrically shaped nose and tail, which we now denote by $\Gamma_{1}$ and $\Gamma_{1}{ }^{*}$, respectively, joined by a free boundary $\Gamma_{2}$. We suppose that the nose $\Gamma_{1}$ and the point of separation $z_{0}$ are fixed throughout our discussion, whereas we allow the tail $\Gamma_{1}^{*}$ and the point of reattachment $z_{0}^{*}$ to move off towards infinity. The stream function $\psi$ will have at infinity the asymptotic expansion

$$
\psi \sim \frac{y^{2}}{2(1+\sigma)^{1 / 2}}
$$

where $\sigma$ is the cavitation parameter. This normalization, which is chosen to be consistent with the constant pressure condition (3.2), is slightly unusual because the velocity at infinity varies with $\sigma$, while the velocity on the free boundary remains unchanged.

The drag coefficient $C_{D}$ is given by the formula

$$
C_{D}(\sigma)=\frac{(1+\sigma) \int_{\Gamma_{1}}\left[y-(\nabla \psi)^{2} / y\right] d y}{\int_{\Gamma_{1}} y d y},
$$

where the integrations are carried out over the nose $\Gamma_{1}$ only. This formula is applicable to the case $\sigma=0$ of the infinite cavity as well as to the case $\sigma>0$ of a finite cavity. We shall establish the asymptotic expansion

$$
C_{D}(\sigma)=(1+\sigma) C_{D}(0)+o(\sigma)
$$

for $C_{D}$ in the limit as $\sigma \rightarrow 0$, where $o(\sigma)$ represents terms such that

$$
\lim _{\sigma \rightarrow 0} \frac{o(\sigma)}{\sigma}=0 \text {. }
$$

We should emphasize that the remainder $o(\sigma)$ might be only logarithmically smaller than $\sigma$, and that the point of separation $z_{0}$ is assumed to be fixed.

For a fixed shape of the nose $\Gamma_{1}$, we shall denote by $\psi(x, y ; \sigma)$ the stream function corresponding to the flow past $\Gamma_{1}$ with cavitation parameter $\sigma$. The choice of $\sigma$ determines the location of the tail $\Gamma_{1}{ }^{*}$. In order to prove (6.3) it suffices, according to the definition (6.2), to show that as $\sigma \rightarrow 0$

$$
\psi(x, y ; \sigma)=\psi(x, y ; 0)+o(\sigma)
$$


in the finite part of the meridian plane.

As $\sigma$ decreases, the finite cavity expands and fills out the infinite cavity, while, by the maximum principle, the solution $(1+\sigma)^{1 / 2} \psi(x, y ; \sigma)$ $-\psi(x, y ; 0)$ of $(1.1)$ decreases to zero through positive values. We discuss first the possibility that there might exist a positive function $E(\sigma)$ which approaches zero so slowly as $\sigma \rightarrow 0$ that $E(\sigma) / \sigma$ becomes infinite, and yet such that the limit

$$
\psi_{1}^{*}(x, y)=\lim _{\sigma \rightarrow 0} \frac{\psi(x, y ; \sigma)-\psi(x, y ; 0)}{E(\sigma)}
$$

is a non-trivial solution of (1.1) in the flow region $\Omega$ for the infinite cavity. The limit function $\psi_{1}^{*}$ must be positive, since

$$
\lim _{\sigma \rightarrow 0} \frac{\psi(x, y ; \sigma)-\psi(x, y ; 0)}{E(\sigma)}=\lim _{\sigma \rightarrow 0} \frac{(1+\sigma)^{1 / 2} \psi(x, y ; \sigma)-\psi(x, y ; 0)}{E(\sigma)},
$$

but on the other hand it must also satisfy the boundary conditions

$$
\psi_{1}^{*}=0 \quad \text { on } \Gamma_{1}, \quad \frac{\partial \psi_{1}^{*}}{\partial n}+\kappa \psi_{1}^{*}=0 \quad \text { on } \Gamma_{2},
$$

according to $\S 3$, since it represents a first order perturbation of the infinite cavity flow. Because the point of separation $z_{0}$ has been fixed, we conclude that $\psi_{1}^{*}$ has a critical point at $z_{0}$, as in the examples discussed in $\S 5$. Thus a level curve $\psi_{1}^{*}=1$ should emanate from $z_{0}$, but this contradicts the statement that $\psi_{1}^{*}$ is positive and excludes the existence of the supposed function $E(\sigma)$.

It follows that $\psi(x, y ; \sigma)-\psi(x, y ; 0)$ approaches zero at least as rapidly as $\sigma$. Therefore we redefine $\psi_{1}^{*}$ by (6.6) with $E(\sigma) \equiv \sigma$ and proceed to establish (6.5) by proving that the new limit function $\psi_{1}^{*}$ vanishes identically. We find immediately that

$$
\psi_{\mathrm{I}}^{*}=\lim _{\sigma \rightarrow 0} \frac{(1+\sigma)^{1 / 2} \psi(x, y ; \sigma)-\underline{\psi(x, y ; 0)}}{\sigma}-\frac{1}{2} \psi(x, y ; 0) .
$$

Since the first term on the right is nonnegative, it can grow at most like a constant factor times $y^{2}$ as $y \rightarrow \infty$, whence the same follows for $\psi_{1}^{*}$. Clearly, $\psi_{1}^{*}$ is a solution of (1.1) satisfying the boundary conditions (6.8) and vanishing on the $x$-axis, and since the point of separation $z_{0}$ remains fixed, $\psi_{1}^{*}$ must have a critical point there. Hence a level curve $\psi_{1}^{*}=0$ emanates from $z_{0}$ and divides $\Omega$ into at least two subregions. None of these subregions can be bounded exclusively by the level curve $\psi_{1}^{*}=0$ and by $\Gamma_{2}$ without extending to infinity, since otherwise $\psi_{1}^{*}$ would be an eigenfunction for a problem of the type (5.10), (5.11) in the subregion in question and since the uniqueness proof based on the 
integral identities (5.13) and (5.15) applies in the present situation and excludes the existence of such an eigenfunction. The conclusion to be drawn is that a subregion of $\Omega$ bounded by a level curve $\psi_{1}^{*}=0$, by $\Gamma_{1}$, and by the $x$-axis can be found, and since $y^{-2} \psi_{1}^{*}$ remains bounded as $y \rightarrow \infty$, this outcome contradicts the maximum principle. We deduce that $\psi_{1}^{*}$ must vanish identically, and this completes the proof of (6.5) and (6.3).

For the stagnation cup, for the circular disk, and for cones of large half-angle, the formula (6.3) stands in remarkable agreement with experimental data $[2,13,14]$. All experiments exhibit a linear plot of $C_{D}$ against $\sigma$ and, with the exception of cones of small half-angle, the slope of the graph is approximately equal to the intercept with the axis of ordinates. For cones with a half-angle as low as $15^{\circ}$, however, the experimental graph remains linear, while the slope and intercept appear to be unequal [13]. In view of our strict derivation of (6.3), this discrepancy raises a question which must probably be explained by taking into account subsidiary effects.

For our discussion of the cavity dimensions, we shall use the formula (2.18) expressing the drag in terms of the virtual mass. We note in this connection that in computing the virtual mass $M$ and the drag $D$ the flow must be renormalized so that the speed at infinity is 1 , in accordance with the definitions (2.5) and (2.15). Then (2.18) can be restated in terms of the drag coefficient $C_{D}$ in the form

$$
C_{D}=\frac{3}{2 \pi} \frac{\sigma V-M}{Y^{2} h}
$$

where $Y$ is the ordinate of the point of separation $z_{0}$. Notice that the factor $\pi Y^{2}$ represents the area of the projection of the obstacle $\Gamma_{1}$ on a plane perpendicular to the direction of the flow. We recall that (6.10) is only valid when the nose $\Gamma_{1}$ is a cone or a circular disk; however, the asymptotic expressions for the cavity dimensions which we shall deduce from it are doubtless valid for more general shapes.

By Euler's momentum theorem, which amounts to the statement that the integral

$$
\int\left[\psi \frac{\partial \psi_{x}}{\partial n}-\psi_{x} \frac{\partial \psi}{\partial n}\right] \frac{d s}{y}
$$

is independent of path, we obtain for $C_{D}$ the additional formula

$$
C_{\nu}=\sigma \frac{d^{2}}{Y^{2}}-\frac{2}{Y^{2}} \int \tilde{\Psi}_{y}^{2} \frac{d y}{y},
$$

where $d$ is the ordinate of the highest point on the free boundary $\Gamma_{2}$, 
where the integral is evaluated over a semi-infinite vertical line rising from this maximum point, and where

$$
\tilde{\Psi}=(1+\sigma)^{1 / 2} \psi-y^{2} / 2
$$

is the renormalized stream function describing motion of the obstacle through the fluid at unit velocity.

In order to use formulas (6.10) and (6.12) to estimate the cavity diameter $2 d$ and the cavity length $2 h$ in terms of the cavitation parameter $\sigma$, we fit an ellipse $\Gamma^{\prime}$ into the cavity whose foci are spaced $2 h$ units apart along the $x$-axis and whose minor axis is $2 d$ units long. A very large plane cavity is approximated quite accurately by an ellipse, because for the plane flow the two conformal mappings (4.21), whose sum is $2 z$, transform the flow region $\Omega$ onto two regions bounded almost entirely by a horizontal slit in the one case and by a vertical slit in the other, and because the sum of two such slit mappings represents a transformation onto the exterior of an ellipse. For a given nose and cavity length, we have shown in $\S 5$ that the axially symmetric cavity lies inside the corresponding plane cavity, and hence its shape should be flatter. Thus it is reasonable to assume that the ellipse $\Gamma^{\prime}$ described above lies essentially inside the axially symmetric cavity.

We can express the stream function $\psi^{\prime}$ for the axially symmetric potential flow past $\Gamma^{\prime}$ in terms of a pair of elliptic coordinates $\mu$ and $\nu$ in the form [8]

$$
\psi^{\prime}=h^{2} \frac{\left(\mu^{2}-1\right)\left(1-\nu^{2}\right)}{2}\left[\begin{array}{c}
\frac{\mu}{\mu^{2}-1}-\frac{1}{2} \log \frac{\mu+1}{\mu-1} \\
\frac{\mu_{0}}{\mu_{0}^{2}-1}-\frac{1}{2} \log \frac{\mu_{0}+1}{\mu_{0}-1}
\end{array}\right),
$$

where

$$
x=h \mu \nu+\text { const., } y=h\left(\mu^{2}-1\right)^{1 / 2}\left(1-\nu^{2}\right)^{1 / 2}, \mu_{0}^{2}=1+d^{2} / h^{2},
$$

and where the level curve $\mu=\mu_{0}$ represents the ellipse $\Gamma^{\prime}$ itself. For this flow we find by explicit calculation that as $h \rightarrow \infty$

$$
\int\left(\psi_{y}^{\prime}-y\right)^{2} \frac{d y}{y} \sim \frac{d^{\sharp}}{h^{2}} \int_{1}^{\infty}\left[\frac{1}{2} \log \frac{\mu+1}{\mu-1}-\frac{1}{\mu}\right]^{2} \mu d \mu,
$$

where the integral on the left is evaluated over the semi-infinite vertical line segment which terminates at the top of the minor axis. Since the functions $\tilde{\Psi}_{y}$ and $\psi_{y}^{\prime}-y$ should be of comparable magnitude, we deduce from (6.12) and (6.15) the asymptotic relation

$$
C_{D}=\frac{\sigma d^{2}}{Y^{2}}+O\left(\frac{d^{4}}{h^{2} Y^{2}}\right),
$$


where the term $O\left(d^{4} / h^{2} Y^{2}\right)$ denotes an expression which remains bounded, as $h \rightarrow \infty$, after division by $d^{4} / h^{2} Y^{2}$.

Because the ellipse $\Gamma^{\prime}$ is essentially contained within the cavity with dimensions $d$ and $h$, we can assume by the maximum principle that $\psi^{\prime}-(1+\sigma)^{1 / 2} \psi$ is positive in the flow region $\Omega$. Thus at the summit of the cavity, which coincides with the upper end of the minor axis of the ellipse, we find for large $h$ the estimate

$$
(1+\sigma)^{1 / 2}=\frac{1}{y} \partial n(1+\sigma)^{1 / 2} \psi \leqq \frac{1}{y} \frac{\partial \psi^{\prime}}{\partial n}=1+\frac{\log \frac{\mu_{0}+1}{\mu_{0}-1}-\frac{2}{\mu_{0}}}{\frac{2 \mu_{0}}{\mu_{0}^{2}-1}-\log \frac{\mu_{0}+1}{\mu_{0}-1}},
$$

since $\psi^{\prime}-(1+\sigma)^{1 / 2} \psi$ vanishes there. Hence for large $h$

$$
\sigma \leqq \frac{d^{2}}{h^{2}} \log \frac{h^{2}}{d^{2}}+O\left(\frac{d^{2}}{h^{2}}\right)
$$

On the other hand, the virtual mass $M$ for the cavity should exceed the virtual mass of the ellipse, whence

$$
\begin{aligned}
M & \geq \frac{4 \pi h d^{2}}{2} \frac{\left(\mu_{0}^{2}-1\right) \log \frac{\mu_{0}+1}{\mu_{0}-1}-2\left(\mu_{0}-1\right)}{2 \mu_{0}-\left(\mu_{0}^{2}-1\right) \log \frac{\mu_{0}+1}{\mu_{0}-1}} \\
& \geqq \frac{2 \pi}{3} \frac{d^{4}}{h} \log \frac{h^{2}}{d^{2}}+O\left(\frac{d^{ \pm}}{h}\right) .
\end{aligned}
$$

We can substitute $(6.19)$ into $(6.10)$ to obtain

$$
C_{D} Y^{2} h \leqq \frac{3}{2 \pi} \sigma V-\frac{d^{4}}{h} \log \frac{h^{2}}{d^{2}}+O\left(\frac{d^{ \pm}}{h}\right) \text {. }
$$

Since the free boundary $\Gamma_{2}$ is convex, we have $V \leqq 2 \pi d^{2} h$, whence

$$
\begin{aligned}
C_{D} Y^{2} h & \leqq 3 \sigma d^{\prime \prime} h-\frac{d^{4}}{h} \log \frac{h^{2}}{d^{2}}+O\left(\frac{d^{ \pm}}{h}\right) \\
& \leqq 3 C_{D} Y^{2} h-\frac{d^{4}}{h} \log \frac{h^{2}}{d^{2}}+O\left(\frac{d^{4}}{h}\right)
\end{aligned}
$$

by (6.16), or

$$
2 C_{D} Y^{2} h^{2} \geqq d^{4} \log \frac{h^{2}}{d^{2}}+O\left(d^{4}\right)
$$

Therefore as $h \rightarrow \infty$ 


$$
\frac{d^{4}}{h^{2} Y^{2}}=O\left(\frac{1}{\log h^{2} / d^{2}}\right) \rightarrow 0,
$$

and (6.16) can be refined to yield the asymptotic formula

$$
\frac{d^{2}}{Y^{2}} \sim \frac{C_{D}}{\sigma}
$$

expressing the maximum width $2 d$ of the cavity in terms of the cavitation parameter $\sigma$ for small values of $\sigma$.

From (6.22) and (6.24) we derive

$$
\frac{d^{2}}{h^{2}} \log \frac{h^{2}}{d^{2}} \leqq 2 \sigma+O\left(\frac{d^{2}}{h^{2}}\right),
$$

and by comparing this with (6.18) we deduce that there is a constant $B \geq 1$ such that

$$
\frac{d^{2}}{h^{2}} \log \frac{h^{2}}{d^{2}} \sim B \sigma
$$

Thus according to $(6.24)$

$$
\frac{h^{2}}{Y^{2}} \sim \frac{C_{D}}{B \sigma^{2}} \log \frac{1}{\sigma} .
$$

To evaluate $B$ we have to differentiate (6.10) with respect to $\sigma$. From (2.12) we find

$$
\sigma \frac{\partial V}{\partial \sigma}-\frac{\partial M}{\partial \sigma}=2 \pi Y^{2} C_{D} \frac{\partial h}{\partial \sigma},
$$

and hence multiplication of $(6.10)$ by $h Y^{2}$, followed by differentiation with respect to $\sigma$, yields

$$
Y^{2} C_{D} \frac{\partial h}{\partial \sigma}+Y^{2} h \frac{\partial C_{D}}{\partial \sigma}=\frac{3 V}{2 \pi}+3 Y^{2} C_{D} \frac{\partial h}{\partial \sigma} .
$$

If we consider only terms in (6.29) of the lowest order in $\sigma$ and if we note that according to (6.27) we have $\partial h / \partial \sigma \sim-h / \sigma$, we can derive from (6.29) and (6.24) the asymptotic identity

$$
V \sim \frac{4 \pi}{3} h d^{2}
$$

Substituting this result into (6.20), we find

$$
\begin{aligned}
C_{D} & \leqq \lim _{\sigma \rightarrow 0}\left[\frac{2 \sigma d^{2}}{Y^{2}}-\frac{d^{4}}{Y^{2} h^{2}} \log \frac{h^{2}}{d^{2}}\right] \\
& \leqq 2 C_{D}-B C_{D},
\end{aligned}
$$


by (6.26). Hence $B \leq 1$ and we must have, in fact, $B=1$. Thus we can bring (6.26) and (6.27) into the final form

$$
\frac{h^{2}}{Y^{2}} \sim \frac{C_{D}}{\sigma^{2}} \log \frac{1}{\sigma}, \frac{h^{2}}{d^{2}} \sim \frac{1}{\sigma} \log \frac{1}{\sigma} .
$$

The formulas (6.32), together with (6.24) and (6.3), serve to describe the three flow quantities $C_{D}, d / Y$ and $h / Y$ asymptotically for small values of $\sigma$ in terms of the single numerical factor $C_{D}(0)$ associated with the shape of the obstacle $\Gamma_{1}$. It is consistent with physical intuition that the ratio $h / d$ is asymptotically independent of the nose shape altogether. The fact that the numerical factor $C_{D}(0)$ which appears is to be calculated directly from the infinite cavity flow justifies our use of the special Riabouchinsky finite cavity model and shows that the introduction of the artificial tail $\Gamma_{1}^{*}$ need not disturb us. Thus nothing essentially new would ensue if we were to study instead, for example, the re-entrant jet model.

In closing this section we carry out a crude check on the asymptotic expressions (6.24) and (6.32) for the finite cavity dimensions $d$ and $h$ by comparing them with Levinson's asymptotic formula [10]

$$
y \sim 2^{1 / 2} Y^{1 / 2} C_{D}^{1 / 4} \frac{x^{1 / 2}}{(\log x)^{1 / 4}}
$$

for the shape of the free boundary $\Gamma_{2}$ of the infinite cavity. Since the finite cavity is contained within the infinite cavity for a given nose $\Gamma_{1}$, we should find when we set the abscissa $x$ in (6.33) equal to the distance $h$ from the nose to the center of the finite cavity that the resulting ordinate $y$ exceeds the maximum altitude $d$ of the finite cavity. Thus according to (6.33) we should obtain for large values of $h$ the inequality

$$
d \leqq 2^{1 / 2} Y^{1 / 2} C_{D}^{1 / 4} \frac{h^{1 / 2}}{(\log h)^{1 / 4}}
$$

Actually, (6.24) and (6.32) combine to show that

$$
d \sim Y^{1 / 2} C_{D}^{1 / 4} \frac{h^{1 / 2}}{(\log h)^{1 / 1}},
$$

which is in agreement with the estimate (6.34). It is interesting that the coordinate $y$ of the infinite cavity exceeds the altitude $d$ of the finite cavity asymptotically by the simple factor $2^{1 / 2}$

7. Calculation of the contraction coefficient. We turn in this section to the numerical calculation of the contraction coefficient $C_{c}$ for the axially symmetric vena contracta. In the meridian plane, our model 
is composed of a flow region $\Omega$ bounded by the $x$-axis, by a rigid wall $\Gamma_{1}$ consisting of the semi-infinite segment $y \geq Y, x=0$, and by a free boundary curve $\Gamma_{2}$ which separates from $\Gamma_{1}$ at the point $z_{3}=i Y$ and proceeds to the right, descending to fit at infinity the horizontal asymptote $y=X$. For three-dimensional flow, the contraction coefficient $C_{c}$ that we must compute is defined by the formula

$$
C_{c}=\frac{X^{2}}{Y^{2}}
$$

Our method of calculating $C_{c}$ is based on a consideration of the above vena contracta model for generalized axially symmetric flows $\psi$ satisfying equation (3.3), and on the introduction of the corresponding contraction coefficient

$$
C_{c}(\varepsilon)=X(\varepsilon)^{1+\varepsilon} / Y(\varepsilon)^{1+\varepsilon},
$$

which depends on the dimension parameter $\varepsilon$. The generalized stream function $\psi=\psi(x, y ; \varepsilon)$ is a bounded solution of (3.3) in $\Omega$ satisfying the boundary conditions

$$
\begin{gathered}
\psi=0 \quad \text { on } \Gamma_{1}+\Gamma_{2}, \quad \frac{1}{y^{\varepsilon}} \frac{\partial \psi}{\partial n}=1 \quad \text { on } \Gamma_{2}, \\
\psi=X^{1+\varepsilon} /(1+\varepsilon) \quad \text { for } y=0 .
\end{gathered}
$$

To supplement the perturbation scheme outlined in $\S \S 3$ and 4 , we shall determine the vena contracta explicitly in the degenerate cases $\varepsilon=-1$ and $\varepsilon=+\infty$.

For $\varepsilon \geqq 0$ it can be shown by the method of $\S 5$ that the free boundary $\Gamma_{2}$ of the vena contracta is a convex curve. The proof, which is based on (5.1), will not be repeated here. It follows that $\Gamma_{2}$ lies below that line $y=Y$, but $\Gamma_{2}$ must rise towards this line as $\varepsilon$ increases, by the monotonicity theorem of $\S 5$. To study what happens in the limit as $\varepsilon \rightarrow+\infty$, it is best to consider the velocity potential $\varphi$, which satisfies the partial differential equation

$$
\Delta \varphi+\frac{\varepsilon}{y} \varphi_{y}=0
$$

In the limiting case $\varepsilon=+\infty$, (7.5) reduces to the first order equation $\varphi_{y}=0$, which states that $\varphi$ is a function of $x$ alone. If we calculate $\varphi$ for finite values of $\varepsilon$ at points inside the jet and allow $x$ to approach $+\infty$, we find for $\varphi$ by (7.3) the behavior $\varphi \sim x$. From these remarks we conclude that

$$
\lim _{\varepsilon \rightarrow+\infty} \varphi(x, y ; \varepsilon)=x
$$


inside the jet. Thus as $\varepsilon \rightarrow+\infty$ the free boundary curve $\Gamma_{2}$ approaches the straight line $y=Y$ and $X \rightarrow Y$, whence we have

$$
\frac{X(+\infty)}{Y(+\infty)}=1
$$

However, because of the occurrence of the exponent $1+\varepsilon$ in the definition (7.2), we can deduce nothing from (7.7) about the value of $C_{c}(+\infty)$.

The situation when $\varepsilon \rightarrow-1$ is even simpler to analyze. We set $\theta=\tan ^{-1}(y / x)$ and we note that for each value of $\varepsilon$ the function

$$
\psi^{\prime}=\frac{X^{1+\varepsilon} \int_{\pi / 2}^{\theta} \sin ^{\varepsilon} \theta_{1} d \theta_{1}}{(1+\varepsilon) \int_{\pi / 2}^{\pi} \sin ^{\varepsilon} \theta_{1} d \theta_{1}}
$$

is a solution of (3.3) representing flow from a source at the origin to a sink at infinity. Since evidently

$$
\lim _{\varepsilon \rightarrow-1}(1+\varepsilon) \int_{\pi / 2}^{\pi} \sin ^{\varepsilon} \theta_{1} d \theta_{1}=\lim _{\varepsilon \rightarrow-1} \frac{\int_{\pi / 2}^{\pi} \sin ^{\varepsilon} \theta_{1} d \theta_{1}}{\int_{\pi / 2}^{\pi} \sin ^{\varepsilon} \theta_{1} \cos \theta_{1} d \theta_{1}}=1,
$$

we find

$$
\begin{aligned}
\psi^{\prime}(x, y ;-1) & =\lim _{\varepsilon \rightarrow-1} \frac{X^{\varepsilon+1} \int_{\pi / 2}^{\theta} \sin ^{\varepsilon} \theta_{1} d \theta_{1}}{(1+\varepsilon) \int_{\pi / 2}^{\pi} \sin ^{\varepsilon} \theta_{1} d \theta_{1}}=\int_{\pi / 2}^{\theta} \frac{d \theta_{1}}{\sin \theta_{1}} \\
& =\log \frac{y}{\left(x^{2}+y^{2}\right)^{1 / 2}+x} .
\end{aligned}
$$

Thus for $\varepsilon=-1$ the entire $y$-axis appears as a free boundary for the flow with stream function $\psi^{\prime}$, since

$$
y \frac{\partial \psi^{\prime}}{\partial n}=-y \psi^{\prime}=1
$$

there. Hence we must choose for $\Gamma_{2}$ the segment $0 \leqq y \leqq Y, x=0$ in this case, and the stream function $\psi=\psi^{\prime}$ will satisfy all the requirements (7.3) and (7.4) for the vena contracta flow. The conclusion to be drawn is that the jet becomes quite narrow as $\varepsilon$ decreases and degenerates altogether in the limiting case $\varepsilon=-1$, so that

$$
\frac{X(-1)}{Y(-1)}=0 \text {. }
$$


We can also calculate the limit of $C_{c}(\varepsilon)$ as $\varepsilon \rightarrow-1$. For generalized axially symmetric flows, Euler's momentum theorem remains valid because the integral

$$
\int\left[\psi^{\left.\frac{\partial \psi_{x}}{\partial n}-\psi_{x} \frac{\partial \psi}{\partial n}\right] \frac{d s}{y^{\varepsilon}}}\right.
$$

is independent of path. From the momentum theorem one deduces in a standard way the formula

$$
2 X^{1+\varepsilon}=Y^{1+\varepsilon}+(1+\varepsilon) \int_{Y}^{\infty} \psi_{x}^{2} \frac{d y}{y^{\varepsilon}},
$$

where the integral on the right is evaluated along the $y$-axis. To estimate this integral, we note that the solution $\psi-\psi^{\prime}$ of (3.3) is positive in the second quadrant, by the maximum principle. Since $\psi=\psi^{\prime}=0$ for $x=0, y \geq Y$, we conclude that

$$
-\psi_{x}=\frac{\partial \psi}{\partial n} \geqq \frac{\partial \psi^{\prime}}{\partial n}=-\psi_{x}^{\prime}
$$

there. Substituting this bound into the integral in (7.14), we find by (7.8) that

$$
2 C_{c}(\varepsilon) \geqq 1+C_{c}(\varepsilon)^{2} \frac{\left(\int_{\pi / 2}^{\pi} \sin ^{\varepsilon} \theta_{1} \cos \theta_{1} d \theta_{1}\right)^{2}}{\left(\int_{\pi / 2}^{\pi} \sin ^{\varepsilon} \theta_{1} d \theta_{1}\right)^{2}},
$$

while obviously $C_{c}(\varepsilon) \leqq 1$. Letting $\varepsilon \rightarrow-1$, we conclude from (7.16) that $2 C_{c}(-1) \geqq 1+C_{c}(-1)^{2}$, whence

$$
C_{c}(-1)=1 \text {. }
$$

At first sight, the two calculations (7.12) and (7.17) may appear to be contradictory. However, these values of $C_{c}$ and $X / Y$ are consistent with the occurrence of the exponent $1+\varepsilon$ in (7.2) and they indicate a reasonable behavior of both quantities in the neighborhood of $\varepsilon=-1$. The monotonicity theorem of $\S 5$ shows that $X / Y$ is an increasing function of $\varepsilon$. The fact that the lower bound (7.16) on $C_{c}(\varepsilon)$ decreases as $\varepsilon$ increases is an indication that $C_{c}(\varepsilon)$ is a decreasing function of $\varepsilon$, and, at any rate, $(7.17)$ proves that $C_{c}(\varepsilon)$ does not remain constant.

To complete our analysis of $C_{c}(\varepsilon)$, we must determine explicitly the plane jet corresponding to the value $\varepsilon=0$. The plane flow is discribed by the complex potential $\zeta(z)$ and the analytic function $g(z)$ defined by (4.9) and (4.1) with $\varepsilon=0$. One establishes readily by the hodograph method, or alternately by an investigation of the conformal mappings 
(4.21), that $z$ and $g$ are given in terms of $\zeta$ by the relations

$$
\begin{aligned}
& z=i-\zeta+\frac{2 i}{\pi} e^{\pi \zeta / 2}-\frac{2}{\pi}\left(1-e^{\pi \zeta}\right)^{1 / 2}+\frac{2}{\pi} \log \left[1+\left(1-e^{\pi \zeta}\right)^{1 / 2}\right], \\
& g=-i-\zeta-\frac{2 i}{\pi} e^{\pi \zeta / 2}-\frac{2}{\pi}\left(1-e^{\pi \zeta}\right)^{1 / 2}+\frac{2}{\pi} \log \left[1+\left(1-e^{\pi \zeta}\right)^{1 / 2}\right],
\end{aligned}
$$

if we normalize so that $X=1$ and if we choose the sign of $\zeta$ so that the flow moves in a reversed direction from the vena contracta back into the left half-plane. With this normalization, $\zeta$ is negative on the free boundary $\Gamma_{2}$ and represents the arc length there measured from the point of separation with the region $\Omega$ on the left.

We verify by direct inspection of (7.18) the classical result

$$
C_{c}(0)=\frac{X(0)}{Y(0)}=\frac{\pi}{\pi+2}=.61102 \text {. }
$$

Before applying the perturbation method in order to compute the derivative of $X / Y$ with respect to $\varepsilon$, we make a first estimate of $X(1) / Y(1)$ using only the more elementary data (7.7), (7.12) and (7.20). In $\S 3$ we indicated that $\psi$ is a regular function of $\varepsilon$ in the half-plane (3.4) and therefore is a regular function of the more suitable variable $\delta=\varepsilon /(\varepsilon+2)$ in the unit circle $|\delta|<1$. Thus the most accurate way to calculate $X(1) / Y(1)$ from the data (7.7), (7.12), (7.20) would seem to be interpolation based on an approximation for $X / Y$ in the form of a quadratic polynomial in $\delta$. The expression

$$
X / Y=\frac{\pi}{\pi+2}\left(1-\delta^{2}\right)+\frac{1}{2}\left(\delta+\delta^{2}\right)
$$

fits all the data (7.7), (7.12), (7.20). Since $\varepsilon=1$ corresponds to $\delta=1 / 3$, this yields for $X(1) / Y(1)$ the approximate value .765 . By definition (7.1), we can derive from this the preliminary numerical result

$$
C_{c}(1)=.586
$$

It is remarkable that an answer as accurate as (7.22) can be found for the contraction coefficient $C_{c}$ of the three-dimensional vena contracta by calculations which scarcely require pencil and paper.

We determine next the derivative of $X / Y$ with respect to $\varepsilon$ at $\varepsilon=$ 0 . For this purpose, we normalize the flow so that $X=1$ for all $\varepsilon$. Thus the problem reduces to calculating $\partial Y / \partial \varepsilon$, which can be done by an application of (3.46). By (4.17) it is permissible to replace $\psi_{1}$ in (3.46) by the more convenient perturbation term $\Psi_{1}$ and to use the revised formula 


$$
\frac{\partial Y}{\partial \varepsilon}=-\frac{\partial \Psi_{1}}{\partial u}
$$

The term $\Psi_{1}$ can be found in the logarithmic hodograph image $\Omega^{*}$ of the plane flow region $\Omega$, which is easily seen to be the semi-infinite strip (3.37) in the present example, after suitable translation. Since all the conditions (4.18), (4.19) and (4.20) determining $\Psi_{1}$ are homogeneous, with the exception of (4.19), we can express $\Psi_{1}$ in terms of the Green's function (3.42) in the form

$$
\Psi_{1}(u, v)=\frac{1}{2 \pi} \int_{0}^{\infty} \Psi_{1}(0, \tau) \frac{\partial H(u+i v, i \tau)}{\partial n} d \tau,
$$

where the normal derivative is taken with respect to the second argument of $H$. Since the point of separation corresponds in the hodograph plane to the point $w=\pi / 2$, we can substitute (7.24) into (7.23) to obtain by $(3.42)$

$$
\frac{\partial Y}{\partial \varepsilon}=\frac{1}{\pi} \int_{0}^{\infty} \Psi_{1}(0, \tau)\left[\frac{3+e^{-2 \tau}}{c h^{2} \tau}+4 e^{-\tau} \tan ^{-1} e^{-\tau}\right] d \tau
$$

It remains to calculate $\Psi_{1}(0, \tau)$ from (4.19). If we take the boundary condition (7.4) into account, we can rewrite (4.19) in the form

$$
\begin{aligned}
\Psi_{1}(0, \tau)= & \log 2-1-\frac{1}{2} \int_{0}^{1} \log |x-t||x-g(t)| d \Psi \\
& +\frac{1}{2} \int_{s^{*}}^{s} \tan ^{-1} \frac{\mathscr{F} g(t)}{x-\mathscr{R} g(t)} d \Phi,
\end{aligned}
$$

where $\zeta=\Phi+i \Psi$ is treated as the independent variable and corresponds to $z=t$, where $s+i, x$ and $i \tau$ represent corresponding points in the $\zeta-$ plane, in the $z$-plane and in the $w$-plane, respectively, and where the first integral on the right is evaluated over the level curve $\Phi=s^{*}<0$ and the last one over the level curve $\Psi=1$. Substituting the relations (7.18) and (7.19) into (7.26) and letting $s^{*} \rightarrow-\infty$, we find

$$
\begin{aligned}
\Psi_{1}(0, \tau)= & \frac{1}{2} \int_{0}^{\infty}\left\{\operatorname { c o t } ^ { - 1 } \left[\frac{\alpha}{2}+\frac{1+e^{-\pi \alpha / 2}}{\pi} e^{\pi s / 2}+\frac{\left(1+e^{\pi s}\right)^{1 / 2}-\left(1+e^{\pi s-\pi \alpha}\right)^{1 / 2}}{\pi}\right.\right. \\
& \left.\left.+\frac{1}{\pi} \log \frac{1+\left(1+e^{\pi s-\pi \alpha}\right)^{1 / 2}}{1+\left(1+e^{\pi s}\right)^{1 / 2}}\right]-\cot ^{-1} \frac{\alpha}{2}\right\} d \alpha,
\end{aligned}
$$

where $\alpha=s-\Phi$ and $e^{\pi s}=\operatorname{sh}^{2} \tau$. We introduce the new variables of integration $a=e^{-\tau}$ and $b=\alpha e^{-\tau}$ and put (7.27) into (7.25) to derive the final formula 


$$
\frac{\partial Y}{\partial \varepsilon}=-\frac{2}{\pi} \int_{0}^{1} \int_{0}^{\infty}\left[\frac{3+a^{2}}{\left(1+a^{2}\right)^{2}}+\frac{\tan ^{-1} a}{a}\right] \tan ^{-1} \frac{2 \gamma a}{4 \pi a^{2}+\pi b^{2}+\gamma b} d b d a
$$

where

$$
\begin{aligned}
\gamma=2+\left(1-a^{2}\right) e^{-\pi b / 2 a}- & {\left[4 a^{2}+\left(1-a^{2}\right)^{2} e^{-\pi b / a}\right]^{1 / 2} } \\
+ & 2 a \log \frac{2 a+\left[4 a^{2}+\left(1-a^{2}\right)^{2} e^{-\pi b / a}\right]^{1 / 2}}{(1+a)^{2}} .
\end{aligned}
$$

The integral (7.28) was evaluated by Simpson's rule on an IBM calculator. A suitable asymptotic expansion for the integrand was used for arguments $b \geqq 4$, while for the numerical integration the interval $0 \leqq b \leqq 4$ was subdivided into 40 equal parts and the interval $0 \leqq a \leqq 1$ was subdivided into 12 equal parts. The answer obtained in this manner turned out to be

$$
\frac{\partial Y}{\partial \varepsilon}=-.650544
$$

where at least the first four figures are significant.

In order to use (7.30) to improve our estimate of the contraction coefficient $C_{c}(1)$, we compute

$$
\left.\frac{\partial X}{\partial \varepsilon Y}\right|_{\varepsilon=0}=.24287
$$

With this new piece of information, we can approximate the ratio $X / Y$ by interpolating with a cubic polynomial in $\delta=\varepsilon /(\varepsilon+2)$. Indeed, using all four of the known values (7.7), (7.12), (7.20) and (7.31), we are led to the expression

$$
X / Y=.6110+.4857 \delta-.1110 \delta^{2}+.0143 \delta^{3}
$$

for $X / Y$. Since $\varepsilon=1$ corresponds to $\delta=1 / 3$, we obtain from (7.32) the result

$$
X(1) / Y(1)=.7611 \text {. }
$$

Putting this into (7.1), we obtain the final numerical value

$$
C_{c}(1)=.5793 \cong .58
$$

for the contraction coefficient $C_{c}(1)$ of the three-dimensional jet. The close agreement between the preliminary estimate (7.22) and the final answer (7.34) indicates that our procedure converges rapidly, as does also the steady decline in the magnitude of the coefficients in the expansion (7.32). The error in the first approximation (7.22) was only 1 
per cent, and the error in the final answer (7.34) can be expected to be of about the order of $1 / 10$ per cent.

Our figure .58 for the contraction coefficient $C_{c}$ is in significant disagreement with the generally accepted value .61. The theoretical justification for the value .61 stems from the early work of Trefftz [17], who advanced the conjecture that the contraction coefficients for the plane and axially symmetric cases would be identical. The result (7.17) gives evidence of the weakness of this conjecture, because it shows, together with (7.20), that $C_{c}$ must change its value as the dimension parameter $\varepsilon$ varies, whereas if Trefftz' conjecture were true one would expect $\mathrm{C}_{c}$ to remain constant. On closer examination, even Trefftz' numerical data do not necessarily indicate a value for $C_{c}$ as high as .61, and it may be that his interpretation of the data was unduly influenced by the conjecture. The work on the contraction coefficient by relaxation methods $[15,16]$ was carried through after the value .61 had been accepted as a reasonable point of departure, and it would appear that in the form in which it was applied relaxation was not sufficiently sensitive to detect the error in this value, which is only about 5 per cent.

Comparison of our answer $C_{c}=.58$ with experimental data proves to be quite interesting. Experimental values of the contraction coefficient $C_{c}$, or of the related discharge coefficient $C_{d}$, are measured for flow from a pipe of radius $Z$ through an orifice of radius $Y$, and the results are plotted against the ratio $Y \mid Z$. In the graph compiled by Lansford [9], the values of $C_{a}$ decrease steadily as $Y \mid Z$ decreases from 1 to about .24, where they reach a minimum of approximately .60 . The graph proceeds to rise again as $Y \mid Z$ descends below .24, but the experimental data terminate for $Y \mid Z$ just a little larger than .1, leaving one with the impression that in the limiting case $Y \mid Z=0$ the discharge coefficient $C_{d}$ might even exceed .61. We shall prove in a moment with complete mathematical rigor that $C_{c}$ decreases monotonically with $Y / Z$, which will establish the same result for $C_{d}$, since

$$
C_{d}=\frac{C_{c}}{\left(1-C_{c}^{2} Y^{4} / Z^{4}\right)^{1 / 2}}
$$

is a monotonic function of $\mathrm{C}_{c}$. Thus on theoretical grounds the value of $C_{d}$ could not actually rise as $Y \mid Z$ falls below .24. The disagreement here between theory and Lansford's data is undoubtedly due to the difficulties inherent in making correct experimental measurements for small values of $Y \mid Z$. In any case, if one extrapolates from the experimental graph using only values of $C_{a}$ measured for $Y \mid Z$ exceeding .24, one is led at $Y \mid Z=0$ to, for all intents and purposes, the value $C_{c}=C_{a}$ $=.58$. Our calculation (7.34) is thus in substantial agreement with this 
interpretation of the experimental data.

It remains to establish the monotonic dependence of the contraction coefficient $C_{c}=X^{2} / Y^{2}$ on the ratio $Y / Z$. For this purpose, it suffices to normalize so that $Y=1$ and then to show that $X$ decreases as $Z$ increases. Suppose, on the contrary, that there exist two pipes of radii $Z$ and $Z^{*}$, with $Z^{*}>Z$, which give rise to a pair of jets whose respective radii $X$ and $X^{*}$ at infinity stand in the relationship $X^{*} \geq X$. We denote by $\psi$ and $\psi^{*}$ the corresponding stream functions, normalized so that $\psi=\psi^{*}=1$ along the $x$-axis and so that both functions vanish on their respective free boundaries $\Gamma_{2}$ and $\Gamma_{2}^{*}$. Since $X^{*} \geqq X$, we can translate the flow region $\Omega^{*}$ in which $\psi^{*}$ is defined to the right, if necessary, until it just includes the flow region $\Omega$ in which $\psi$ is defined. In this situation we shall have $\psi^{*}>\psi$ in $\Omega$, by the maximum principle, with $\psi^{*}=\psi=0$ at the point where the free boundaries $\Gamma_{2}$ and $\Gamma_{2}^{*}$ touch. Hence at this point we find by a standard comparison argument [7]

$$
q=\frac{1}{y} \frac{\partial \psi}{\partial n}<\frac{1}{y} \frac{\partial \psi^{*}}{\partial n}=q^{*}
$$

Since $\psi=\psi^{*}=1$ on the $x$-axis, the flux through the two jets must be the same, whence

$$
q X^{2}=q^{*} X^{* 2}
$$

In view of the hypothesis $X^{*} \geqq X$, this contradicts (7.36), and we are thus led to the desired conclusion that $X^{*}<X$ when $Z^{*}>Z$.

Finally, we point out that the technique of this section can be generalized to enable us to compute the contraction coefficient for a jet issuing from a pipe of arbitrary finite radius $Z$. The results here would show that the contraction coefficients for the axially symmetric jets are slightly smaller than the contraction coefficients for the corresponding plane flows which have the same rigid boundaries in the meridian plane. By an even easier application of the method, we can calculate also the jet through a circular hole at the end of a cone, an example in which the fixed boundary $\Gamma_{1}$ appears in the meridian plane as a semi-infinite line segment with a finite slope.

8. Drag coefficient for the infinite cavity. We calculate next by the methods of $\S 7$ the drag coefficient $C_{D}$ for the infinite cavity flow past a circular disk. In the meridian plane, the fixed boundary $\Gamma_{1}$ consists of the segment $0 \leqq y \leqq Y$ of the $y$-axis, and the free boundary $\Gamma_{\text {, }}$ emanates from the point of separation $z_{0}=i Y$ and proceeds indefinitely upwards and to the right. We consider this model for all values of the dimension parameter $\varepsilon$, and thus the stream function $\psi$ will be a 
solution of (3.3) which vanishes on the entire boundary of the flow region $\Omega$, which satisfies the constant pressure condition (3.2) along $\Gamma_{2}$, and which has the asymptotic behavior

$$
\psi \sim \frac{y^{1+\varepsilon}}{1+\varepsilon}
$$

at infinity. For each value of $\varepsilon$, the drag coefficient $C_{D}=C_{D}[\varepsilon]$ is given in terms of the stream function $\psi=\psi(x, y ; \varepsilon)$ by the formula

$$
C_{D}[\varepsilon]=\frac{1+\varepsilon}{y^{1+\varepsilon}} \int_{\Gamma_{1}}\left[y^{\varepsilon}-(\nabla \psi)^{2} / y^{\varepsilon}\right] d y \text {. }
$$

In this section we consider $C_{D}$ only as a function of the parameter $\varepsilon$, and thus we use the square brackets in (8.2) to avoid confusion with the notation (6.2) for $C_{D}$ as a function of the cavitation parameter $\sigma$, which is zero in the present case. We shall estimate the physically significant value $C_{D}[1]$ of the drag coefficient for three-dimensional flow by calculating the three quantities $C_{D}[-1], C_{D}[0]$ and $C_{D}^{\prime}[0]$ and substituting them into a suitable interpolation formula for the function $C_{D}[s]$.

To evaluate $C_{D}[-1]$, it is first necessary to find a bound on $(\nabla \psi)^{2}=$ $\psi_{x}^{2}$ along the fixed boundary $\Gamma_{1}$. Since $-\psi_{x}$ is a solution of (3.3), it must assume its maximum value on the boundary of $\Omega$, and it cannot reach this maximum in the interior of $\Gamma_{1}$ because, by the Schwarz reflection principle, it can be continued across $\Gamma_{1}$ as an even function of $x$. Thus $-\psi_{x}$ attains its maximum value on $\Gamma_{2}$, and for $\varepsilon \leqq 0$ we can even state that the maximum will be achieved at the point of separation $z_{0}=i Y$, since $-\psi_{x} \leqq \partial \psi / \partial n=y^{\varepsilon}$ along $\Gamma_{2}$. Hence

$$
(\nabla \psi)^{2}=\psi_{x}^{2} \leqq Y^{\varepsilon}
$$

on $\Gamma_{1}$ for $\varepsilon \leqq 0$.

If we substitute the inequality (8.3) into (8.2), we find

$$
C_{D}[\varepsilon] \geqq 1-\frac{1+\varepsilon}{1-\varepsilon}
$$

for $\varepsilon \leqq 0$, while obviouly $C_{D}[\varepsilon] \leqq 1$ for all values of $\varepsilon$. Letting $\varepsilon \rightarrow-1$, we derive from (8.4) the final result

$$
C_{D}[-1]=1 \text {. }
$$

Unfortunately, we have not succeeded in establishing a corresponding relation for the limiting case $\varepsilon=+\infty$.

From here on we assume, without loss of generality, that $Y=1$. We must study in detail the plane flow corresponding to $\varepsilon=0$ and the 
first order perturbation terms associated with it in order to compute $C_{D}[0]$ and $C_{D}^{\prime}[0] . \quad$ As in the case of the vena contracta, the plane flow is governed by a complex potential $\zeta(z)$ and by an analytic function $g(z)$ describing the free boundary curve $\Gamma_{2}$ in the form (4.1). By the hodograph method, or from the mappings (4.21), we find that $z$ and $g$ are given in terms of $\zeta$ by the two formulas

$$
\begin{aligned}
z=\left(\zeta^{2}+\frac{2 \zeta}{4+\pi}\right)^{1 / 2} & +2 i\left(\frac{2}{4+\pi}\right)^{1 / 2}\left(\zeta+\frac{2}{4+\pi}\right)^{1 / 2} \\
& -\frac{2}{4+\pi} \log \frac{\zeta^{1 / 2}+\{\zeta+2 /(4+\pi)\}^{1 / 2}}{i\{2 /(4+\pi)\}^{1 / 2}}
\end{aligned}
$$

and

$$
\begin{aligned}
g=\left(\zeta^{2}+\frac{2 \zeta}{4+\pi}\right)^{1 / 2} & -2 i\left(\frac{2}{4+\pi}\right)^{1 / 2}\left(\zeta+\frac{2}{4+\pi}\right)^{1 / 2} \\
& -\frac{2}{4+\pi} \log \frac{\zeta^{1 / 2}+\{\zeta+2 /(4+\pi)\}^{1 / 2}}{-i\{2 /(4+\pi)\}^{1 / 2}} .
\end{aligned}
$$

We deduce immediately from (8.6) the familiar expression

$$
C_{D}[0]=\frac{2 \pi}{4+\pi}=.87980
$$

for the plane drag coefficient $C_{D}[0]$.

By differentiating (8.2) with respect to $\varepsilon$ and setting $\varepsilon=0$, we derive

$$
\begin{aligned}
C_{D}^{\prime}[0]= & \int_{0}^{1}(\log y-1) \psi_{x}^{2} d y-2 \int_{0}^{1} \psi_{x} \frac{\partial \psi_{1}}{\partial x} d y \\
= & \int_{0}^{1}(\log 4 y-1) \psi_{x}^{2} d y-2 \int_{0}^{1} \psi_{x} \frac{\partial \Psi_{1}}{\partial x} d y \\
& -\mathscr{S} \int_{0}^{1} \psi_{x} \frac{\partial}{\partial x} \int_{i}^{z}[\log (\bar{z}-t)(z-g(t))] \zeta^{\prime}(t) d t d y,
\end{aligned}
$$

where the integrals are evaluated along the $y$-axis and where $\psi_{1}=\partial \psi / \partial \varepsilon$ and $\Psi_{1}=\partial \Psi / \partial \varepsilon$ are the first order perturbation terms connected by (4.17). By Green's theorem, we have

$$
-2 \int_{0}^{1} \psi_{x} \frac{\partial \Psi_{1}}{\partial x} d y=-\pi A\left(\frac{2}{4+\pi}\right)^{1 / 2}+2 \int_{-\infty}^{0} \psi_{x y}^{\prime} \Psi_{1} d x
$$

where the integral on the right is taken over the $x$-axis and where $A$ is the coefficient defined by the asymptotic expression 


$$
\Psi_{1} \sim A\left(\frac{x+\left(x^{2}+y^{2}\right)^{1 / 2}}{2}\right)^{1 / 2}
$$

for $\Psi_{1}$ in the limit as $y \rightarrow \infty$. Using (8.10) and (4.19), we can bring (8.9) into the form

$$
\begin{gathered}
C_{D}^{\prime}[0]=-\pi A\left(\frac{2}{4+\pi}\right)^{1 / 2}-\int_{0}^{1}\left[1+\log \frac{i y-g(i y)}{2 i}\right] \psi_{x}^{2} d y \\
+\int_{0}^{1} \psi_{x} \int_{i}^{i y} \frac{i(t+g(t)) \zeta^{\prime}(t)}{(i y+t)(i y-g(t))} d t d y-\int_{-\infty}^{0} \psi_{x y} \int_{i}^{x}[\arg (x-t)(x-g(t))] \zeta^{\prime}(t) d t d x .
\end{gathered}
$$

Note that in these formulas $\psi$ represents the stream function of the plane flow.

Further reduction of (8.12) requires an expression for the coefficient $A$ in terms of the Green's function $H$ introduced in $\S 3$. In the present case, the logarithmic hodograph image $\Omega^{*}$ of the plane flow region $\Omega$ is easily seen to be the semi-infinite strip (3.37), and therefore $H$ is given again by (3.42) in terms of variables in the hodograph plane. In order to evaluate $A$, we consider the value of the partial derivative $\partial H / \partial u$ when the parameter $w=u+i v$ lies at the origin and we note that the line integral

$$
\int\left(\frac{\partial H}{\partial u} \frac{\partial \Psi_{1}}{\partial n}-\Psi_{1} \frac{\partial^{2} H}{\partial n \partial u}\right) d s
$$

taken with respect to the other argument of $H$, vanishes over any closed contour in the interior of $\Omega^{*}$. When we deform the contour of integration to bring it onto the boundary of $\Omega^{*}$, there is no contribution from the singularity of $\partial H / \partial u$ at the origin, which corresponds to the point of separation, because $\Psi_{1}$ has a critical point there, but the contribution from the singularity (8.11) of $\Psi_{1}$ amounts to a numerical factor times $A$. Thus we derive for $A$ the formula

$$
A=-\frac{1}{2 \pi}\left(\frac{2}{4+\pi}\right)^{1 / 2} \int \Psi_{1} \frac{\partial^{2} H}{\partial n \partial u} d s
$$

where the integral is evaluated over the semi-infinite vertical line segment bounding $\Omega^{*}$ on the right, which corresponds in the physical plane to the negative $x$-axis. We can substitute into (8.14) the explicit values for $H$ and for $\Psi_{1}$ given by (3.42) and by (4.19) to obtain

$$
A=\frac{1}{2 \pi}\left(\frac{2}{4+\pi}\right)^{1 / 2} \int_{0}^{\infty}\left[\frac{3+e^{-2 \tau}}{\operatorname{ch}^{2} \tau}+4 e^{-\tau} \tan ^{-1} e^{-\tau}\right] \int_{i}^{x}[\arg (x-t)(x-g(t))] \zeta^{\prime}(t) d t d \tau,
$$


where $x$ can be expressed in terms of $\tau$ by setting

$$
\zeta=-\frac{2}{4+\pi} \operatorname{coth}^{2} \tau
$$

in (8.6), according to the hodograph transformation (3.34).

The final step is to insert (8.15) into (8.12) and to replace the functions $z$ and $g$ by their explicit representations (8.6) and (8.7). In this connection, it is convenient to introduce new variables of integration. We find that

$$
C_{D}^{\prime}[0]=-I_{1}+I_{2}-I_{3}+I_{4}-I_{5}-I_{6},
$$

where

$$
I_{1}=\frac{16}{4+\pi} \int_{0}^{1}\left[1+\log \frac{2 f_{1}(b)}{4+\pi}\right] \frac{b^{2}-b^{4}}{\left(1+b^{2}\right)^{3}} d b
$$

$$
I_{2}=\frac{64}{(4+\pi)^{2}} \int_{0}^{1} \int_{0}^{1} f_{2}(a)\left[\tan ^{-1} \frac{2 f_{3}(a) f_{4}(b)}{f_{3}(a)^{2}+f_{1}(b)^{2}-f_{4}(b)^{2}}\right] \begin{gathered}
b-b^{3} \\
\left(1+b^{2}\right)^{3}
\end{gathered} d b d a
$$

$$
I_{3}=\frac{256}{4+\pi} \int_{0}^{1} \int_{a}^{1} \frac{f_{4}(b)\left\{\left(b-b^{3}\right) /\left(1+b^{2}\right)^{3}\right\}}{f_{5}(a)^{2}+f_{1}(b)^{2}-f_{4}(b)^{2}+2 f_{5}(a) f_{1}(b)},
$$

$$
I_{4}=\frac{64}{(4+\pi)^{2}} \int_{0}^{1 / 2} \int_{b}^{1} f_{2}(a) \frac{b+b^{3}}{\left(1-b^{2}\right)^{3}} \tan ^{-1} \frac{1}{f_{6}(a, b)} d a d b
$$

$$
\begin{aligned}
I_{5}=\frac{8 \pi}{(4+\pi)^{2}} \int_{1 / 2}^{1} \int_{1 / 2}^{a} f_{2}(a)\{ & f_{6}(a, b) \tan ^{-1} \frac{1}{f_{6}(a, b)} \\
& \left.+\frac{1}{2} \log \left[1+f_{6}(a, b)^{2}\right]\right\} \frac{d b d a}{b^{2}},
\end{aligned}
$$

$$
\begin{aligned}
I_{6}= & \frac{8 \pi}{(4+\pi)^{2}} \int_{1 / 2}^{1} f_{2}(a)\left\{\frac{f_{6}(a, a)}{a} \tan ^{-1} \frac{1}{f_{6}(a, a)}+\frac{1}{2 a} \log \left[1+f_{6}(a, a)^{2}\right]\right. \\
& -\log \left(1+\frac{1}{\pi^{2}}\left[\frac{4}{9}-\log 3+f_{3}(a)\right]^{2}\right) \\
& \left.-\frac{2}{\pi}\left[\frac{4}{9}-\log 3+f_{3}(a)\right] \tan ^{-1} \quad \frac{\pi}{4 / 9-\log 3+f_{3}(a)}\right\} d a
\end{aligned}
$$

with

$(8.24)$

$$
f_{1}(b)=\frac{4 b}{1+b^{2}}+\frac{\pi}{2}, \quad f_{2}(a)=\frac{4+\pi}{4}-\tan ^{-1} a-\begin{gathered}
3 a+a^{3} \\
\left(1+a^{2}\right)^{2}
\end{gathered}
$$

$$
f_{3}(a)=2 \frac{3 a-a^{3}}{\left(1-a^{2}\right)^{2}}+\log \frac{1+a}{1-a}, \quad f_{4}(b)=\tan ^{-1} \begin{gathered}
1-b^{2} \\
2 b
\end{gathered}-2 \begin{gathered}
b-b^{3} \\
\left(1+b^{2}\right)^{2}
\end{gathered},
$$




$$
\begin{gathered}
f_{.(}(a)=\tan ^{-1} \begin{array}{c}
2 a \\
1-a^{2}
\end{array}+2 \begin{array}{c}
3 a+\ell^{3} \\
\left(1+a^{2}\right)^{2}
\end{array} \\
f_{6}(a, b)=\frac{1}{\pi}\left[\begin{array}{c}
3 a-a^{3} \\
\left(1-a^{2}\right)^{2}
\end{array}+\log \frac{(1+a)(1-b)}{(1-a)(1+b)}+2 \begin{array}{c}
b-3 b^{3} \\
\left(1-b^{2}\right)^{2}
\end{array}\right] .
\end{gathered}
$$

The meaning of the variables $a$ and $b$ depends on the formulas in which they appear. We always have $a=e^{-\tau}$, where $\tau$ is the ordinate in the logarithmic hodograph plane. However, $f_{2}, f_{3}$ and $f_{6}$ occur in integrals evaluated over the negative $x$-axis and therefore when $a$ is an argument of these functions the corresponding value of $\tau$ yields through (8.16) and (8.6) an expression for $x$, whereas $f_{5}$ occurs in an integral over $I_{\text {, }}^{\prime}$ and thus when $a$ is its argument the corresponding value of $\tau$ gives by means of

$$
\zeta=-\begin{array}{r}
2 \\
4+\pi
\end{array} \operatorname{th}^{2} \tau
$$

and (8.6) an expression for the quantity iy. The variable $b$ has the same meaning that we have just ascribed to the variable $a$, except that $b$ corresponds to the point in the physical plane which we have denoted by $t$, whenever it occurs, rather than to the point $z$. The equation (8.28) must be used to find the right expression for the corresponding variable in the physical plane when $b$ is the argument of $f_{1}$ and $f_{t}$, whereas when $b$ is an argument of $f_{6}$ we must use instead the equation (8.16), because $f_{1}$ and $f_{t}$ are involved in integrals over $\Gamma_{1}$, whereas $f_{6}$ is involved in an integral over the negative real axis.

In the formula (8.17) for $C_{D}^{\prime}[0]$, the integrals $I_{1}$ and $I_{3}$ correspond to the second and third terms, respectively, on the right in (8.12), while $I_{2}, I_{4}, I_{5}$ and $I_{6}$ represent the first and last terms. In particular, integration by parts shows that

$$
I_{4}-I_{5}-I_{6}=\frac{64}{(4+\pi)^{2}} \int_{0}^{1} \int_{0}^{a} f_{2}(a) \begin{gathered}
b+b^{3} \\
\left(1-b^{2}\right)^{3}
\end{gathered} \tan ^{-1} \begin{gathered}
1 \\
f_{6}(a, b)
\end{gathered} d b d a
$$

and the only reason for breaking this integral up into three separate components is to facilitate its numerical computation.

The integrals in (8.17) were evaluated on an IBM calculator. Simpson's rule was used, with every interval subdivided into 16 equal parts. The results turned out to be

$$
\begin{array}{lll}
I_{1}=.103166, & I_{2}=.0090901, & I_{3}=.003486, \\
I_{4}=.018545, & I_{5}=.0153746, & I_{6}=-.020204 .
\end{array}
$$

Therefore the desired quantity $C_{D}^{\prime}[0]$ has the numerical value 


$$
C_{D}^{\prime}[0]=-.07419 \text {, }
$$

In order to calculate $C_{D}[1]$ from the established data (8.5), (8.8) and (8.32), it is best to interpolate by expressing $C_{D}$ as a quadratic polynomial in the variable $\delta=\varepsilon /(\varepsilon+2)$, since, as has been pointed out before, it is in terms of $\delta$ that our perturbation expansion has a maximal circle of convergence. Clearly, the formula

$$
C_{D}=.87980-.14838 \delta-.02818 \delta^{2}
$$

fits the data (8.5), (8.8) and (8.32), and, since $\delta=1 / 3$ for $\varepsilon=1$, this gives the final result

$$
C_{D}[1]=.82721 \cong .827
$$

for the drag coefficient $C_{D}[1]$ of a circular disk in the case of the infinite cavity corresponding to the value $\sigma=0$ of the cavitation parameter. We can expect the error in the answer (8.34) to be less than $1 / 2$ per cent, since this would have been the order of magnitude of the error in our earlier work on the contraction coefficient if we had used only the data (7.12), (7.20) and (7.31) and had ignored the limiting case $\varepsilon=+\infty$.

Our result $C_{D}[1]=.827$ compares quite favorably with experimental data $[2,13,14]$. The available experimental graphs of the drag coefficient $C_{D}(\sigma)$ as a function of the cavitation parameter $\sigma$ are linear, and one can extrapolate from them to determine the value of $C_{D}(0)$. One finds in this manner a spread of estimates for $C_{D}(0)$ varying between the value .79 obtained by Reichardt [14] in a free jet tunnel and the value approximately equal to .83 obtained in the high speed tunnel at the California Institute of Technology [13]. Our answer $C_{D}(0)=C_{D}[1]$ $=.827$ is thus in substantial agreement with the largest experimentally observed values of the drag coefficient. A more interesting comparison of theory with experiment ensues when we substitute the numerical result $C_{D}(0)=.827$ into the asymptotic formula (6.3) to derive for the circular disk the approximate relation

$$
C_{D}(\sigma)=.827(1+\sigma) \text {. }
$$

The line (8.35) passes directly through the midst of the experimental data, and it fits the median of the observed values in the range near $\sigma=1 / 8$, where the most experimental information is available.

It is perhaps worth commenting, in conclusion, that our method of arriving at the answer (8.34) for the drag coefficient $C_{D}$ of a circular disk extends without severe difficulty to the case of conical noses of arbitrary half-angle. It is also possible, by more elaborate modifications, to handle curved obstacles such as the sphere. 
9. Calculation of a finite cavity. The perturbation method alone does not suffice for a satisfactory analysis of finite cavities, because it gives really accurate results only for physical quantities which change relatively little in the transition from plane to axially symmetric flow, while the cavitation parameter $\sigma$ varies sharply with the dimension $\varepsilon+$ 2 for cavities of roughly the same size. Thus we shall divide our discussion of three-dimensional finite cavities into two characteristically different parts. In the present section we shall obtain by the perturbation method a first estimate of the cavity width $2 d$ for a prescribed value of the cavity length $2 h$. We shall follow this with a section devoted to a systematic iterative scheme for calculating all the desired flow quantities with successively improved accuracy.

We consider again the Riabouchinsky model, with the nose $\Gamma_{1}$ and the tail $\Gamma_{1}^{*}$ consisting of equal vertical line segments, spaced symmetrically with respect to the $y$-axis. We use the notations of $\S 6$, and we study the maximum cavity altitude $d=d(\varepsilon)$ as a function of the dimension parameter $\varepsilon$ for fixed values of the cavity length $2 h$ and of the ordinate $Y$ of the separation point. We calculate $d(+\infty), d(0)$ and $d^{\prime}(0)$, and we interpolate using these data to find $d(1)$.

The stream function $\psi=\psi(x, y ; \varepsilon)$ is a solution of (3.3) which vanishes on the $x$-axis and on $\Gamma_{1}+\Gamma_{1}^{*}+\Gamma_{2}$ and satisfies the free boundary condition (3.2) along $\Gamma_{2}$. At infinity $\psi$ has the behavior $\psi \sim y^{1+\varepsilon} /(1+\varepsilon)(1+\sigma)^{1 / 2}$. On the other hand, the velocity potential $\varphi=$ $\varphi(x, y ; \varepsilon)$ is a solution of (7.5) satisfying related boundary conditions and exhibiting the behavior

$$
\varphi \sim x /(1+\sigma)^{1 / 2}
$$

at infinity. By (7.5), the limit of $\varphi$ as $\varepsilon \rightarrow+\infty$ should be a function of $x$ alone. We conclude from (9.1) that

$$
\lim _{\varepsilon \rightarrow+\infty} \varphi(x, y ; \varepsilon)=x /(1+\sigma)^{1 / 2} .
$$

As was indicated in $\S 5$, the free boundary $\Gamma_{2}$ is a convex curve which falls steadily as $\varepsilon$ increases, and thus we deduce that $\Gamma_{2}$ collapses to the line $y=Y(+\infty)$ in the limit as $\varepsilon \rightarrow+\infty$. Therefore

$$
\frac{d(+\infty)}{Y(+\infty)}=1
$$

For $\varepsilon=0$, the Riabouchinsky flow is governed by a complex potential $\zeta(z)=\Phi+i \Psi$ and by the analytic function $g(z)$ in terms of which the free boundary $\Gamma_{2}$ has the representation (4.1). By the standard technique of conformal mapping, we find that $z$ and $g$ are given in terms of $\zeta$ by the formulas 


$$
z=\frac{1}{k} \int_{0}^{\zeta}\left(\begin{array}{c}
\zeta^{2}-k^{2} \\
\zeta^{2}-1
\end{array}\right)^{1 / 2} d \zeta+\begin{gathered}
\left(1-k^{2}\right)^{1 / 2} \\
k
\end{gathered}\left(\zeta^{2}-1\right)^{1 / 2}-\frac{1}{k} \int_{k}^{1}\left(\begin{array}{c}
\zeta^{2}-k^{2} \\
\zeta^{2}-1
\end{array}\right)^{1 / 2} d \zeta,
$$

and

$$
g=\frac{1}{k} \int_{0}^{\zeta}\left(\begin{array}{c}
\zeta^{2}-k^{2} \\
\zeta^{2}-1
\end{array}\right)^{1 / 2} d \zeta-\left(\begin{array}{c}
\left(1-k^{2}\right)^{1 / 2} \\
k
\end{array}\left(\zeta^{2}-1\right)^{1 / 2}+\frac{1}{k} \int_{k}^{1}\left(\begin{array}{c}
\zeta^{2}-k^{2} \\
\zeta^{2}-1
\end{array}\right)^{1 / 2} d \zeta\right.
$$

where $k$ is a parameter in the interval $0<k<1$ and where we have normalized so that

$$
Y=Y(0)=\frac{1-k^{2}}{k}+\frac{1}{k} \int_{k}^{1}\left(\begin{array}{c}
\zeta^{2}-k^{\prime \prime} \\
1-\zeta^{2}
\end{array}\right)^{1 / 2} d \zeta
$$

The interval $-k<\zeta<k$ corresponds to the free boundary $\Gamma_{2}$, the intervals $k<\zeta<1$ and $-1<\zeta<-k$ correspond to the nose $\Gamma_{1}$ and the tail $\Gamma_{1}^{*}$, and the remainder of the real axis in the $\zeta$-plane maps into the real axis in the physical plane.

We shall find it convenient to introduce the notations

$$
\begin{aligned}
& K=\int_{0}^{1} \frac{d \zeta}{\left(1-\zeta^{2}\right)^{1 / 2}\left(1-k^{2} \zeta^{2}\right)^{1 / 2}}, E=\int_{0}^{1}\left(\begin{array}{c}
1-k^{2} \zeta^{2} \\
1-\zeta^{2}
\end{array}\right)^{1 / 2} d \zeta, \\
& K^{*}=\int_{0}^{1} \begin{array}{c}
d \zeta \\
\left(1-\zeta^{2}\right)^{1 / 2}\left(1-k^{* 2} \zeta^{2}\right)^{1 / 2},
\end{array} \quad E^{*}=\int_{0}^{1}\left(\begin{array}{c}
1-k^{* 2} \zeta^{2} \\
1-\zeta^{2}
\end{array}\right)^{1 / 2} d \zeta, \\
& k^{*}=\left(1-k^{2}\right)^{1 / 2},
\end{aligned}
$$

for the familiar complete elliptic integrals of the first and second kinds. In terms of these quantities, we find easily from (9.4) the relations

$$
\begin{aligned}
& d(0) / Y(0)=\frac{k^{*}+E^{*}-k^{2} K^{*}}{k^{* 2}+E^{*}-k^{2} K^{*}}, \\
& h(0) / Y(0)=\frac{E-k^{* 2} K}{k^{* 2}+E^{*}-k^{2} K^{*}} .
\end{aligned}
$$

Since we fix both $h$ and $Y$ in our discussion, the cavitation parameter $\sigma=\sigma(\varepsilon)$ becomes a nontrivial function of $\varepsilon$. By (9.4)

$$
\sigma(0)=\begin{gathered}
2 k^{*} \\
1-k^{*}
\end{gathered}
$$

We can calculate the derivative $d^{\prime}(0)$ in terms of the first order perturbations $\psi_{1}=\partial \psi / \partial \varepsilon$ and $\Psi_{1}=\partial \Psi / \partial \varepsilon$ of the stream functions $\psi$ and W. By (3.44) and (4.17), we find

$$
d^{\prime}(0)=-\psi_{1}(0, d)=-\Psi_{1}(0, d),
$$


where, as indicated, the functions $\psi_{1}$ and $\Psi_{1}$ must be evaluated at the highest point on $\Gamma_{2}$. As in the earlier examples, we shall work with $\Psi_{1}$ rather than with $\psi_{1}$. We express $\Psi_{1}$ in terms of the Green's function $H$ of $\S 3$, which must be determined explicitly for the present flow.

For the Riabouchinsky model, the image $\Omega^{*}$ in the logarithmic hodograph plane of the flow region $\Omega$ is the semi-infinite strip $-\pi / 2<$ $u<\pi / 2$, slit along the semi-infinite segment $v \geqq 2^{-1} \mathrm{ch}^{-1}\left(2 k^{-2}-1\right)$ of the imaginary axis. Here we have chosen to normalize the hodograph transformation so that

$$
w=\frac{1}{i} \log \frac{d \zeta}{d z}=i \log \frac{\left(\zeta^{2}-k^{2}\right)^{1 / 2}+k^{*} \zeta}{k\left(\zeta^{2}-1\right)^{1 / 2}} .
$$

We make use of the symmetry of $\Omega$ and of $\Omega^{k}$ in the imaginary axis, and we introduce the function

$$
P^{*}(\zeta, t)=\log \frac{(\zeta-\bar{t})(\zeta+t)}{(\zeta-t)(\zeta+\bar{t})}
$$

whose real part is the symmetric sum of two classical Green's functions of $\Omega$, rather than the function $P$ of $\S 3$, whose real part is a single Green's function.

To calculate $d^{\prime}(0)$ from (9.12), it will suffice to determine the mixed Green's function $H$ for the special case where the singularity lies at the origin in the $w$-plane. However, there is still a difficulty involved in using the differential equation (3.39) to find the analytic function $Q$ whose real part is $H$, because $d Q / d w$ has a singularity at the tip of the slit along the imaginary axis bounding $\Omega^{*}$. For our application, we can overcome this difficulty by subtracting from the value of $2 Q$ with parameter point located at the origin a suitable constant factor times the sum of the derivatives of $Q$ with respect to the parameter when the latter lies at $-\pi / 2$ and at $\pi / 2$. We choose the constant factor so that the resulting symmetric expression, to be denoted by $Q_{1}$, has a finite derivative $d Q_{1} / d w$ at the tip of the slit bounding $\Omega^{*}$. For our purposes, the singularities of $Q_{1}$ at the corners $-\pi / 2$ and $\pi / 2$ of $\Omega^{*}$ will not cause trouble, since these points correspond in the physical plane to the points of separation and of reattachment, where $\Psi_{1}$ has critical points. Thus we shall be able to use $Q_{1}$, rather than $Q$, throughout what follows.

For the analytic function $Q_{1}(w)$ defined in the above manner, we obtain a differential equation

$$
\frac{d Q_{1}}{d w}-i Q_{1}=i P_{1}^{*}+(\text { const. }) P_{2}^{*}
$$


analogous to (3.39), where $P_{1}^{*}$ and $P_{2}^{*}$ are appropriate derivatives in the logarithmic hodograph plane of the function (9.14) with respect to the parameter point when the latter is located at 0 and at $\pi / 2$. The equation (9.15) can be worked out and integrated explicitly to yield

$$
Q_{1}(w)=-\frac{4 k}{k^{*}} e^{i w} \int_{\infty}^{\zeta}\left[\frac{1}{\zeta}-\frac{k^{* 2} \gamma \zeta}{\zeta^{2}-k^{2}}\right] e^{-i w} \frac{d w}{d \zeta} d \zeta,
$$

where $\zeta$ and $w$ are connected by (9.13) and where the constant $\gamma$ is determined by the condition

$$
\begin{aligned}
\int_{1}^{\infty}\left[\frac{1}{\zeta}-\frac{k^{* 2} \gamma \zeta}{\zeta^{2}-k^{2}}\right. & \left.-1+\frac{k^{* 2} \gamma}{1-k^{2}}\right] e^{-i w} \frac{d w}{d \zeta} d \zeta \\
& +i\left(1-\frac{k^{* 2} \gamma}{1-k^{2}}\right) \exp \frac{\operatorname{ch}^{-1}\left(2 k^{-2}-1\right)}{2}=0,
\end{aligned}
$$

expressing the fact that $Q_{1}(\pi / 2+i v)$ should be pure imaginary.

In order to evaluate the integral in (9.16), we introduce the Jacobi elliptic functions [11]

(9.18) $\quad \operatorname{sn} a=\mathrm{sn}(a, k), \quad \operatorname{cn} \alpha=\mathrm{cn}(a, k), \quad \operatorname{dn} a=\operatorname{dn}(a, k), \quad$ zn $a=\operatorname{zn}(a, k)$

and we make the change of variable

$$
\zeta=\frac{1}{\operatorname{sn} a}
$$

If we perform the substitution (9.19) in (9.16) and differentiate with respect to $a$, we obtain the representation

$$
\begin{aligned}
\frac{d Q_{1}}{d a} & =-\frac{4 i k \operatorname{sn} a}{\operatorname{dn} a+k^{*}}\left[k^{*} \sin ^{-1} \operatorname{sn} a-\gamma \tan ^{-1} \frac{k^{*} \operatorname{sn} a}{\operatorname{cn} a}\right. \\
& \left.+(1-2 \gamma)\left(\operatorname{zn} a+\frac{E a}{K}\right)+\gamma k^{* 2} a+2 \gamma k^{2} \frac{\operatorname{sn} a \operatorname{cn} a}{\operatorname{dn} a}+\gamma k^{2} k^{*} \frac{\operatorname{sn} a \operatorname{cn} a}{\operatorname{dn}^{2} a}\right]
\end{aligned}
$$

for the derivative $d Q_{1} / d a$, which is the actual quantity required later on. Similarly, the constant $\gamma$ can be calculated explicitly and has the value

$$
\gamma=\frac{\pi k^{*}+2 E}{\pi+4 E-2 k^{* 2} K}
$$

We set $H_{1}=\mathscr{R}\left\{Q_{1}\right\}$ and we express $\Psi_{1}(0, d)$ easily in terms of $H_{1}$ by Green's formula to derive

$$
\Psi_{1}(0, d)=\frac{1}{2 \pi} \int \Psi_{1} \frac{\partial H_{1}}{\partial n} d s,
$$


where the integral on the right is evaluated over the interval $1<\zeta<$ $\infty$ of the real axis in the $\zeta$-plane. Using (9.12) and the new variable $a$, we can bring (9.22) into the form

$$
d^{\prime}(0)=-\frac{1}{2 \pi i} \int_{0}^{K} \Psi_{1} \frac{d Q_{1}}{d a} d a .
$$

Thus it remains only to represent the boundary values of $\Psi_{1}$ along the $x$-axis in terms of the variable $a$.

We find $\Psi_{1}$ along the $x$-axis by substituting the explicit formulas (9.4) and (9.5) into the boundary condition (4.19). This yields in terms of $\zeta$ the expression

$$
\begin{array}{rl}
\Psi_{1}(\zeta)= & \frac{1}{2} \int_{k}^{1} \tan ^{-1} \frac{k^{*}\left(1-t^{2}\right)^{1 / 2}+\int_{t}^{1}\left(\begin{array}{c}
\tau^{2}-k^{2} \\
1-\tau^{2}
\end{array}\right)^{1 / 2} d \tau}{k^{*}\left(\zeta^{2}-1\right)^{1 / 2}+\int_{1}^{\zeta}\left(\begin{array}{c}
\tau^{2}-k^{2} \\
\tau^{2}-1
\end{array}\right)^{1 / 2} d \tau} d t \\
& \left.-\frac{1}{2} \int_{h}^{1} \tan ^{-1} \frac{k^{*}\left(1-t^{2}\right)^{1 / 2}+\left(\int_{k}^{1}+\int_{k}^{t}\right)\left(\frac{\tau^{2}-k^{2}}{1-\tau^{2}}\right)^{1 / 2} d \tau}{k^{*}\left(\zeta^{2}-1\right)^{1 / 2}+\int_{1}^{\zeta}\left(\tau^{2}-k^{2}\right.}\right)^{1 / 2} d \tau \\
\tau^{2}-1 & 2 \int_{k}^{1}\left(\frac{\tau^{2}-k^{2}}{1-\tau^{2}}\right)^{1 / 2} d \tau \\
& -\frac{1}{2} \int_{1}^{\zeta} \tan ^{-1} \frac{k^{*}\left(\zeta^{2}-1\right)^{1 / 2}+k^{*}\left(t^{2}-1\right)^{1 / 2}+\int_{t}^{\zeta}\left(\frac{\tau^{2}-k^{2}}{\tau^{2}-1}\right)^{1 / 2} d \tau}{d t} d t
\end{array}
$$

To reduce these integrals to a tractable form, we introduce the Jacobi elliptic functions with the complementary modulus $k^{*}$, for which we shall use the special notation

$$
\begin{cases}\mathrm{sn}^{*} b=\mathrm{sn}\left(b, k^{*}\right), & \mathrm{cn} * b=\mathrm{cn}\left(b, k^{*}\right), \\ \mathrm{dn}^{*} b=\mathrm{dn}\left(b, k^{*}\right), & \mathrm{zn}^{*} b=\mathrm{zn}\left(b, k^{*}\right) .\end{cases}
$$

In the first two integrals on the right in (9.24) we make the change of variable

$$
t=\mathrm{dn}^{*} b,
$$

while in the last integral we set

$$
t=\frac{1}{\operatorname{sn} b} .
$$

We use the relation (9.19) throughout for $\zeta$. With these substitutions completed, we can put $(9.24)$ into $(9.23)$ to obtain the final result 


$$
d^{\prime}(0)=-I_{1}-I_{3}+I_{3}-I_{4},
$$

where

$$
I_{1}=k_{4 \pi}^{* 2} \int_{0}^{K} \int_{0}^{K *} f_{1}(a) \mathrm{cn}^{*} b \mathrm{sn}^{*} b \tan ^{-1} f_{2}(a, b) d b d a,
$$

$$
I_{2}=\frac{1}{4 \pi} \int_{K / 2}^{K} \int_{0}^{b} f_{1}(a) \begin{gathered}
\operatorname{cn} b \operatorname{dn} b \\
\operatorname{sn}^{2} b
\end{gathered} \tan ^{-1} \begin{gathered}
1 \\
f_{1}(a, b)
\end{gathered} d a d b,
$$$$
I_{3}=\frac{E^{*}-k^{2} K^{*}}{2 \pi} \int_{0}^{K / 2} \int_{a}^{\kappa / 2} f_{1}(a)\left[f_{1}(a, b) \tan ^{-1} f_{4}(a, b)\right.
$$$$
\left.+\frac{1}{2} \log \left\{1+f_{t}(a, b)^{2}\right\}\right]_{\mathrm{dn} b-k^{*}}^{k^{*} \operatorname{sn} b} d b d a,
$$

$$
I_{1}=\frac{1}{2 \pi} \int_{0}^{K / 2} f_{1}(a) f_{51}(a) d a,
$$

with

$$
f_{1}(a)=i d Q_{1}
$$

given by (9.20), and with

$$
\begin{aligned}
f_{: 2}(a, b)=2 f_{\mathrm{v}}(a) & {\left[\left(\begin{array}{l}
E^{*}-k^{*} \\
K^{*}
\end{array}\right)\left(K^{*}-b\right)-\mathrm{zn}^{*} b\right], } \\
f_{3}(a, b)=f_{\mathrm{v}}(a)^{2}+\left[\left(\begin{array}{l}
E^{*} \\
K^{*}-k^{2}
\end{array}\right)\right. & \left.\left(2 K^{*}-b\right)-\mathrm{zn}^{*} b+k^{* 2} \mathrm{sn}^{*} b\right] \\
& \times\left[\mathrm{zn}^{*} b+\left(\frac{E^{*}}{K^{*}}-k^{*}\right) b+k^{* 2} \mathrm{sn}^{*} b\right],
\end{aligned}
$$

(9.36) $\quad f_{\mathrm{\imath}}(a, b)=\frac{1}{2\left(E^{*}-k^{2} K^{*}\right)}\left[\begin{array}{c}\operatorname{cn} a \operatorname{dn} a \\ \operatorname{sn} a\end{array}+\operatorname{zn} a-\frac{\operatorname{cn} b \operatorname{dn} b}{\operatorname{sn} b}-\operatorname{zn} b\right.$

$$
\left.+\left(\begin{array}{l}
E \\
K
\end{array}-k^{* 2}\right)(a-b)+k^{*}\left(\begin{array}{c}
\operatorname{cn} a \\
\operatorname{sn} a
\end{array}+\begin{array}{l}
\operatorname{cn} b \\
\operatorname{sn} b
\end{array}\right)\right],
$$

(9.37) $\quad f_{5}(a)=\left(E^{*}-k^{*} K^{*}\right)\left[\begin{array}{c}\operatorname{dn} k / 2+k^{*} \\ k^{2} \operatorname{cn} K / 2\end{array}\left\{f_{1}(a, K / 2) \tan ^{-1} \begin{array}{c}1 \\ f_{4}(a, K / 2)\end{array}\right.\right.$

$$
\begin{aligned}
&\left.+\frac{1}{2} \log \left(1+f_{1}(a, K / 2)^{2}\right)\right\}-\begin{array}{l}
\operatorname{dn} a+k^{*} \\
k^{2} \operatorname{cn} a
\end{array}\left\{f_{4}(a, a) \tan ^{-1} \frac{1}{f_{4}(a, a)}\right. \\
&\left.\left.+\frac{1}{2} \log \left(1+f_{4}(a, a)^{2}\right)\right\}\right]
\end{aligned}
$$




$$
f_{6}(a)=\frac{\operatorname{cn} a \operatorname{dn} a}{\operatorname{sn} a}+\operatorname{zn} a+\left(\frac{E}{K}-k^{* 2}\right)(a-K)+k^{*} \frac{\operatorname{cn} a}{\operatorname{sn} a} .
$$

The quantities $d(+\infty), d(0)$ and $d^{\prime}(0)$ can be computed from (9.3), (9.9) and (9.28) for any value of the parameter $k$ in the interval $0<k$ $<1$. The different values of $k$ correspond to finite cavities of different shapes, and it would be interesting to calculate several of these. However, we shall restrict ourselves here to one single example, chosen so that the cavitation parameter $\sigma$ lies in a physically significant range, while at the same time the numerical work is not excessively involved. Thus we take $k=.96$ from this stage onward.

The integrals (9.28) were computed by Simpson's rule on an IBM calculator. For numerical evaluation, the elliptic functions were expressed in terms of theta-functions. The intervals of integration were subdivided into 12 equal parts, and the results turned out to be

$$
\begin{array}{ll}
I_{1}=.002667, & I_{2}=.0152214, \\
I_{3}=.0156017, & I_{1}=.023300 .
\end{array}
$$

Therefore $d^{\prime}(0)$ has the numerical value

$$
d^{\prime}(0)=-.025587 \text {. }
$$

From this calculation and from (9.3), (9.6) and (9.9) we find

$$
\frac{d(+\infty)}{Y(+\infty)}=1, \quad \frac{d(0)}{Y(0)}=2.4338840, \quad \frac{d^{\prime}(0)}{Y(0)}=-.17471
$$

Also, by (9.10)

$$
\frac{h(0)}{Y(0)}=6.2263286, \quad \begin{aligned}
& h(0) \\
& \quad d(0)
\end{aligned}=2.5581863 .
$$

In order to evaluate the desired ratio $d(1) / Y(1)$ of the radius $d(1)$ of the three-dimensional cavity to the radius $Y(1)$ of the circular disk $\Gamma_{1}$ forming the obstacle, we interpolate by means of a quadratic polynomial in $\delta=\varepsilon /(\varepsilon+2)$, as usual. One checks easily that the expression

$$
\frac{d}{Y}=2.433884-.34942 \delta-1.084464 \delta^{2}
$$

fits the data (9.42). Putting $\delta=1 / 3$ in (9.44), we derive the final result

$$
d(1) / Y(1)=2.19691 \cong 2.197 \text {. }
$$

It is of interest to compare the answer (9.45) with the asymtotic formulas (6.3), (6.24) and (6.32) before proceeding to carry through 
more accurate and more intricate calculations. Actually, we look one step further back to the inequalities which led up to these asymptotic formulas. By (6.12) we have $Y^{2} C_{D} \leqq \sigma d^{2}$, and combining this with (8.35) we find $.827 Y^{2}(1+\sigma) \leqq \sigma d^{2}$. In the opposite direction we can use the inequality (6.18), and therefore we obtain the estimates

$$
\frac{.827 Y^{2}}{d^{2}-.827 Y^{2}} \leqq \sigma \leqq \frac{d^{2}}{h^{2}} \log \frac{h^{2}}{d^{2}}
$$

for the three-dimensional cavitation parameter $\sigma$, where a moderate error might be expected due to neglect of higher order terms in the derivation. Recalling that $h$ and $Y$ have been held fixed, we substitute the numerical data (9.43), (9.45) into (9.46) to find

$$
.2067 \leqq \sigma \leqq .2594 \text {. }
$$

The result (9.47) serves to indicate the accuracy of the asymptotic formulas (6.3), (6.24) and (6.32), but the interval in which it predicts that $\sigma$ will lie is so large that a more precise calculation is obviously called for.

10. Iterative method of solution. In this section we develop a scheme of successive approximations for determining an arbitrary threedimensional axially symmetric free surface flow and we apply the procedure to improve the accuracy in the calculation of the Riabouchinsky finite cavity begun in $\S 9$. While our iterative scheme differs in principle from the perturbation method described in $\S 3$, both approaches are based on the same type of linear mixed boundary value problem. The iterative method applies to curved obstacles and to plane flows, although such examples will not be treated here.

We explain our technique by continuing the calculation of the Riabouchinsky model introduced in $\S 9$. Let $\psi$ denote the stream function for the exact solution of the problem set there, and let $\Gamma_{2}^{*}$ denote a curve joining the point of separation $z_{0}$ to the point of reattachment $z_{0}^{*}$ and approximating the exact free boundary $\Gamma_{2}$. Neglecting terms of order higher than the first in the normal displacement $\delta n$ of $\Gamma_{2}$ into $\Gamma_{2}^{*}$, we seek a boundary value problem determining $\psi$ in the approximate flow region $\Omega^{*}$ bounded by $\Gamma_{2}^{*}$, by the fixed boundary components $\Gamma_{1}$ and $\Gamma_{1}^{*}$, and by the $x$-axis.

Clearly, $\psi$ satisfies the partial differential equation

$$
\Delta \psi-\frac{1}{y} \psi_{y}=0
$$

in $\Omega^{*}$ and satisfies the boundary condition 


$$
\psi=0
$$

on $\Gamma_{1}$ and $\Gamma_{1}^{*}$ and on the $x$-axis. The essential step is to find the boundary condition to be imposed on $\psi$ along the approximate free boundary $\Gamma_{2}^{*}$. On $\Gamma_{2}$ we have evidently

$$
\psi=0, \quad \frac{1}{y} \frac{\partial \psi}{\partial n}=1,
$$

and also from the condition that the flow should be irrotational we obtain

$$
\frac{\partial}{\partial n} \frac{1}{y} \frac{\partial \psi}{\partial n}+\frac{\kappa}{y} \frac{\partial \psi}{\partial n}=\frac{\partial q}{\partial n}+\kappa q=0
$$

there, where $q$ denotes the speed of the flow and where $\kappa$ denotes the curvature of the boundary. From (10.3) and (10.4) we derive along $\Gamma_{2}$ the relations

$$
\begin{gathered}
\frac{1}{y} \frac{\partial \psi}{\partial n}+\frac{\kappa}{y} \psi=1, \\
\frac{\partial}{\partial n}\left(\frac{1}{y} \frac{\partial \psi^{\prime}}{\partial n}+\frac{\kappa}{y} \psi\right)=\frac{\partial}{\partial n} \frac{1}{y} \frac{\partial \psi}{\partial n}+\frac{\kappa}{y} \frac{\partial \psi}{\partial n}+\psi \frac{\partial}{\partial n} \frac{\kappa}{y}=0 .
\end{gathered}
$$

It follows that the boundary condition

$$
\frac{1}{y} \frac{\partial \psi}{\partial n}+\frac{\kappa}{y} \psi=1
$$

is fulfilled along $\Gamma_{2}^{*}$ except for an error term of the order of the square $(\delta n)^{2}$ of the normal displacement $\delta n$. We impose the requirement (10.7) on $\psi$ along $\Gamma_{2}^{*}$ with the aim in mind of calculating an approximation for $\psi$ with an error of the order of magnitude $(\delta n)^{2}$.

The conditions (10.1), (10.2) and (10.7) do not quite suffice to determine $\psi$ in $\Omega^{*}$, since the value of $y^{-2} \psi$ at infinity involves the cavitation parameter $\sigma$, which is an unknown in our formulation of the problem. To compensate for this, we can use the hypothesis in our treatment that the points of separation and reattachment, $z_{0}$ and $z_{0}^{*}$, are held fixed. We showed at the end of $\S 4$ that in the neighborhood of $z_{0}$ the stream function $\psi$ has a regular power series development in terms of the variable $\left(z-z_{0}\right)^{1 / 2}$ and its conjugate. We must seek the solution of the boundary value problem (10.1), (10.2), (10.7) in a form exhibiting such a development, and in general we would expect that the answer would have a non-trivial term of the first order in $\left(z-z_{0}\right)^{1 / 2}$. However, since the velocity of the flow $\psi$ remains bounded, the first term in its 
expansion must drop out and we must have

$$
\lim _{z \rightarrow z_{0}} \frac{\psi}{\left(z-z_{0}\right)^{1 / 2}}=0, \quad \lim _{z \rightarrow z_{0}^{*}} \frac{\psi}{\left(z-z_{0}^{*}\right)^{1 / 2}}=0,
$$

where the second equation is valid at $z_{0}^{*}$ by reasons of symmetry. The addition to the original boundary value problem (10.1), (10.2), (10.7) of these conditions, which state that in a certain sense $\psi$ has critical points at $z_{0}$ and at $z_{0}^{*}$, serves to determine $\psi$ uniquely, as is shown in essence by the discussions of $\S 5$. We emphasize that the value of $y^{-2} \psi$ at infinity must be adjusted so that the relations (10.8) will hold and that it is thus necessary to allow $\psi$ to have a suitable pole at infinity in order to ensure that it have critical points at $z_{0}$ and $z_{0}^{*}$.

The boundary value problem (10.1), (10.2), (10.7), (10.8) defines an approximate solution $\psi$ of the Riabouchinsky flow problem with an error of the order of magnitude $(\delta n)^{2}$, whereas our original approximation $\Gamma_{2}^{*}$ of the free boundary $\Gamma_{2}$ was in error by precisely the amount $\delta n$. The curve $\psi=0$ defines a new approximation to $\Gamma_{2}$ and we can repeat our construction on the basis of this improved choice for $\Gamma_{2}^{*}$. The iteration process thus defined can be carried through in successive stages, each based on the previous one, until any desired degree of accuracy is achieved. The scheme is analogous to Newton's method, and the simplicity of its formulation is the only aspect in which it is distinguished from the usual approach to non-linear problems through linearization.

In connection with our derivation of the condition (10.8) to be imposed at the separation point $z_{0}$, it is worth while to insert at this stage a remark about the proper modifications to be introduced if we want to apply the iterative method to curved obstacles with the requirement that the separation be "en proue," or, in other words, that the curvature at $z_{0}$ be finite. To obtain such smooth separation, one must allow the location of $z_{0}$ to vary, and in our formulation of the problem we should compensate for this added degree of freedom by asking not only that the coefficient of $\left(z-z_{0}\right)^{1 / 2}$ vanish in the expansion of $\psi$ about $z_{0}$, as described by (10.8), but also that the coefficient of $\left(z-z_{0}\right)^{3 / 2}$ be zero. This remark settles quite simply the worst difficulty encountered in extending our procedures to include the determination of flows past more general bodies, such as the sphere.

We turn to the explicit solution of the mixed boundary value problem (10.1), (10.2), (10.7), (10.8) in the case of the Riabouchinsky model. Our first approximation $\Gamma_{2}^{*}$ to the free boundary $\Gamma_{2}$ will be based on the calculation (9.45) of the cavity altitude $d$, and because the iterative method converges so rapidly, we should find it necessary to carry through only one iteration in order to obtain quite accurate 
results.

For our choice of $\Gamma_{2}^{*}$ we perform an affine transformation on the plane free boundary given by (9.4) in order to bring it into a curve passing through the points $(-h(1), Y(1)),(0, d(1))$ and $(h(1), Y(1))$. The curve $\Gamma_{2}^{*}$ obtained in this manner has the parametric representation

$$
\begin{gathered}
x=\frac{\operatorname{zn} u}{k}+\frac{u}{k}\left(\frac{E}{K}-k^{*^{2}}\right), \\
y=\frac{1}{k}\left(k^{*^{2}}+E^{*}-k^{2} K^{*}\right)+\beta\left(\operatorname{dn} u-k^{*}\right),
\end{gathered}
$$

with $u$ in the interval $-K \leqq u \leqq K$, where $\beta$ is a constant selected so that $y=d(1)$ when $x=0$. We introduce the explicit values of the complete elliptic integrals corresponding to $k=.96$ and we use (9.45) to bring (10.9) and (10.10) into the numerical form

$$
\begin{aligned}
& x=1.0416667 \text { zn } u+.33859295 u, \\
& y=.07828039+.24348204 \operatorname{dn} u .
\end{aligned}
$$

With this definition of $\Gamma_{2}^{*}$, we shall solve the boundary value problem (10.1), (10.2), (10.7), (10.8) numerically by expressing $\psi$ as a linear combination of ten appropriate particular solutions of (10.1) and (10.8) with coefficients determined so that at 24 suitably specified points the boundary conditions (10.2) and (10.7) are fulfilled as closely as possible in the sense of least squares.

We choose as our particular solutions of (10.1) those found in Lamb [8] by separation of variables in elliptic coordinates and those obtained by placing sources and sinks along the axis of symmetry. The former system of solutions is based on ellipsoidal harmonics defined in the exteriors of the circular disks $\Gamma_{1}$ and $\Gamma_{1}^{*}$, which can be thought of as degenerate ellipsoids. The aim in introducing solutions derived by separating variables in the exteriors of the disks $\Gamma_{1}$ and $\Gamma_{1}^{*}$ is to obtain terms in our expression for the stream function $\psi$ which have at the point of separation $z_{0}$ the required development in powers of the variable $\left(z-z_{0}\right)^{1 / 2}$ and its conjugate. It is fortunate that we are able to arrive in this simple manner at a representation for $\psi$ having precisely the correct asymptotic behavior at the separation and reattachment points, so that no exceptional error is to be expected in our calculations due to the singularities of the flow at these points. As to the introduction of sources and sinks along the axis of symmetry, this technique is familiar in the construction of flows past long narrow bodies of revolution [8], and it requires no further comment.

In order to define the ellipsoidal harmonics associated with the 
disks $\Gamma_{1}$ and $\Gamma_{1}^{*}$, we need the two pairs of elliptic coordinates $\mu_{1}, \nu_{1}$ and $\mu_{2}, \nu_{2}$ given by

$$
x+(-1)^{m} h=(-1)^{m} Y \mu_{m} \nu_{m}, \quad y=Y\left(\mu_{m}^{2}+1\right)^{1 / 2}\left(1-\nu_{m}^{2}\right)^{1 / 2}
$$

with $m=1$ and $m=2$, respectively, or, in numerical form, by

$$
\left\{\begin{array}{l}
x=.91187922(-1)^{m+1}+.14645536(-1)^{m} \mu_{m} \nu_{m}, \\
y=.14645536\left(\mu_{m}^{2}+1\right)^{1 / 2}\left(1-\nu_{m}^{2}\right)^{1 / 2} .
\end{array}\right.
$$

In terms of these variables, we shall be interested in the four solutions

$$
\begin{array}{r}
p_{j}=y^{2} \sum_{m=1}^{2}\left[P_{2 j-1}^{\prime}\left(\nu_{m}\right) q_{2 j-1}^{\prime}\left(\mu_{m}\right)+(-1)^{j} j(2 j-1) q_{1}^{\prime}\left(\mu_{m}\right)\right], \\
j=2,3,4,5,
\end{array}
$$

of (10.1) and (10.8), where the functions $P_{j}^{\prime}(\nu)$ are the derivatives of the Legendre polynomials, defined by

$$
\begin{gathered}
P_{1}^{\prime}(\nu)=1, \quad P_{2}^{\prime}(\nu)=3 \nu, \\
j P_{j+1}^{\prime}(\nu)-(2 j+1) \nu P_{j}^{\prime}(\nu)+(j+1) P_{j-1}^{\prime}(\nu)=0,
\end{gathered}
$$

and where the $q_{j}^{\prime}(\mu)$ are the derivatives of the Legendre functions of the second kind along the imaginary axis defined, to be precise, by

$$
\begin{gathered}
q_{1}^{\prime}(\mu)=\frac{\mu}{\mu^{2}+1}-\cot ^{-1} \mu, \quad q_{2}^{\prime}(\mu)=3 \mu \cot ^{-1} \mu-\frac{3 \mu^{2}+2}{\mu^{2}+1}, \\
j q_{j+1}^{\prime}(\mu)+(2 j+1) \mu q_{j}^{\prime}(\mu)-(j+1) q_{j-1}^{\prime}(\mu)=0 .
\end{gathered}
$$

For large values of $\mu$ it is more convenient to use the representation [12]

$$
\begin{aligned}
(10.20) q_{j}^{\prime}(\mu)=-\frac{2^{j+2}(j+1) !}{1 \cdot 3 \cdots(2 j+1)}[\mu & \left.+\left(\mu^{2}+1\right)^{1 / 2}\right]^{-j+1}\left[\frac{\left(\mu^{2}+1\right)^{1 / 2}-\mu}{2\left(\mu^{2}+1\right)^{1 / 2}}\right]^{3 / 2} \\
& \times F\left[\frac{3}{2},-\frac{1}{2} ; j+\frac{3}{2} ; \frac{\left(\mu^{2}+1\right)^{1 / 2}-\mu}{2\left(\mu^{2}+1\right)^{1 / 2}}\right]
\end{aligned}
$$

for $q_{j}^{\prime}(\mu)$ in terms of the hypergeometric series

$$
\begin{aligned}
& F\left[\frac{3}{2},-\frac{1}{2} ; j+\frac{3}{2} ; W\right] \\
& \quad=\frac{\Gamma(j+3 / 2)}{\Gamma(3 / 2) \Gamma(-1 / 2)} \sum_{m=0}^{\infty} \frac{\Gamma(m+3 / 2) \Gamma(m-1 / 2)}{\Gamma(m+j+3 / 2) \Gamma(m+1)} W^{m} .
\end{aligned}
$$

We point out that the solutions $p_{2}, p_{3}, p_{4}, p_{5}$ of (10.1) are even in their dependence on $x$, in conformity with the symmetric formulation of our 
flow problem. We also remark that we have included in (10.15) only terms based on functions $P_{j}^{\prime}$ and $q_{j}^{\prime}$ with odd subscripts $j$, since the analogous solutions of (10.1) corresponding to even subscripts drop out of the present problem because on the disk $\Gamma_{1}$, where $\psi$ vanishes, they involve nontrivial terms of odd degree in the variable $\left(z-z_{0}\right)^{1 / 2}$. However, for more general obstacles, such as the cone or the sphere, these terms should not be omitted.

We introduce the solution

$$
p(x, y ; \xi)=\frac{\xi+x}{\left[(\xi+x)^{2}+y^{2}\right]^{1 / 2}}+\frac{\xi-x}{\left[(\xi-x)^{2}+y^{2}\right]^{1 / 2}}
$$

of (10.1), which represents the flow due to a source at the point $x=\xi$ and a sink at the point $x=-\xi$ on the axis of symmetry. In addition to $p_{2}, p_{3}, p_{4}, p_{5}$, we shall use the following six solutions of (10.1) and (10.8):

$$
\begin{gathered}
p_{1}=y^{2}, \\
p_{\jmath}=p(x, y ;[.15+.075 j] h), \quad j=6, \cdots, 10,
\end{gathered}
$$

where, of course, $h=.91187922$ represents half the distance between the disks $\Gamma_{1}$ and $\Gamma_{1}^{*}$. Our choice of the location of the sources and sinks generating the flows $p_{6}, \cdots, p_{10}$ was based on a certain amount of numerical experimentation with linear combinations of functions of the type (10.22) depending on the parameter $\xi$.

We approximate the stream function $\psi$ by an expression of the form

$$
\psi=\sum_{j=1}^{10} \lambda_{j} p_{j},
$$

where the ten coefficients $\lambda_{j}$ are to be found from the requirement that, in the sense of least squares, the boundary condition (10.2) is to be fulfilled at 11 specified points on $\Gamma_{1}$ and that, in the sense of least squares, the boundary condition (10.7) is to be fulfilled at 13 specified points along the curve $\Gamma_{2}^{*}$ defined by the parametric equations (10.11) and (10.12). An essential feature of our program will be that we impose both boundary conditions at the separation point. Thus we ask that $\psi=0$ and that $\partial \psi / y \partial n=1$ there, although these two relations are theoretically equivalent, according to (10.8). In practice it turns out that requiring $\partial \psi / y \partial n=1$ at the separation point is the most effective way to formulate (10.8) numerically, and omission of this apparently redundant condition leads to rather inaccurate results.

The points $z_{1}, \cdots, z_{12}$ on $I_{2}^{*}$ at which we impose the boundary condition (10.7) will be chosen to correspond, respectively, to the values 
$u=(l-1) K / 12$ of the parameter $u$ with $l=1, \cdots, 12$. The points $z_{14}, \cdots, z_{24}$ on $\Gamma_{1}$ at which we impose equation $(10.2)$ will be taken to have the coordinates $x=h$ and $y=\left(1-l^{2} / 100\right) Y$ with $l=0,1, \cdots, 10$, respectively, where, of course, $Y=.14645536$ is the radius of the disk $\Gamma_{1}$. We let $z_{13}=z_{14}$ and use this duplicate notation for the point of separation because we impose there the additional boundary condition $\partial \psi / y \partial n=1$. Also, at the point $z_{24}$ we must interpret the requirement (10.2) to mean that $\psi(h, y) / y^{2} \rightarrow 0$ as $y \rightarrow 0$.

We set

$$
\left(1+\kappa_{l} y_{l}\right) a_{l j}=\frac{1}{y_{i}} \frac{\partial p_{j}\left(x_{l}, y_{l}\right)}{\partial n}+\frac{\kappa_{l}}{y_{l}} p_{j}\left(x_{l}, y_{l}\right)
$$

for $l=1, \cdots, 12 ; j=1, \cdots, 10$, where $\kappa_{l}$ stands for the curvature of $\Gamma_{2}^{*}$ at the point $z_{l}=x_{l}+i y_{l}$, we set

$$
a_{l j}=\frac{1}{y_{l}} \frac{\partial p_{j}\left(x_{l}, y_{l}\right)}{\partial n}
$$

for $l=13 ; j=1, \cdots, 10$, and we set

$$
a_{l j}=\frac{1}{y_{l}^{2}} p_{j}\left(x_{l}, y_{l}\right)
$$

for $l=14, \cdots, 24 ; j=1, \cdots, 10$. We introduce the notation

(10.29) $\quad b_{l}=\frac{1}{1+\kappa_{l} y_{l}}$ for $1 \leqq l \leqq 12 ; b_{13}=1 ; b_{l}=0$ for $14 \leqq l \leqq 24$,

so that, for the purpose of our numerical calculation, the boundary conditions (10.2) and (10.7) reduce to the extremal problem

$$
\sum_{l=1}^{24}\left(\sum_{j=1}^{10} a_{l j} \lambda_{j}-b_{l}\right)^{2}=\text { minimum }
$$

for the determination of $\lambda_{1}, \cdots, \lambda_{10}$. The derivatives of the left-hand side of (10.30) with respect to the unknowns $\lambda_{m}$ must vanish at the minimum point, whence we derive the system

$$
\sum_{j=1}^{10}\left(\sum_{l=1}^{24} a_{l m} l_{j}\right) \lambda_{j}=\sum_{l=1}^{24} a_{l m} b_{l}
$$

of ten simultaneous linear equations to be solved for the ten parameters $\lambda_{m}$.

The elements of the ractangular matrix $\mathrm{a}_{l j}$ were computed on an IBM calculator, using $(10.11), \cdots,(10.22)$. The results are listed in Table I. The ten simultaneous linear equations (10.31) for the unknowns 
$\lambda_{1}, \cdots, \lambda_{10}$ were solved numerically on an IBM calculator, and the answer was substituted into (10.25) to yield for the stream function $\psi$ the approximation

$$
\begin{aligned}
\psi= & .450770 p_{1}-.0747027 p_{2}-.0136344 p_{3}-.00412697 p_{4} \\
& -.000848527 p_{5}-.0722477 p_{6}+.193757 p_{7} \\
& -.267881 p_{8}+.206857 p_{9}-.0913614 p_{10} .
\end{aligned}
$$

The error in this numerical solution of the boundary value problem (10.1), (10.2), (10.7), (10.8) should be proportional to the values of the 24 expressions

$$
e_{l}=\sum_{j=1}^{10} a_{l j} \lambda_{j}-b_{l},
$$

since

$$
\left(1+\kappa_{\imath} y_{l}\right) e_{l}=\frac{1}{y_{l}} \frac{\partial \psi\left(x_{l}, y_{l}\right)}{\partial n}+\frac{\kappa_{l}}{y_{l}} \psi\left(x_{l}, y_{l}\right)-1
$$

for $l=1, \cdots, 12$, since

$$
e_{l}=\frac{\psi\left(x_{l}, y_{l}\right)}{y_{l}^{2}}
$$

for $l=14, \cdots, 24$, and since $y_{13} e_{13}=\partial \psi\left(x_{13}, y_{13}\right) / \partial n-y_{13}$. The numerical values of the quantities $e_{l}$ are listed in Table II and they indicate an error in $\psi$ of about 1.5 per cent, which is not surprising in view of the small number of functions used in our interpolation to the solution of the linear problem (10.1), (10.2), (10.7), (10.8).

From (10.23), (10.25) and the asymptotic expansion $\psi \sim y^{2} / 2(1+\sigma)^{1 / 2}$, we find that the cavitation parameter $\sigma$ is given by

$$
\sigma=\frac{1}{4 \lambda_{1}^{2}}-1,
$$

and therefore we derive from (10.32) the numerical estimate

$$
\sigma=.23035 \cong .23
$$

for $\sigma$. We calculate the drag coefficient $C_{D}$ directly from the definition (6.2) by numerical integration based on subdivision of the nose $\Gamma_{1}$ into the ten intervals whose end-points have the ordinates $y_{14}, y_{15}, \cdots, y_{24}$ and evaluation of $(\nabla \psi)^{2}=\psi_{x}^{2}$ at the subdivision points by means of (10.32). We obtain in this manner the result

$$
\frac{C_{D}(\sigma)}{(1+\sigma)}=.89438 \cong .89,
$$


or

$$
C_{D}(.23)=1.1
$$

The values of the pressure $p=\left[1-(\nabla \varphi)^{2}\right] / 2$ at the subdivision points on $\Gamma_{1}$ are listed in Table III.

We also determine from (10.32) a numerical estimate of the cavity shape. Since we should have $\psi=0$ and $\partial \psi / \partial n=y$ along the exact free streamline $\Gamma_{2}$, we conclude that the normal displacement $\delta n$ of $\Gamma_{2}$ into $\Gamma_{2}^{*}$ has on $\Gamma_{2}^{*}$ the value $\delta n=\psi / y$ to the first order of approximation. Thus substitution into the formula

$$
\tilde{z}=z-\frac{\psi}{y} \frac{\partial z}{\partial n}
$$

of the equations (10.11) and (10.12) for $\Gamma_{2}^{*}$ yields points $\tilde{z}$ on a higher order approximation to the exact free boundary curve $\Gamma_{2}$. In Table IV we have listed the coordinates $x_{l}, y_{l}$ of the 13 points $z_{1}, \cdots, z_{13}$ on the first approximation $\Gamma_{2}^{*}$, together with the corresponding values of the normal shift $\psi\left(x_{l}, y_{l}\right) / y_{l}$ and the coordinates $\tilde{x}_{l}, \tilde{y}_{l}$ of the corresponding points on our second approximation to the free boundary. Since $z_{1}$ lies at the widest section of the cavity, (10.40) provides in addition the new estimate

$$
\frac{d(1)}{Y(1)}=2.38
$$

for the cavity radius $d=d(1)$, indicating that the actual cavity is larger than the approximation we found by affine transformation of the plane free streamline. Notice that near the separation point numerical errors deprive $\psi / y$ of geometrical significance, although higher terms in the expansion of $\psi$ there still describe $\Gamma_{2}$.

We compare the numerical estimate (10.38) of the drag coefficient $C_{D}$ with our earlier calculation (8.34), using the asymptotic formula (6.3). We start by establishing the more precise inequality

$$
\frac{C_{D}(\sigma)}{1+\sigma} \geqq C_{D}(0)
$$

which is valid for all values of $\sigma$ and for arbitrary nose shapes, provided that the point of separation $z_{0}$ is held fixed. To prove (10.42), we show that the term on the left is an increasing function of $\sigma$. From the definition (6.2) of $C_{D}(\sigma)$, it suffices to establish that

$$
\frac{\partial^{2} \psi}{\partial \sigma \partial n} \leqq 0
$$


on the obstacle $\Gamma_{1}$. For this purpose we study the solution $\partial \psi / \partial \sigma$ of the basic equation (10.1). We suppose that the nose $\Gamma_{1}$ is held fixed, while we allow the tail $\Gamma_{1}^{k}$ to approach $\Gamma_{1}$ steadily as $\sigma$ increases. Thus $\partial \psi / \partial \sigma>0$ on $\Gamma_{1}^{*}$, whereas $\partial \psi / \partial \sigma=0$ on $\Gamma_{1}$ and along the axis of symmetry. At infinity $\partial \psi / \partial \sigma \sim-y^{2} / 4(1+\sigma)^{3 / 2}$ by (6.1), and from our perturbation technique we easily deduce that $\partial \psi / \partial \sigma$ satisfies the homogeneous boundary condition

$$
\frac{\partial}{\partial n} \frac{\partial \psi}{\partial \sigma}+\kappa \frac{\partial \psi}{\partial \sigma}=0
$$

along $\Gamma_{2}$. Since the point of separation $z_{0}$ remains fixed, we conclude that $\partial \psi / \partial \sigma$ has a critical point there. Hence a level curve $\partial \psi / \partial \sigma=0$ must emanate from $z_{0}$, and the analysis of $\$ 5$ shows that this level curve divides the flow region $\Omega$ into a connected subregion $\Omega^{+}$where $\partial \psi / \partial \sigma>0$ and a connected subregion $\Omega^{-}$where $\partial \psi / \partial \sigma<0$. The point at infinity lies on the boundary of $\Omega^{-}$, whence $\Omega^{-}$must border the entire nose $\Gamma_{1}$, and (10.43) follows. In addition, the subregion $\Omega^{+}$must border the entire free boundary $\Gamma_{2}$, and thus the cavity must shrink monotonically as $\sigma$ increases.

The monotonicity of $C_{D}(\sigma) /(1+\sigma)$ as a function of $\sigma$ and the numerical results $(8.34),(10.73)$ and $(10.38)$ show that

$$
.827(1+\sigma) \leqq C_{D}(\sigma) \leqq .89(1+\sigma)
$$

in the interval $0 \leqq \sigma \leqq .23$. It is apparent from the asymptotic formula (6.3) that the bound on the right in (10.45) is considerably too large. Consequently we must make a detailed analysis of the errors in the numerical calculations performed thus far before proceeding to draw conclusions from the data.

The oscillations in the figures listed in Table III indicate a substantial error in our determination of the pressure distribution along the disk $\Gamma_{1}$, since the pressure $p$ should be a monotonic function of $y$ there. An error of this type would appear to be unavoidable, since calculation of the pressure involves computing derivatives of the stream function $\psi$ on the boundary of the region in which the flow is defined. A crude analogy with the problem of approximating a function and its derivative by the same number of terms of a power series expansion indicates that the expression (10.32) for $\psi$ in terms of the ten solutions $p_{j}$ of (10.1) could result in errors in evaluating derivatives which are roughly ten times as large as the error in $\psi$ itself, which we estimated to be about 1.5 per cent. This difficulty has led us to an inaccurate value (10.38) for the drag coefficient, although the figures given in Table III do display some of the characteristics of the pressure distribution, which remains almost constant near the center of the disk, but 
falls off sharply at the edge.

Similarly, the normal shift $\delta n=\psi / y$ is computed at the boundary of the region of definition of $\psi$, and it also exhibits an unusually large error. However, we can assume that the sign of the normal shift is correct, and it is probably safe to conclude that the exact free boundary lies half way between our first approximation $\Gamma_{2}^{*}$ and the corresponding approximation which we would have obtained if we had neglected the sizable term involving $\delta^{2}$ in (9.44). Thus the estimate

$$
\frac{d(1)}{Y(1)}=2.26
$$

can be expected to differ as little as 1.5 per cent from the true value of $d(1) / Y(1)$.

More precise information about the errors just discussed can be found through approximation of $\psi$ by linear combinations of fewer than ten of the functions $p_{j}$. The formula

$$
\begin{aligned}
\psi= & .449026 p_{1}-.0487115 p_{2}-.00717523 p_{3}-.00278161 p_{4} \\
& -.0278822 p_{6}+.0312522 p_{8}-.0390783 p_{10}
\end{aligned}
$$

gives the best approximation in the sense of least squares to the boundary conditions (10.2) and (10.7) at the points $z_{1}, \cdots, z_{2 \pm}$ when we use only the seven solutions $p_{1}, p_{2}, p_{3}, p_{4}, p_{6}, p_{8}, p_{10}$ of (10.1). The average error in fitting the boundary conditions here is 3.3 per cent. The value obtained for $C_{D}(\sigma) /(1+\sigma)$ is .94 and the value obtained for the normal shift $\delta n=\psi / y$ at the intersection of $\Gamma_{2}^{*}$ with the $y$-axis is -.055 . These results are obviously out of line with the actual solution of the problem. On the other hand, (10.47) gives for $\sigma$ the more reasonable estimate

$$
\sigma=.23993 \text {. }
$$

Our investigation of (10.47) shows that addition of the three terms involving $p_{5}, p_{7}$, and $p_{9}$ in the approximation to the stream function $\psi$ improves the accuracy decisively. Computation of the pressure distribution on $\Gamma_{1}$ for the flow (10.47), for example, establishes that the oscillations in the pressure are significantly smoothed out when we include $p_{5}, p_{7}$, and $p_{9}$ in our interpolation scheme. We conclude that the principal errors in our numerical calculation of the free surface flow $\psi$ could be eliminated by interpolating with a larger number of solutions of (10.1) and by fitting the boundary conditions (10.2) and (10.7) at more points along $\Gamma_{1}$ and $\Gamma_{2}^{*}$. A linear combination of fifteen or twenty solutions satisfying the boundary conditions at forty or fifty points in the sense of least squares should suffice to give physically significant results.

In spite of the limited accuracy of our present numerical solution 
of the linear boundary value problem (10.1), (10.2), (10.7), (10.8), we can still deduce from it relevant information about the flow. In particular, the small difference between the two estimates (10.37) and (10.48) of $\sigma$ indicates that the equation $1+\sigma=1.23$ is in error by less than 1 per cent. The explanation for this increase in the accuracy of our determination of $\sigma$ over that encountered in evaluating the drag lies in the fact that the formula (10.36) for $\sigma$ involves only the quantity $\lambda_{1}$, which represents a coefficient in the expansion of $\psi$ about the point at infinity. Such quantities, whose calculation depends only on the characteristics of $\psi$ in the interior of the flow region, can be computed with an error significantly smaller than the error occurring in our approximation to the boundary conditions. The situation here is analogous to the rapid convergence of a power series near the center of its circle of convergence. These remarks suggest that we attempt to evaluate the drag coefficient in terms of quantities which can be calculated in the interior of the flow region, and this is indeed made possible by the variational formula (2.18) for the drag.

By introducing the coefficient $a$ in the expansion (2.1) of the velocity potential $\varphi$ about the point at infinity and making use of the familiar representation (2.6) for $a$ in terms of the virtual mass $M$, we can bring (2.18) into the more suitable form

$$
\frac{C_{D}(\sigma)}{1+\sigma}=\frac{3}{2 h Y^{2}}\left[\begin{array}{cc}
V & 4 a \\
\pi & 1+\sigma
\end{array}\right] .
$$

The chief difficulty encountered in applying (10.49) lies in the calculation of the volume $V$. For the flow (10.32) it is useless to attempt to compute $V$, since this would again lead to integrals over the boundary of the flow region and would therefore involve large errors. However, the stationary character of the right-hand side of (10.49), as established in $\S 2$, spares us the necessity of using the approximation (10.32). Instead, we calculate directly the flow past the body bounded by the disks $\Gamma_{1}$ and $\Gamma_{1}^{*}$ and the approximate free boundary $\Gamma_{2}^{*}$ and use the values of $V$ and $a$ for this flow in (10.49), together with the value of $\sigma$ computed earlier. According to the variational principle (2.7), this procedure should yield an exceptionally accurate lower bound for the drag coefficient $C_{D}$. The only source of significant errors will stem from subtracting two large numbers on the right in (10.49) to determine a small number on the left.

In order to calculate the flow past $\Gamma_{1}, \Gamma_{1}^{*}$ and $\Gamma_{2}^{*}$, we write its stream function $\psi^{*}$ in the form

$$
\psi^{*}=p_{1}+\sum_{j=2}^{10} \lambda_{j}^{*} p_{j}
$$


and choose $\lambda_{2}^{*}, \cdots, \lambda_{10}^{*}$ to fit the boundary condition $\psi^{*} / y^{2}=0$ at the points $z_{1}, \cdots, z_{24}$ in the sense of least squares. Setting

$$
c_{l j}=\frac{p_{j}\left(x_{l}, y_{l}\right)}{y_{l}^{2}}
$$

for $l=1, \cdots, 24 ; j=1, \cdots, 10$, we arrive at the extremal problem

$$
\sum_{l=1}^{24}\left(\sum_{j=2}^{10} c_{l j} \lambda_{j}^{*}+1\right)^{2}=\text { minimum }
$$

for the determination of $\lambda_{2}^{*}, \cdots, \lambda_{10}^{*}$, whence, as in our previous example,

$$
\sum_{j=2}^{10}\left(\sum_{l=1}^{24} c_{l m} c_{l j}\right) \lambda_{j}^{*}=-\sum_{l=1}^{24} c_{l m} .
$$

The coefficients $c_{l j}$ can be obtained from Tables I and V, which were compiled before during our development of the flow (10.32). We use the solution of the system (10.53) of nine simultaneous linear equations to derive for $\psi^{*}$ the approximate expression

$$
\begin{aligned}
\psi^{*}=p_{1} & -.138356 p_{2}-.0270098 p_{3}-.00570592 p_{4} \\
& -.00232521 p_{5}-.139902 p_{6}+.372071 p_{7} \\
& -.507771 p_{8}+.388026 p_{9}-.169804 p_{10} .
\end{aligned}
$$

The average deviation here from the boundary condition $\psi^{*} / y^{2}=0$ is 1.2 per cent.

From (10.54) we derive for $a$ the numerical value

$$
a=.041236 \text {, }
$$

while explicit integration gives for $V$ the result

$$
V=.458433 \text {. }
$$

Substituting this data into (10.49), we arrive at the approximate relation

$$
\frac{C_{D}(\sigma)}{1+\sigma}=11.19100-\frac{12.64977}{1+\sigma},
$$

which yields for $C_{D}$ the final estimate

$$
\frac{C_{D}(\sigma)}{1+\sigma}=.90956 \cong .91
$$

when $\sigma=.23035$. This result is in quite good agreement with the 
earlier value (10.38), but is still subject to suspicion because of its sensitivity to small variations in the value of $\sigma$. For example, in order to obtain the quite possibly correct value .83 in (10.58) using (10.57), it would suffice to replace (10.37) by the reduced estimate $\sigma=.221$.

The best criterion for detecting sources of error such as we have discussed here is an examination of the pressure distribution along the obstacle $\Gamma_{1}$. Only when we have achieved accuracy sufficient to yield a properly behaved pressure distribution should we expect final acceptance of our numerical data. Thus, in contrast with the situation in $\S \S 7$ and 8, no decisive statement of conclusions can be made on the basis of the preliminary numerical data accumulated in the present section. However, the material developed here can be considered as a sufficiently successful beginning to assure us that our fundamental method of attack on the free surface flow problem will yield answers of any desired degree of accuracy when we push through a more elaborate numerical analysis of the linear boundary value problem (10.1), (10.2), (10.7), (10.8) by interpolating with a significantly larger number of solutions of (10.1). In support of this contention, we note that our numerical data all lie in a reasonable range and that the improvement in results has been satisfactory when we advanced the number of interpolating functions from seven to ten. Thus the only major trouble we have encountered occurs in solving (10.1), (10.2), (10.7), (10.8) in a given region, and apparently we have overcome completely the original difficulty of not knowing beforehand the shape of the free boundary. Only limitations on time, space and patience prevent us from carrying through a definitive calculation in this initial report. In any case, it is to be hoped that the numerical data presented here will provide an adequate description of our method for the reader interested in pursuing the problem further.

In closing, I should like to express my appreciation to Michael Maschler for an exceptionally fine job of preparing my numerical calculations for the IBM Card-Programmed Electronic Calculator at the Stanford Computation Center. I am also immeasurably indebted to Gladys Garabedian for untiring efforts on a desk calculator and to Priscilla Feigen for an elegantly typed manuscript. 


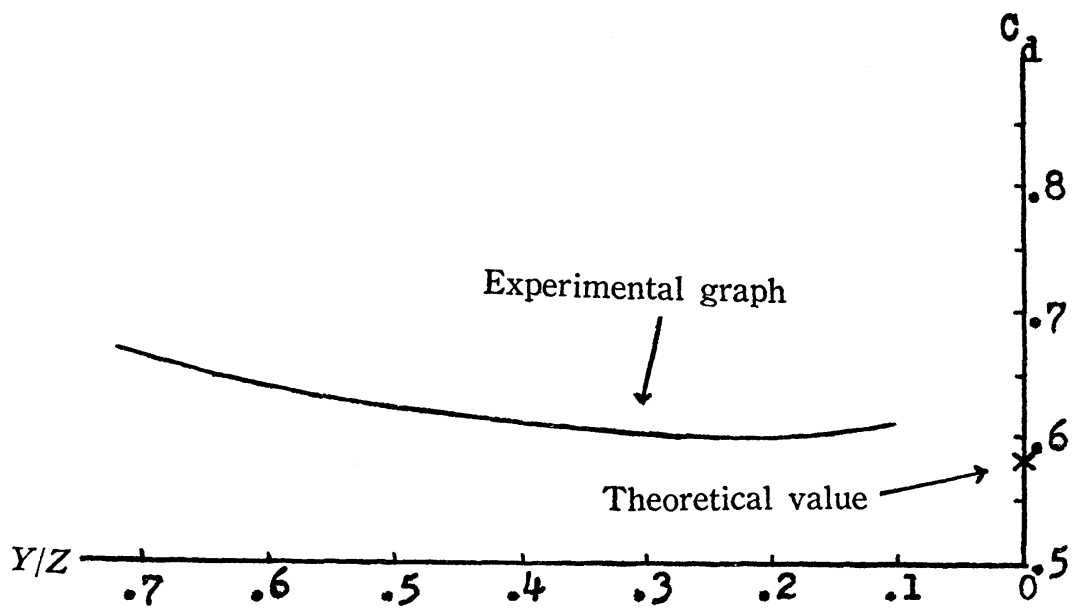

Fig. 1. Discharge coefficient vs. orifice ratio.

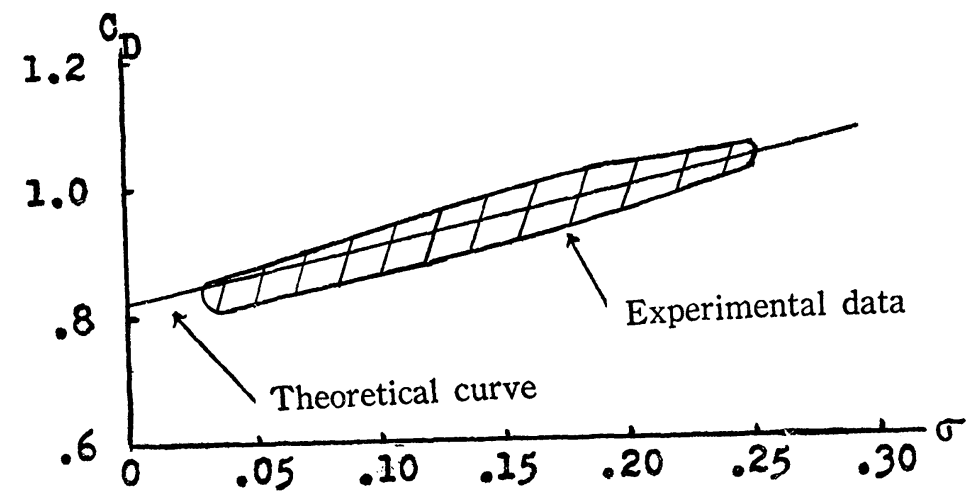

Fig. 2. Drag coefficient vs. cavitation parameter.

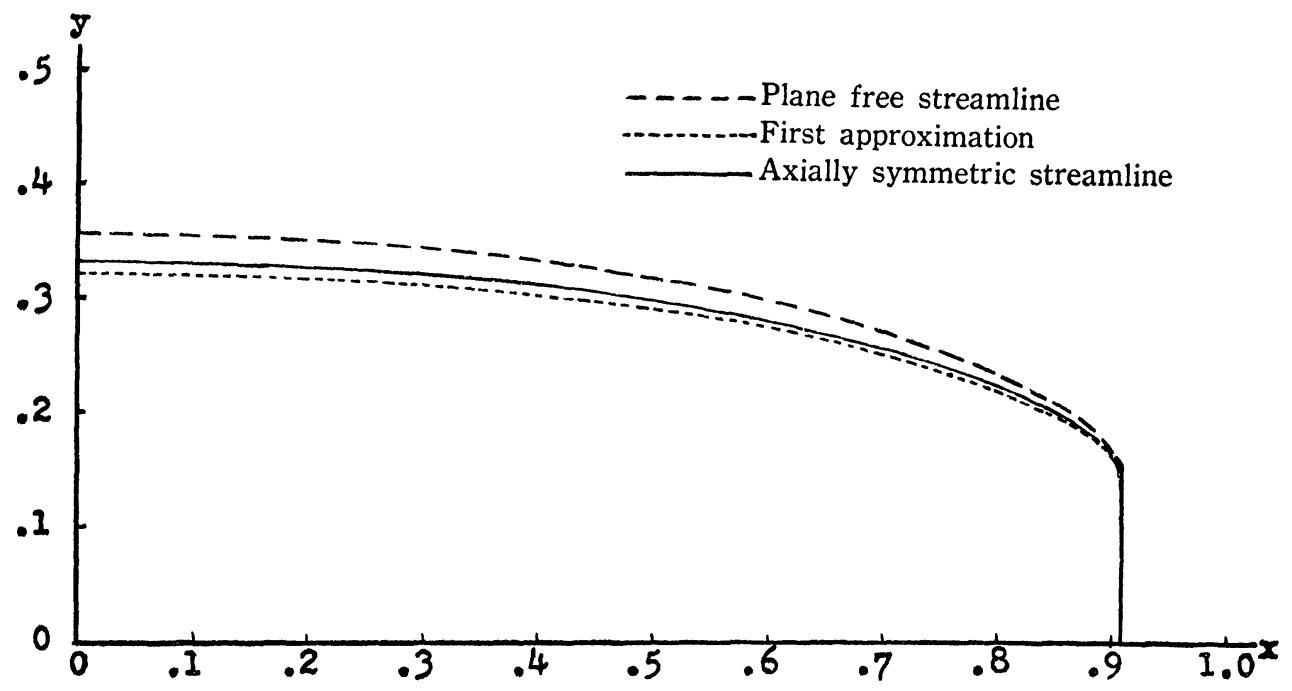

Fig. 3. Sketch of the axially symmetric cavity shape. 


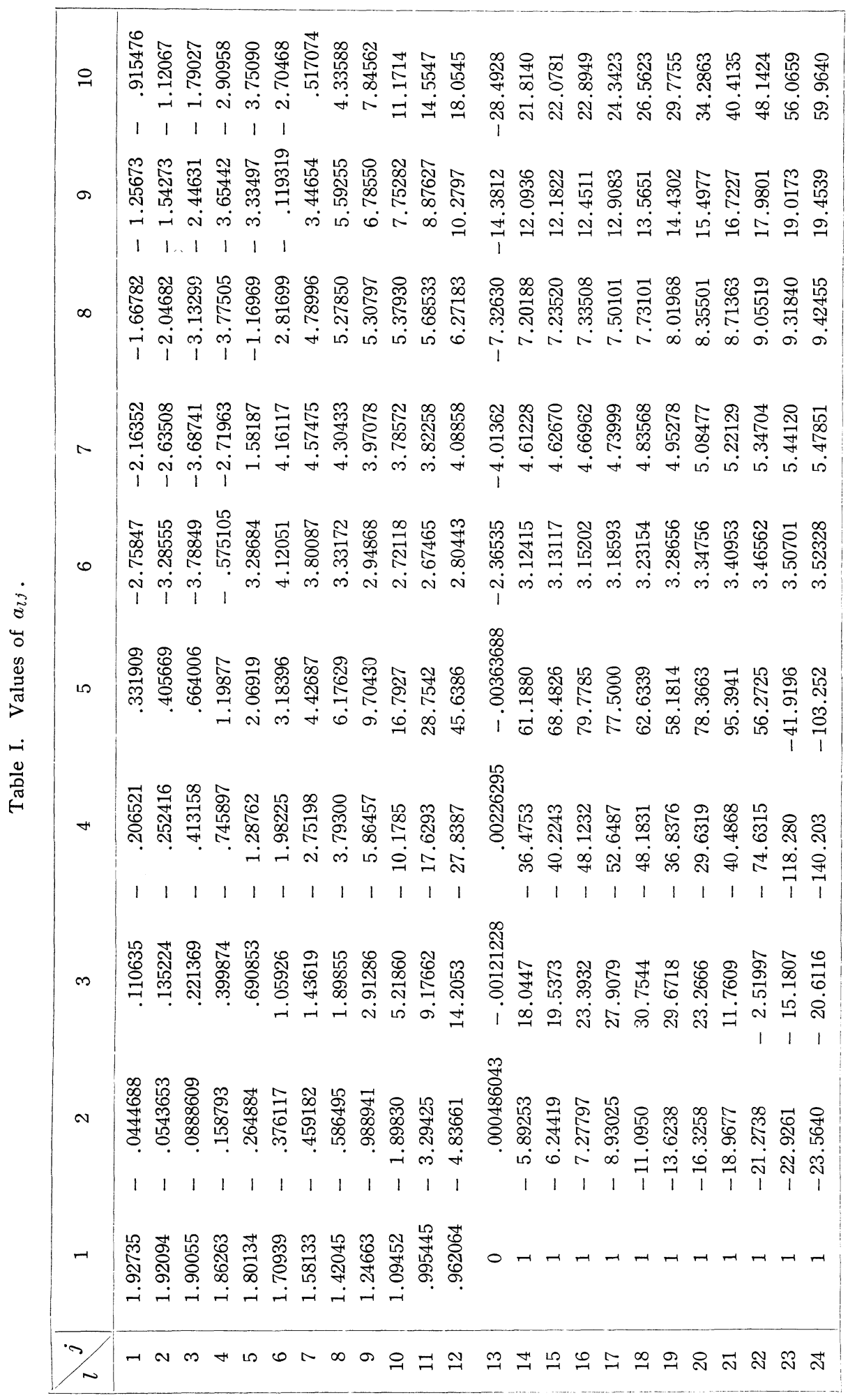




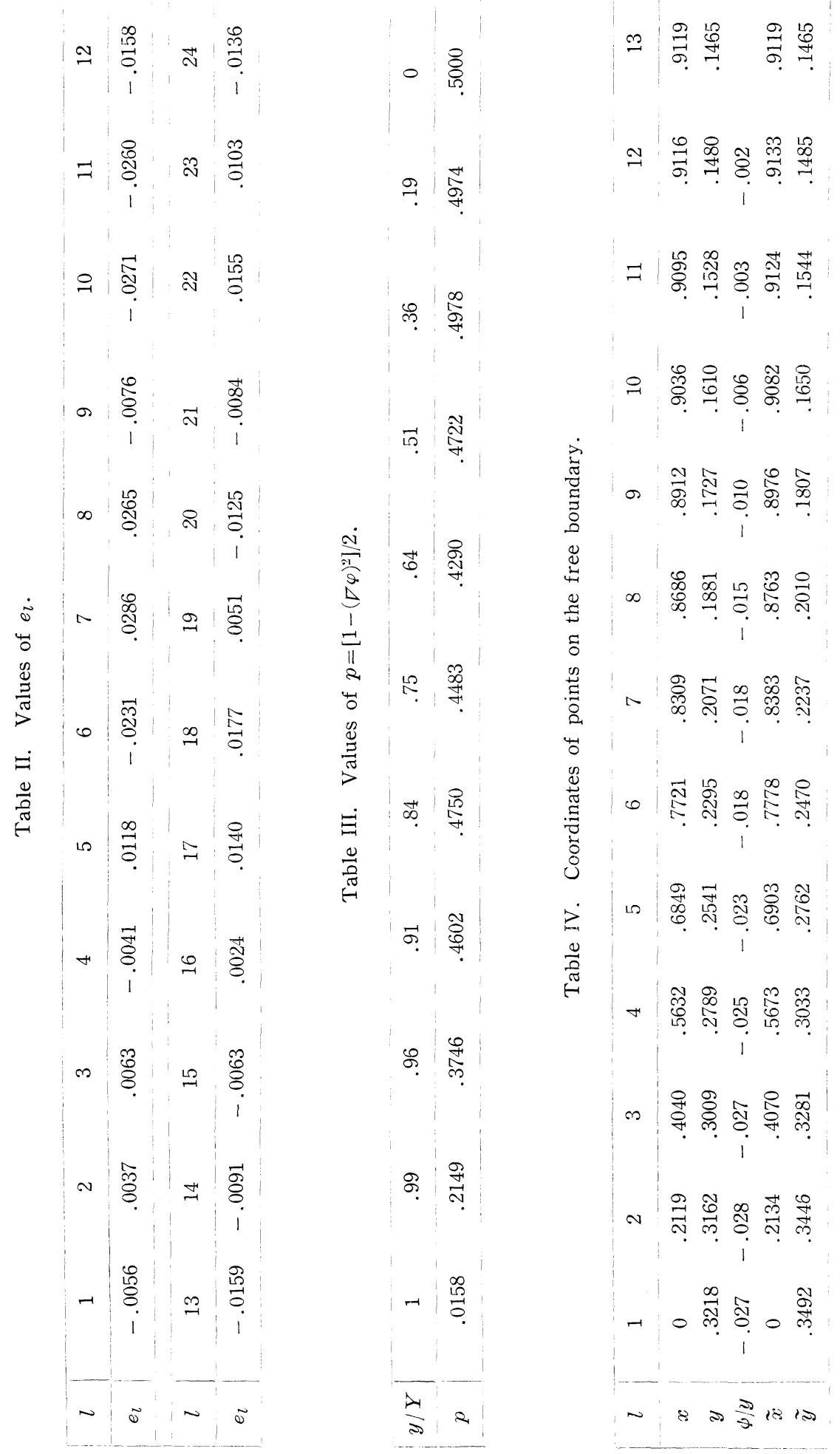


\&

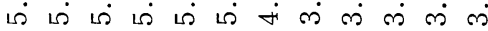

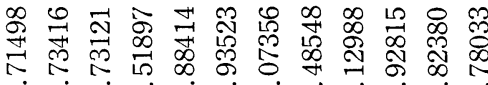

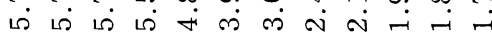

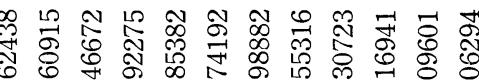
เि เि

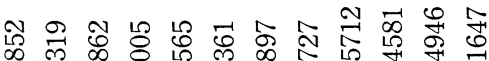

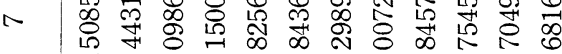

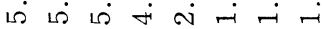

3
3
3
0
0
0
0
0
0
0

m.

웅 范

范

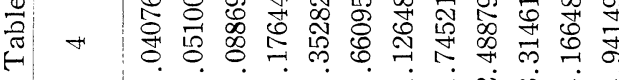
$1, i j$ तi

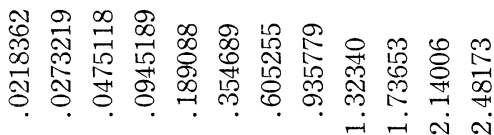

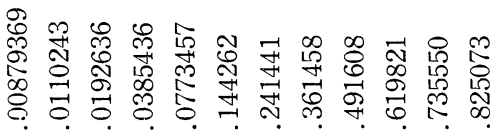

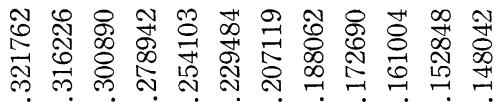

우

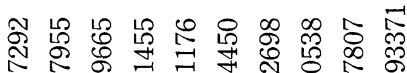

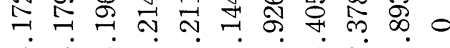

मं

त

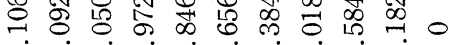

i i

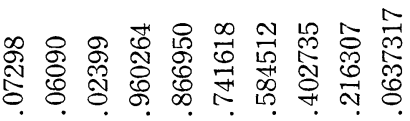

$\infty$ †े

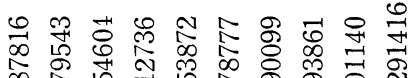

- $\quad$ เ

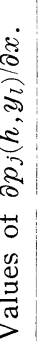

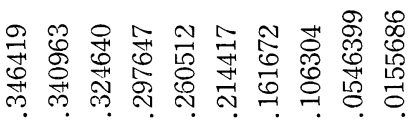

6

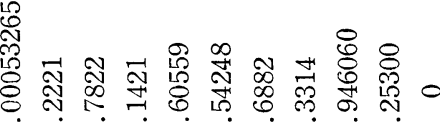

เ 1
1

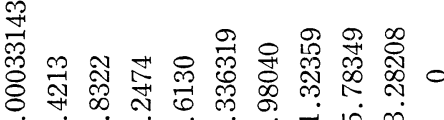

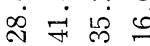

10

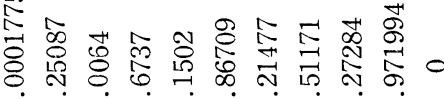

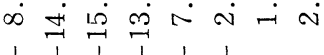

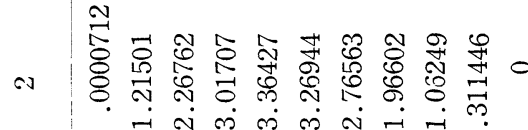

$-00000000000$

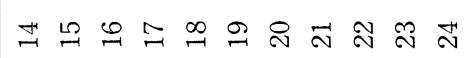




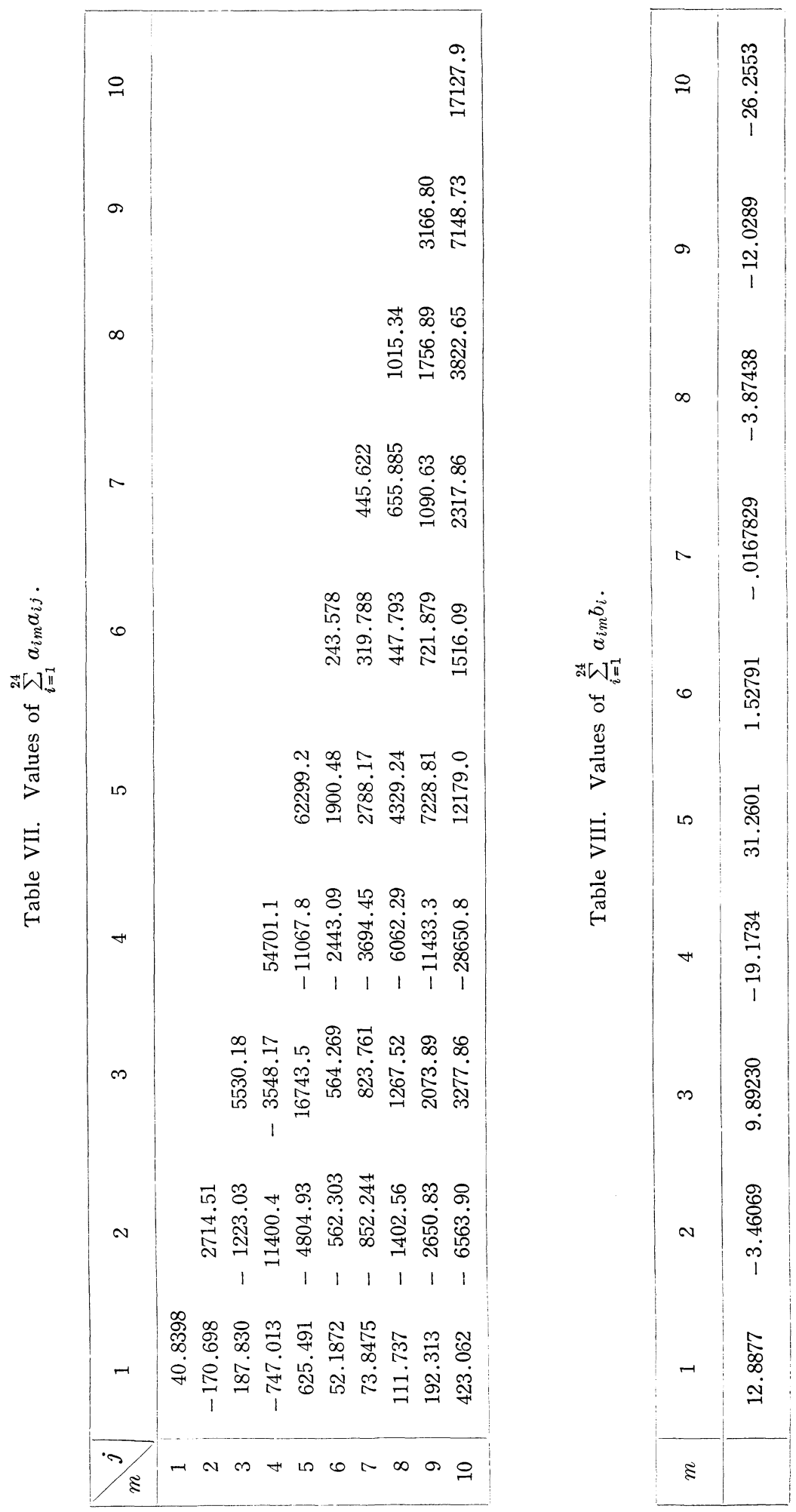



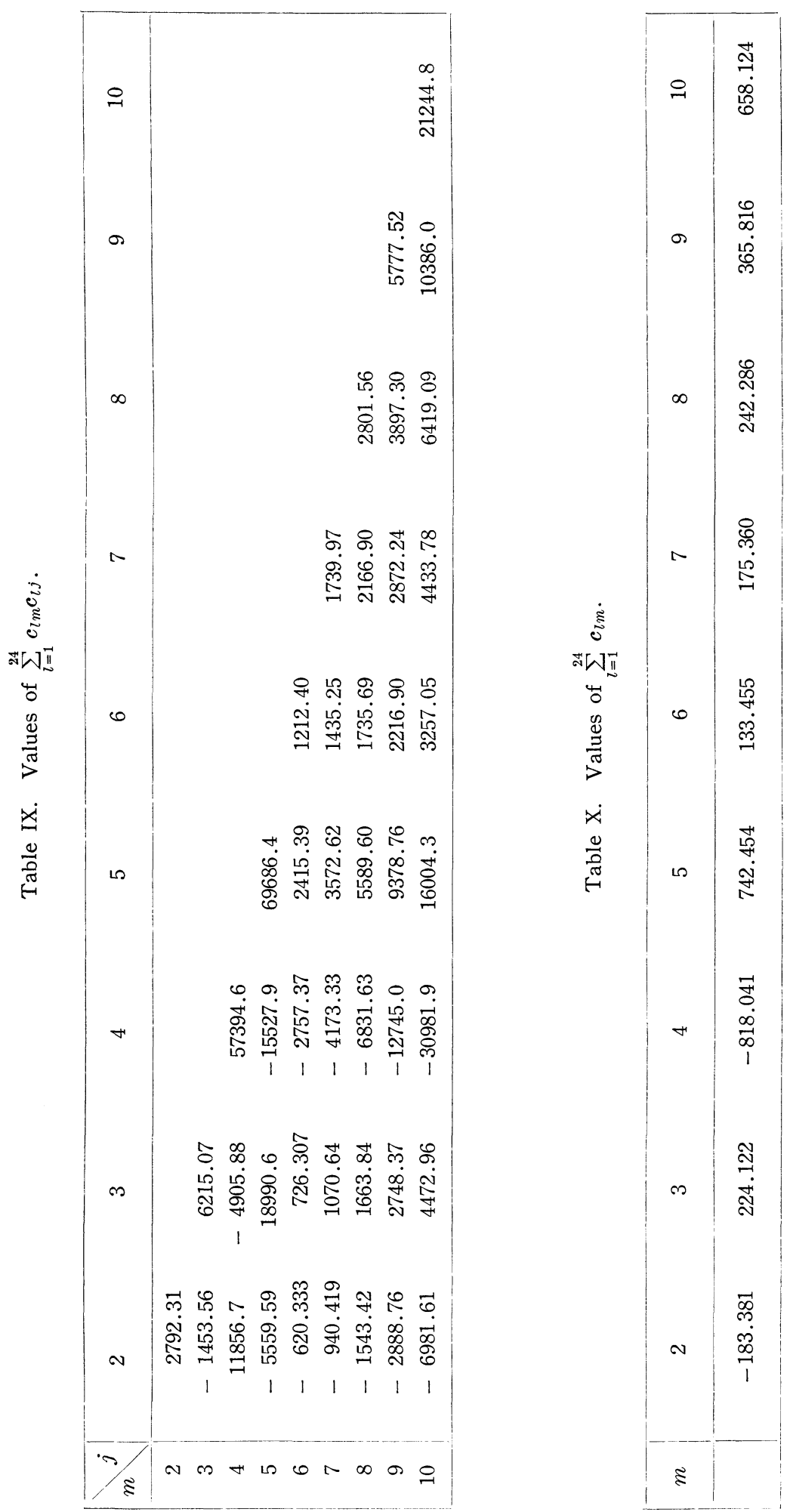


\section{REFERENCES}

1. A. H. Armstrong and J. H. Dunham, Axisymmetric cavity flow, Armament Research Establishment Report 12/53, London, 1953.

2. P. Eisenberg, On the mechanism and prevention of cavitation, David Taylor Model Basin Report 712, 1950.

3. K. Friedrichs, Über ein Minimumproblem für Potentialströmungen mit freiem Rande, Math. Ann., 109 (1933), 60-82.

4. P. R. Garabedian, An example of axially symmetric flow with a free surface, Studies in mathematics and mechanics presented to Richard von Mises, 149-159, New York, 1954.

5. P. R. Garabedian, H. Lewy and M. Schiffer, Axially symmetric cavitational flow, Ann. Math., 56 (1952), 560-602.

6. P. R. Garabedian and D. C. Spencer, Extremal methods in cavitational flow, J. Ratl. Mech. Anal., 1 (1952), 359-409.

7. D. Gilbarg, Uniqueness of axially symmetric flows with free boundaries, J. Ratl. Mech. Anal., 1 (1952), 309-320.

8. H. Lamb, Hydrodynamics, New York, 1932.

9. W. M. Lansford, Discharge coefficients for pipe orifices, Civil Engineering, 4 (1934), 245-247.

10. N. Levinson, On the asymptotic shape of the cavity bchind an axially symmetric nose moving through an ideal fluid, Ann. Math., 47 (1946), 704-730.

11. F. Oberhettinger and W. Magnus, Anwendung der elliptischen Funktionen in Physik und Technik, Berlin, 1949.

12. W. Magnus and F. Oberhettinger, Formulas and theorems for the special functions of mathematical physics, New York, 1949.

13. M. S. Plesset and B. Perry, On the application of free streamline theory to cavity flows, Mémoires sur la méchanique des fluides offerts à M. D. Riabouchinsky à l'occasion de son jubilé scientifique, Publications scientifiques et techniques du ministère de l'air, Paris, 1954.

14. H. Reichardt, The laws of cavitation bubbles at axially symmetric bodies in a flow, MAP Reports and Translations No. 766, 1946.

15. H. Rouse and A.-H. Abul-Fetouh, Characteristics of irrotational flow through axially symmetric orifices, J. Appl. Mech., 17 (1950), 1-6.

16. R. V. Southwell and G. Vaisey, Relaxation methods applied to engineering problems. XII. Fluid motions characterized by 'free' streamlines, Phil. Trans. Roy. Soc., 240 (1946), 117-161.

17. E. Trefftz, Über die Kontraktion kreisförmiger Flüssigkeitsstrahlen, Zeit. Math. Phys., 64 (1916), 34-61.

18. A. Weinstein, Les conditions aux limites introduites par l'hydrodynamique, Enseignment Math., 35 (1936), 107-125.

\section{STANFORD UNIVERSITY}




\section{PACIFIC JOURNAL OF MATHEMATICS}

\section{EDITORS}

H. L. Royden

Stanford University

Stanford, California

E. Hewits

University of Washington

Seattle 5 , Washington
R. P. Dilworth

California Institute of Technology Pasadena 4, California

E. G. Straus

University of California

Los Angeles 24, California

\section{ASSOCIATE EDITORS}
E. F. BECKENBACH
C. E. BURGESS
H. BUSEMANN
H. FEDERER

\author{
M. HALL \\ P. R. HALMOS \\ V. GANAPATHY IYER \\ R. D. JAMES
}

\author{
M. S. KNEBELMAN \\ I. NIVEN \\ T. G. OSTROM \\ M. M. SCHIFFER
}

\section{J. J. STOKER \\ G. SZEKERES \\ F. WOLF \\ K. YOSIDA}

\section{SUPPORTING INSTITUTIONS}

UNIVERSITY OF BRITISH COLUMBIA CALIFORNIA INSTITUTE OF TECHNOLOGY

UNIVERSITY OF CALIFORNIA

MONTANA STATE UNIVERSITY

UNIVERSITY OF NEVADA

OREGON STATE COLLEGE

UNIVERSITY OF OREGON

UNIVERSITY OF SOUTHERN CALIFORNIA
STANFORD UNIVERSITY

UNIVERSITY OF UTAH

WASHINGTON STATE COLLEGE

UNIVERSITY OF WASHINGTON

$*$ * * *

AMERICAN MATHEMATICAL SOCIETY

CALIFORNIA RESEARCH CORPORATION HUGHES AIRCRAFT COMPANY

Mathematical papers intended for publication in the Pacific Journal of Mathematics should be typewritten (double spaced), and the author should keep a complete copy. Manuscripts may be sent to any of the editors. Manuscripts intended for the outgoing editors should be sent to their successors. All other communications to the editors should be addressed to the managing editor, E. G. Straus at the University of California, Los Angeles 24, California.

50 reprints of each article are furnished free of charge; additional copies may be obtained at cost in multiples of 50 .

The Pacific Journal of Mathematics is published quarterly, in March, June, September, and December. The price per volume (4 numbers) is $\$ 12.00$; single issues, $\$ 3.50$. Back numbers are available. Special price to individual faculty members of supporting institutions and to individual members of the American Mathematical Society: $\$ 4.00$ per volume; single issues, $\$ 1.25$.

Subscriptions, orders for back numbers, and changes of address should be sent to Pacific Journal of Mathematics, 2120 Oxford Street, Berkeley 4, California.

Printed at Kokusai Bunken Insatsusha (International Academic Printing Co., Ltd.), No. 10, 1-chome, Fujimi-cho, Chiyoda-ku, Tokyo, Japan.

\section{PUBLISHED BY PACIFIC JOURNAL OF MATHEMATICS, A NON-PROFIT CORPORATION}

The Supporting Institutions listed above contribute to the cost of publication of this Journal, but they are not owners or publishers and have no responsibility for its content or policies. 


\section{Pacific Journal of Mathematics}

\section{Vol. 6, No. 4}

1956

Seymour Ginsburg, On mappings from the family of well ordered subsets of

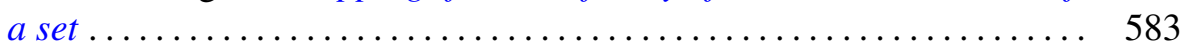

Leon Ehrenpreis, Some properties of distributions on Lie groups ......... 591

Marion K. Fort, Jr., A geometric problem of Sherman Stein ............. 607

Paul R. Garabedian, Calculation of axially symmetric cavities and jets . . . . 611

Walter Mossman Gilbert, Completely monotonic functions on cones ...... 685

William L. Hart and T. S. Motzkin, A composite Newton-Raphson gradient method for the solution of systems of equations ................. 691

C. W. Mendel and I. A. Barnett, A functional independence theorem for square matrices ................................. 709

Howard Ashley Osborn, The problem of continuous programs .......... 721

William T. Reid, Oscillation criteria for linear differential systems with complex coefficients ............................. 733

Irma Reiner, On the two-adic density of representations by quadratic

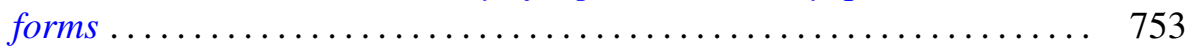

Shoichiro Sakai, A characterization of $W^{*}$-algebras .............. 763

Robert Steinberg, Note on a theorem of Hadwiger................. 775

$\mathrm{J}$. Eldon Whitesitt, Construction of the lattice of complemented ideals within

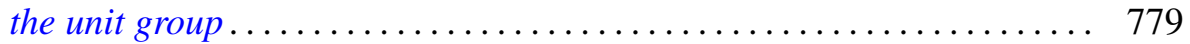

Paul Civin, Correction to "Some ergodic theorems involving two operators"... 